\title{
UNDERSTANDING WORKPLACE INTERPERSONAL MISTREATMENT IN THE NZ MILITARY: STRUCTURE, ANTECEDENTS AND CONSEQUENCES
}

By

Kimberley Eve Ballantyne

\author{
A thesis \\ submitted to Victoria University of Wellington \\ in fulfilment of the requirements for the degree of \\ Master of Science \\ in Psychology
}

Victoria University of Wellington

2012 


\begin{abstract}
This thesis describes the development and testing of a theoretically plausible model of antecedents and consequences of workplace interpersonal mistreatment using archival data $(n=10697)$ of civilian and military employees. The sample was split into calibration and validation samples. Principle component and confirmatory factor analysis revealed a complex structure for the workplace interpersonal mistreatment construct across three types of behaviour, and across observed and experienced mistreatment. Furthermore, a total of 17 robust factors were identified in the survey, of which a subset of eight factors was used for developing the model of antecedents and consequences of WIM. The model was tested and refined using regression and structural equation modelling in two samples and validated in a third sample. Individual (seniority), workplace (directive leadership, equity and diversity climate, and health and safety climate) and organisational features (aligned-cohesive culture, service culture) all predict mistreatment. Outcomes of mistreatment include stress, organisational commitment, job satisfaction and leaving intentions. The model showed good fit in the validation sample and is therefore likely to generalise to the population. Implications for organisations and recommendations for future research are discussed.
\end{abstract}




\section{ACKNOWLEDGEMENTS}

I would like to thank Dr Ronald Fischer for supervising this project and providing useful advice and helpful encouragement throughout.

I am grateful to my colleagues at the New Zealand Defence Force, particularly those in the Directorate of Psychology and the Organisational Research and Development unit for supporting me in this research, as well as the Army Education Board for funding my study.

I greatly appreciate the moral support provided by my husband, family and friends while I undertook this thesis.

Lastly, I would like to thank the members of the New Zealand Defence Force who, by taking part in the Ongoing Attitude Survey, allowed this research to take place. 


\section{CONTENTS}

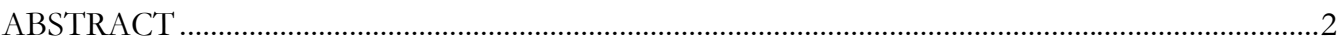

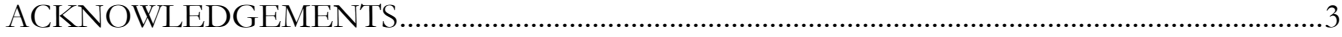

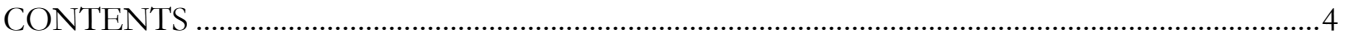

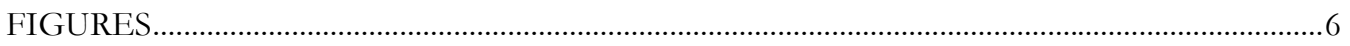

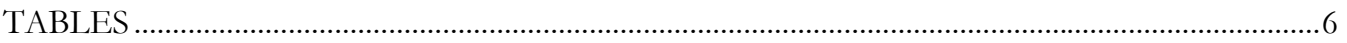

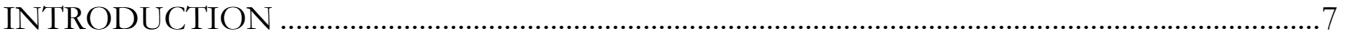

Definitions of Workplace Interpersonal Mistreatment...................................................................

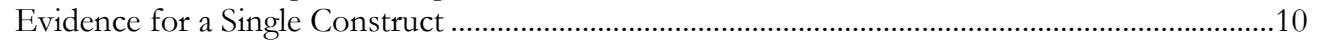

Theoretical Models of WIM..........................................................................................................11

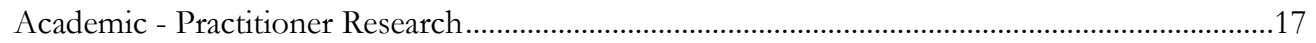

Empirical Evidence for Research Hypotheses.............................................................................18

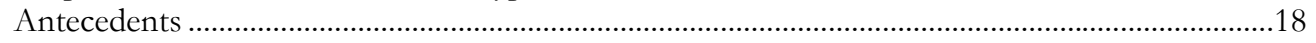

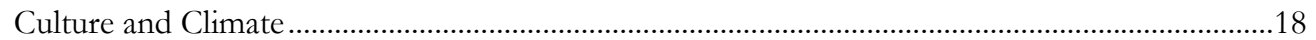

The Importance of an Aligned-Cohesive Culture in the Military............................................................20

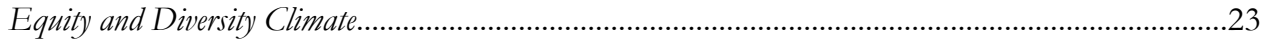

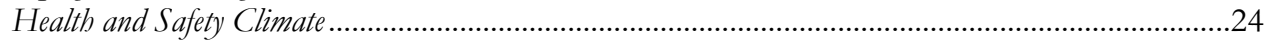

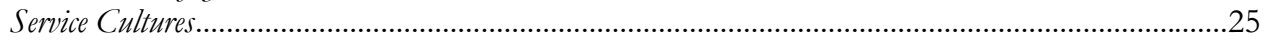

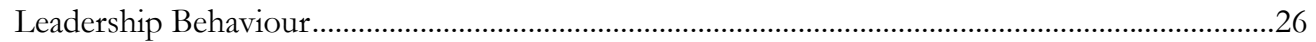

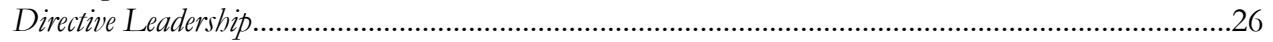

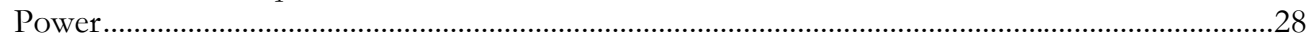

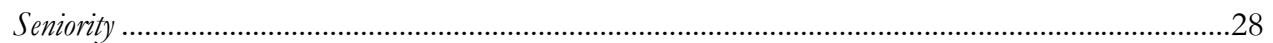

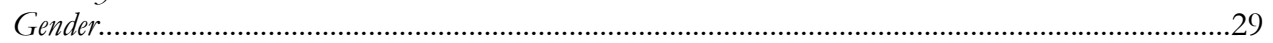

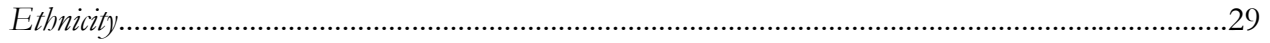

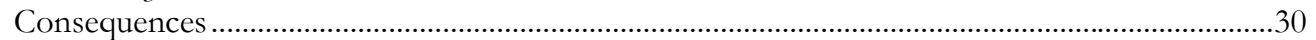

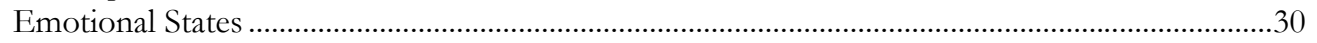

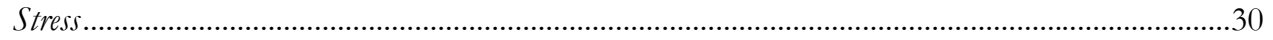

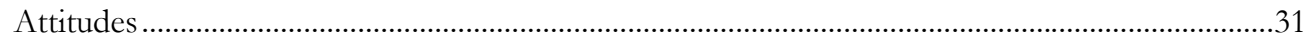

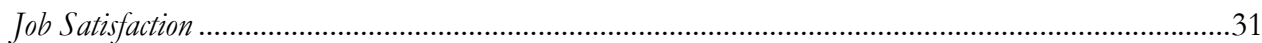

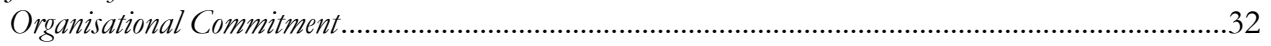

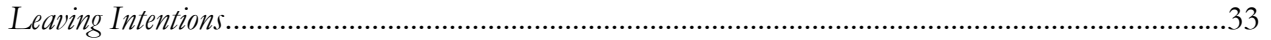

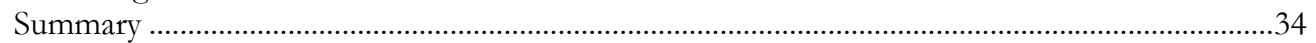

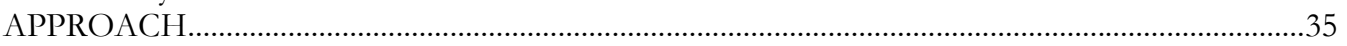

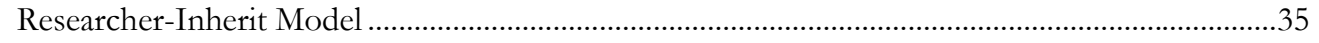

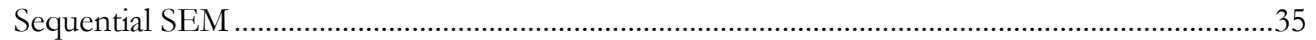

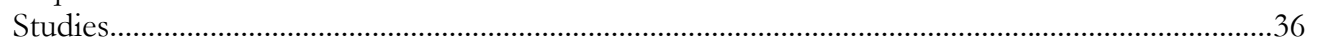

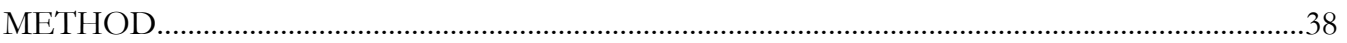

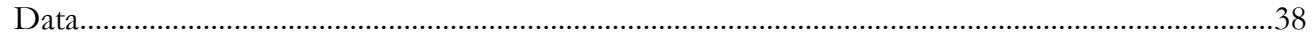

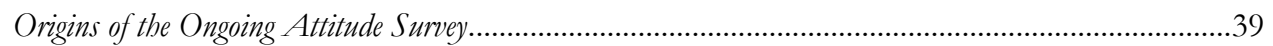

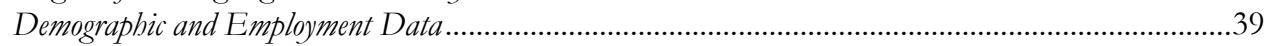

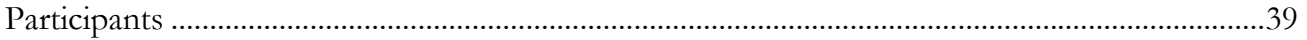

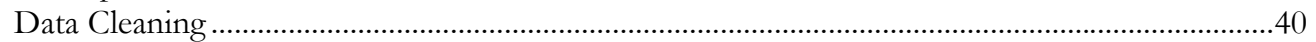

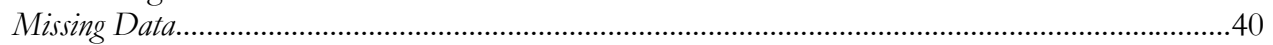

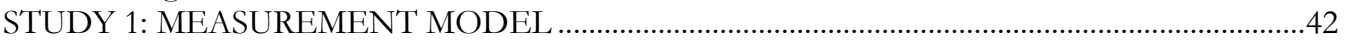

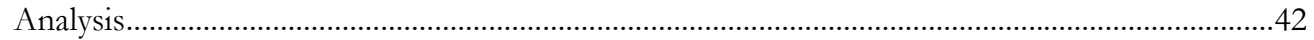

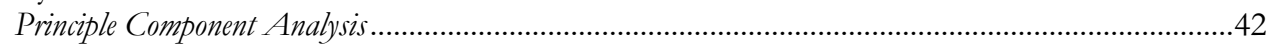

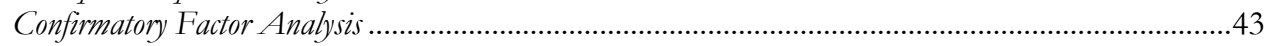

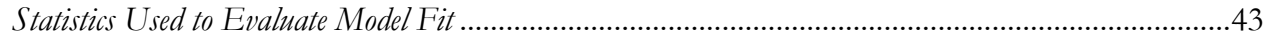

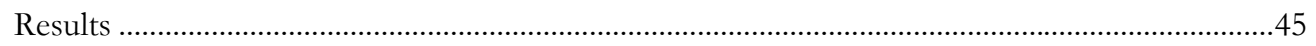

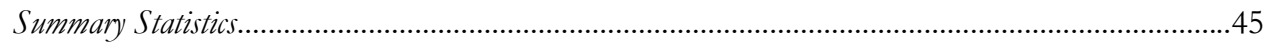

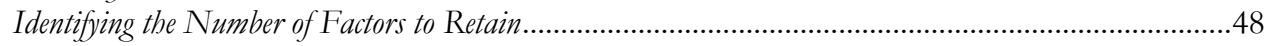

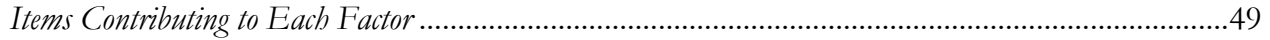

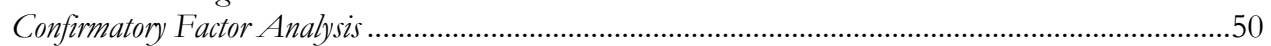

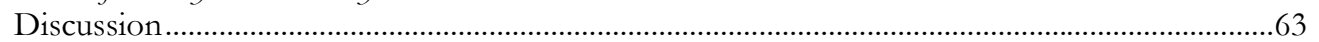

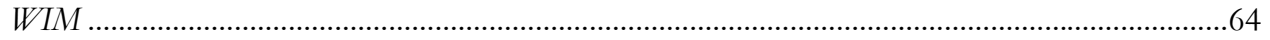

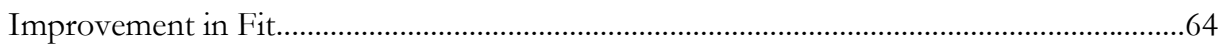

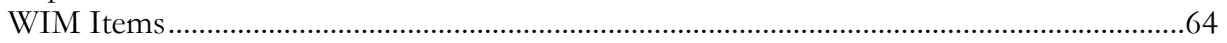

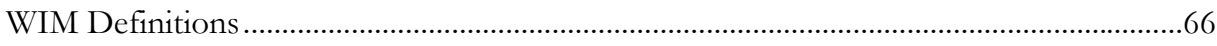


Literature Relevant to the Structure of WIM .........................................................................67

Subjective and Objective Approaches to WIM Measurement...............................................70

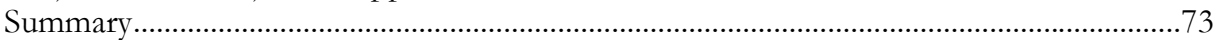

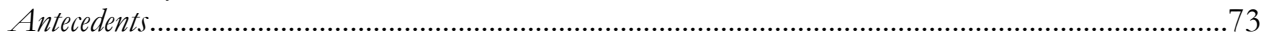

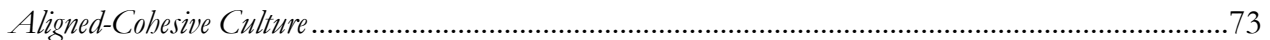

Improvements in Fit.........................................................................................................

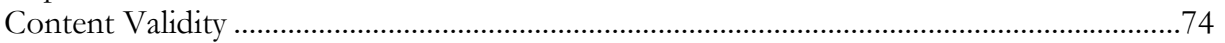

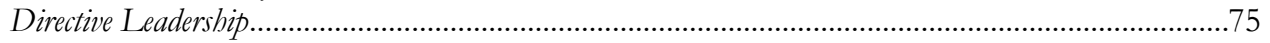

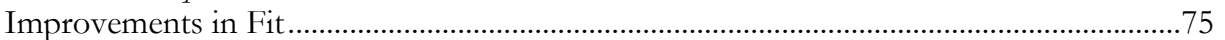

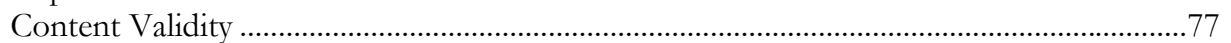

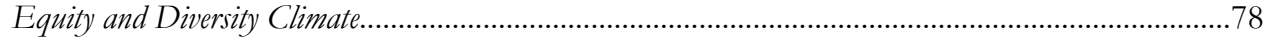

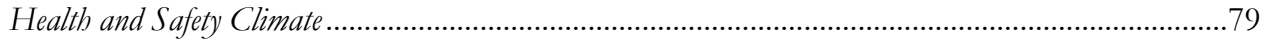

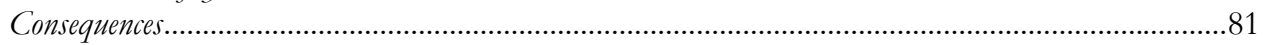

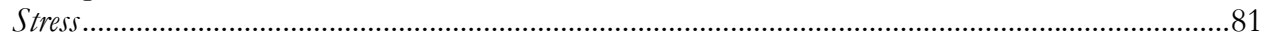

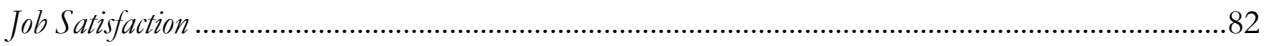

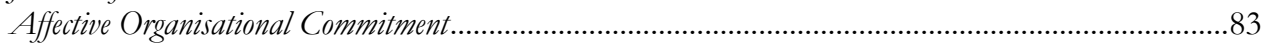

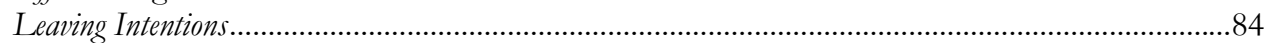

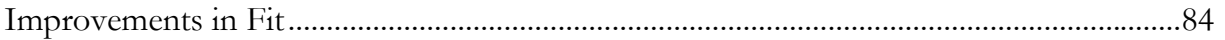

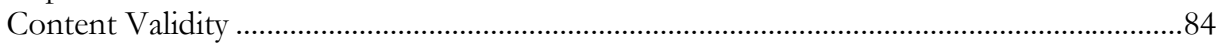

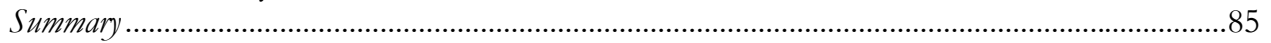

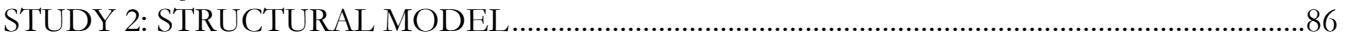

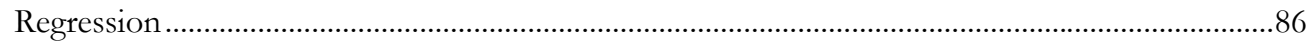

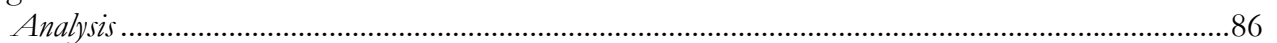

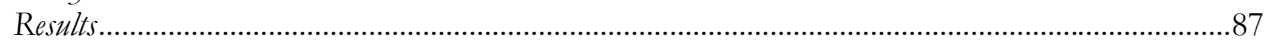

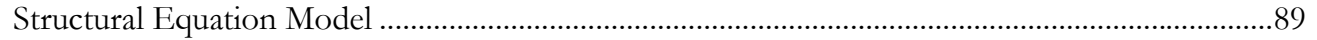

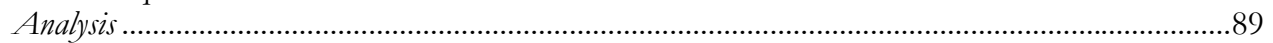

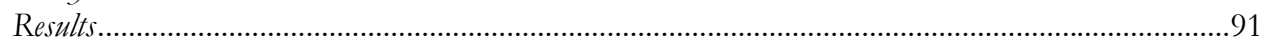

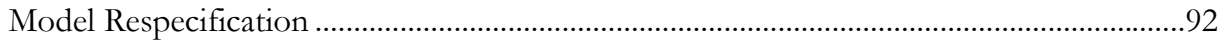

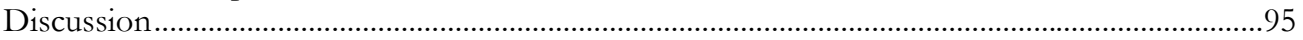

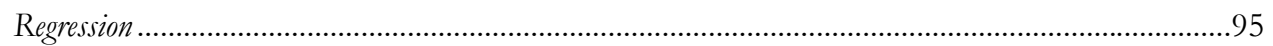

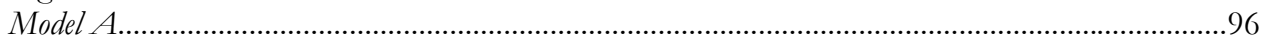

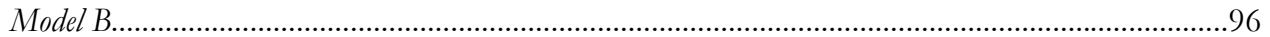

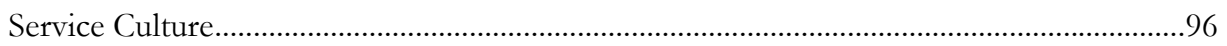

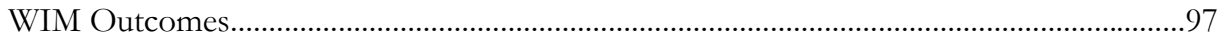

Direct Effects of Antecedents on Outcomes...................................................................99

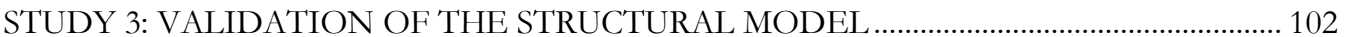

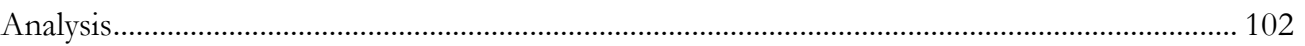

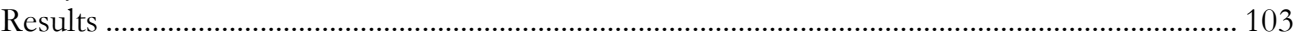

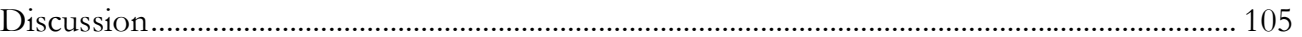

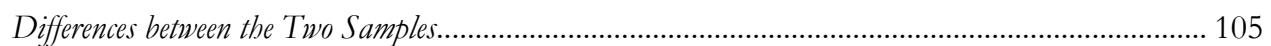

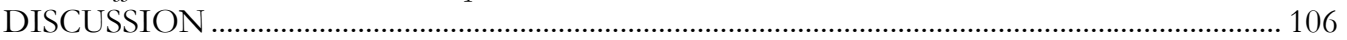

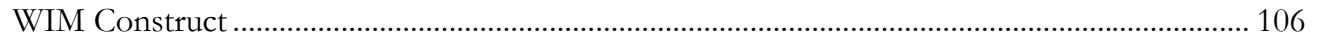

Model of Antecedents and Consequences.............................................................................. 107

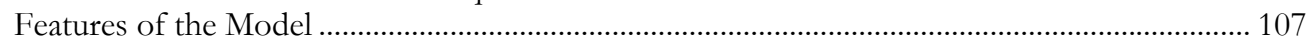

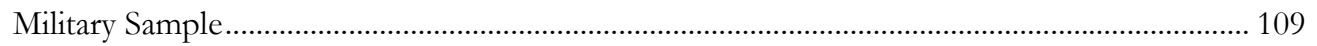

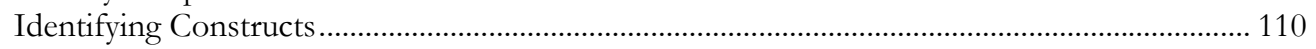

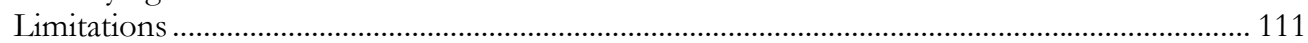

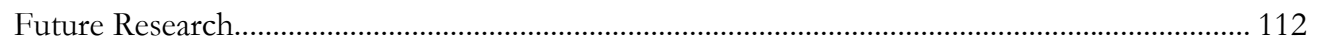

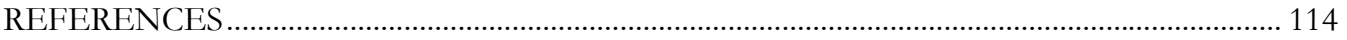

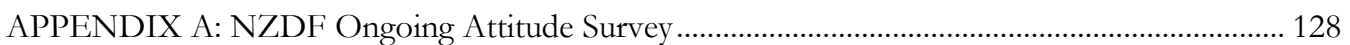




\section{FIGURES}

Figure 1: Einarsen, Hoel, Zapf \& Cooper's (2003) theoretical framework for the study and

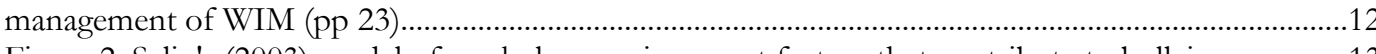

Figure 2: Salin's (2003) model of workplace environment factors that contribute to bullying ..............13 Figure 3: Framework for research on the workplace antecedents and consequences of WIM ..............16

Figure 4: Approach to research using multiple samples .............................................................................3

Figure 5: Parallel analysis to identify number of factors for retention .....................................................48

Figure 6: Close up of parallel analysis to identify number of factors for retention ..................................49

Figure 7: Unexplained variance in the WIM measure.................................................................................65

Figure 8: Hypothesised model A: fully mediated model ...........................................................................90

Figure 9: Standardised B coefficients for Model B sample 2 (2007) ........................................................93

Figure 10: Relationships among constructs in Model B, sample 3, 2009 .............................................. 104

\section{TABLES}

Table 1: Strengths and weaknesses of existing theoretical models of WIM ............................................15

Table 2: Number of participants in each of the three datasets ..................................................................40

Table 3: Descriptive statistics and correlation among the study variables for sample 1 .........................45

Table 4: Descriptive statistics and correlation among the study variables for sample 2 .........................47

Table 5: Item loadings for each factor in the PCA and CFA ................................................................51

Table 6: Construct fit statistics from PCA and CFA .............................................................................60

Table 7: Components of the WIM measure............................................................................................64

Table 8: Aspects of alignment and cohesion as measured by 'aligned-cohesive culture' .........................75

Table 9: Directive and transactional leadership items.............................................................................77

Table 10: Health and safety climate items compared to Psychological Safety construct..........................80

Table 11: Predicting WIM ........................................................................................................................8

Table 12: Standardised B coefficients for antecedents and consequences of WIM, model A ...............92

Table 13: Variance in endogenous constructs explained by Model B (sample 2, 2007 data) ..................94

Table 14: Descriptive statistics and correlation among the study variables for sample 3 ..................... 103

Table 15: Variance in endogenous constructs explained by the model (sample 3, 2009 data)............. 104

Table 16: Proportion of females in samples 2 and 3 .......................................................................... 105 


\section{INTRODUCTION}

Workplace interpersonal mistreatment (WIM) describes a collection of behaviours which have been of concern to organisations for decades (Einarsen, Raknes, \& Matthiesen, 1994). Although legislation exists to protect employees from such discriminatory, harassing and bullying behaviours (in New Zealand, e.g. Human Rights Act, 1993; Harassment Act 1997; Employment Relations Act, 2000; Crimes Act, 1961; Privacy Act, 1993), prevalence estimates continue to be between $9 \%$ and $71 \%$ (Barling, Dupre \& Kelloway, 2009). Much uncertainty exists as to the exact nature of WIM as a construct (Willness, Steel \& Lee, 2007). This thesis contributes to this debate as it offers a psychometric test of the WIM construct across multiple behaviours (discrimination, harassment and bullying) and across observers and targets in a large data set.

Researchers interested in WIM have attempted to identify its antecedents, with the hope of reducing its prevalence (e.g. Notelaers, De Witte \& Einarsen, 2010; Einarsen, 1999). The negative outcomes of WIM for both the organisation and the individual have also been studied (e.g. Yildirim, 2009; Raver \& Nishii, 2010; Ensher, Grant-Vallone \& Donaldson, 2001). However, much of this existing research focuses on either antecedents or consequences of WIM (e.g. Sims, Drasgow \& Fitzgerald, 2005; Bildt, 2005; for an exception see Hutchinson, Wilkes, Jackson \& Vickers, 2010), or only on certain sub-types of WIM (such as harassment e.g. Willness, Steel \& Lee, 2007, Fitzgerald, Drasgow, Hulin, Gelfand, \& Magley, 1997; Fitzgerald, Drasgow, \& Magley, 1999, or gender discrimination e.g. Nye, Brummel \& Drasgow, 2009) and/or is not guided by theory (c.f. Willness, Steel \& Lee, 2007). This thesis makes an important contribution to our understanding of WIM in organisations as it tests a theoretically plausible model of both the antecedents and consequences of WIM together.

Some WIM researchers have focused on military organisations due to the military's unique culture and legislative position (e.g. Fitzgerald, Drasgow, \& Magley, 1999; Mageroy, Lau, Riise, \& Moen, 2009; Munson, Miner, \& Hulin, 2001). However, little research addressing workplace interpersonal mistreatment in the military has been 
conducted in New Zealand (for an exception, see Winsborough, Morris, \& Hughes, 2008). This thesis considers WIM in the New Zealand military context.

Heeding calls by Bartunek (2007); Hinkin, Holtom, and Klag (2007); Anderson, Herriot and Hodgkinson, (2001) to use a collaborative research approach this thesis uses existing military data from an organisational attitude survey. As such, a practical contribution of this thesis is that it identifies the constructs measured in the organisational survey so as to determine psychometrically valid measures of important organisational constructs including WIM and its antecedents and consequences. The factors identified and described in this thesis can be used for further studies using this data base.

\section{Definitions of Workplace Interpersonal Mistreatment}

Workplace interpersonal mistreatment (WIM) is an umbrella term which connotes milder behaviours such as workplace incivility and more severe behaviours such as workplace aggression. I will discuss three common definitions of WIM which address intent, the cessation of positive behaviours and the range of severity of WIM behaviours. The three definitions are: (1) incivility; (2) workplace aggression; and (3) interpersonal mistreatment.

Workplace incivility has been defined by Andersson and Pearson (1999) as:

"low-intensity deviant behavior with ambiguous intent to harm the target, in violation of workplace norms for mutual respect. Uncivil behaviors are characteristically rude and discourteous, displaying a lack of regard for others" (p. 457).

Incivility behaviours are low-level but common behaviours which can have important effects for organisations and individuals. In contrast workplace aggression can include violence which encompasses low-incidence but dangerous behaviours such as sexual harassment, and the extremes of rape and murder.

Workplace aggression defined by Neuman and Baron (1998) as: 
"efforts by individuals to harm others with whom they work, or have worked, or the organizations in which they are presently, or were previously, employed' ( $\mathrm{p}$. 395).

The distinction between these two types of WIM is the intent to harm. The intent to harm is clearly established in workplace aggression, but ambiguous in cases of incivility.

The term workplace interpersonal mistreatment was coined by Cortina and Magley (2003) who define interpersonal mistreatment as:

'a specific, antisocial variety of organizational deviance, involving a situation in which at least one organizational member takes counternormative negative actions — or terminates normative positive actions — against another member.... Interpersonal mistreatment can thus range from subtle social slights to general incivility to blatant harassment and violence.' (p. 247)

The Cortina and Magley definition is distinctive because it includes the cessation of positive behaviours as part of the definition. This extends the scope of behaviours beyond those included in other definitions to activities such as discrimination during a promotion process, which are not explicitly included in other terms which are more narrowly defined.

Some of the literature, particularly the aggression literature (e.g., Bjorkqvist, Osterman, \& Hjelt-Back, 1994) uses definitions that include an intention requirement: that is the behaviour in question must have been intentionally harmful. The intention requirement is unhelpful because it is often impossible to retrospectively assess the perpetrators intention. Workplace interpersonal mistreatment is therefore a useful concept as the intent to harm is not a key inclusion criterion. Rather, the focus is the harm that has occurred. This broad definition includes unintended behaviour as well as clearly intentional and serious behaviour. When using self-reported measures of perceived WIM, the absence of an "intention" requirement is important.

These three definitions give a flavour for the wide ranging terminology used in the literature in this area. This diversity of terminology is primarily due to the research 
being driven by socially observed phenomena rather than by theory. In addition, research is conducted in a number of distinct disciplines (e.g. psychology, management, education).

The range of terminology used is also demonstrated by meta-analyses, for example, Lapierre, Spector, and Leck (2005) used 18 search terms, including bullying, harassment, incivility and aggression to identify relevant literature in a study of sexual and non-sexual workplace aggression. A meaningful discussion of WIM behaviour in organisations requires these broad ideas to be conceptualised using a single term. Conceptually, the label workplace interpersonal mistreatment serves this function best.

\section{Evidence for a Single Construct}

Although historically research into various types of WIM has occurred under many different names, recent researchers have called for work to identify the degree of overlap among the types of WIM. Willness, Steel and Lee (2007) specifically call for research which investigates the construct development of WIM, and a focus on the similar aetiologies and affects of the different sub-types of WIM.

Recent conceptual work focusing on the similarities across the different types of WIM has had mixed results. Griffin (2009) found evidence for a single construct underlying incivility, emotional abuse, interpersonal aggression, bullying, and social undermining.

Lim and Cortina (2005) investigated the overlap between workplace gender harassment, sexual harassment and general incivility and found that these three areas were highly correlated. The authors concluded that the more extreme forms of sexual harassment occur in a workplace characterised by general disrespect.

Raver and Nishii (2010), Berdahl and Moore (2006) and Bildt (2005) have all investigated the co-occurrence of types of WIM from a discrimination perspective. Some evidence for the co-occurrence of these behaviours has been found. Raver and Nishii (2010) found that three types of workplace harassment (gender, ethnic and general workplace harassment) were moderately correlated. Bildt (2005) found that there were links between gender discrimination, bullying, conflicts and sexual 
harassment, and these links were stronger among women than men. At an individual level Berdahl and Moore (2006) found that ethnic and gender harassment co-occur.

In the bullying literature, research on police civilian workers and a general sample of civil servants found a four factor oblique model for four components of bullying: task attack, personal attack, isolation and verbal attack. These results indicated that the latent variables are distinct constructs but are moderately to strongly correlated with one another (Dick \& Rayner, 2004).

The inconclusive nature of the findings on the co-occurrence of different types of WIM suggest that there may be one important construct underlying these multiple areas, or these behaviours may be related to one another in more complex ways. This thesis contributes to this literature as it investigates the structure of WIM using exploratory and confirmatory factor analytic techniques in a large military sample.

\section{Theoretical Models of WIM}

Researchers have noted that the WIM literature is not strongly theory driven and have hypothesised that this may be due to the topic being a social phenomenon and of practical rather than theoretical interest (Willness, Steel \& Lee, 2007). Academic research in this area was originally initiated as a result of litigation relating to WIM and the need to address this important social problem rather than an academic interest in conceptualising WIM. However, some theories have been developed and these focus either on the process of WIM or on the wider organisational and social contexts that influence, and are influenced by, WIM. Theoretical models that focus on the process of WIM have identified the role of organisational justice judgements (Bowling \& Beehr, 2006), attribution processes (Hershcovis et al., 2007) and appraisal processes (Bergman, Langhout, Palmieri, Cortina \& Fitzgerald, 2009). These theories are concerned with the interpersonal and individual cognitive processes of victims. As such, they are less concerned with managerial practices.

A wider sociocultural approach which considers the antecedents and consequences of WIM proves useful for both practitioners in organisations and researchers (Einarsen, Hoel, Zapf \& Cooper, 2003). A sociocultural approach focuses on understanding the situational factors that influence behaviour and the implications 
for organisations and society rather than just the individual level or interpersonal phenomena. Adopting this sociocultural model, some authors have asserted that as WIM is embedded within the culture of the organisation, the primary responsibility for change lies with the organisation (Monks, Smith, Naylor, Barter, Ireland \& Coyne, 2009). They argue that the organisation is therefore uniquely placed and empowered to influence WIM at levels which individuals simply cannot. Factors which are under an organisation's control may therefore be a better focus for research efforts which aim to bring about practical change. Three influential sociocultural models of WIM, and their strengths and weaknesses will be discussed.

Einarsen, Hoel, Zapf and Cooper (2003) propose a theoretical framework for both studying and managing WIM that takes into account sociocultural factors, organisational and individual factors:

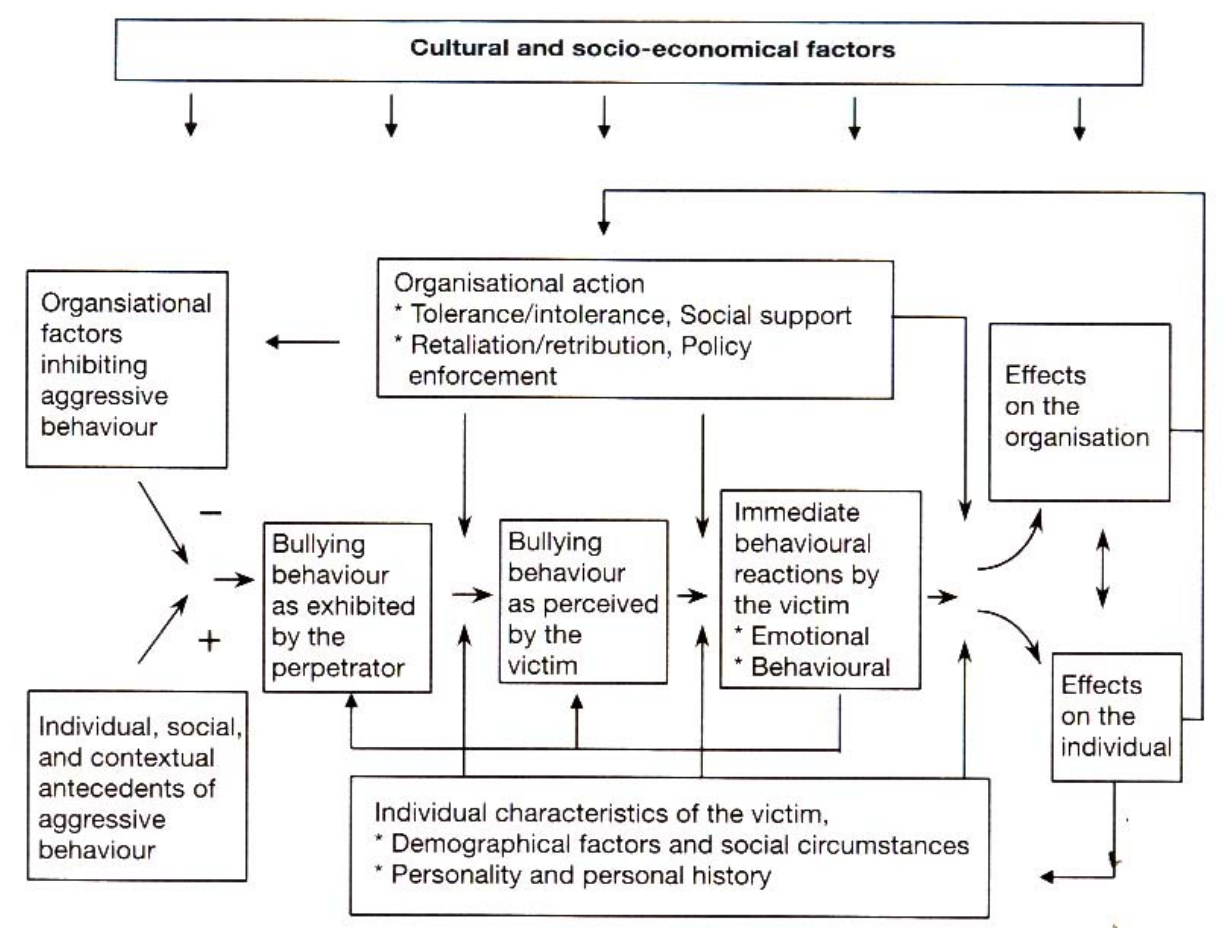

Figure 1: Einarsen, Hoel, Zapf \& Cooper's (2003) theoretical framework for the study and management of WIM (p.23).

A strength of the Einarsen, Hoel, Zapf and Cooper (2003) model is that it conceptualises the recursive nature of many of the factors. For example, the effects of WIM on the organisation (such as increased turnover or costs associated with litigation) may in turn influence the action taken by the organisation, and the 
organisational culture. However, this model is very comprehensive and complex, therefore making it difficult to test empirically in its entirety.

Salin's (2003) conceptual model focuses on the antecedent work environmental factors. The model makes the distinction between necessary but not sufficient antecedents by grouping antecedents into three groups: enabling, motivating, and precipitating factors. Enabling factors may allow WIM to occur but are not sufficient to bring WIM about alone. Examples of enabling factors include perceived power imbalance, and dissatisfaction with work control. Motivating factors are factors which make WIM a worthwhile behaviour for personnel to undertake and include factors such as high internal competition and reward systems. Precipitating factors could be related to changes in the organisation which create a stressful or insecure environment which may make WIM more likely to actually evolve.

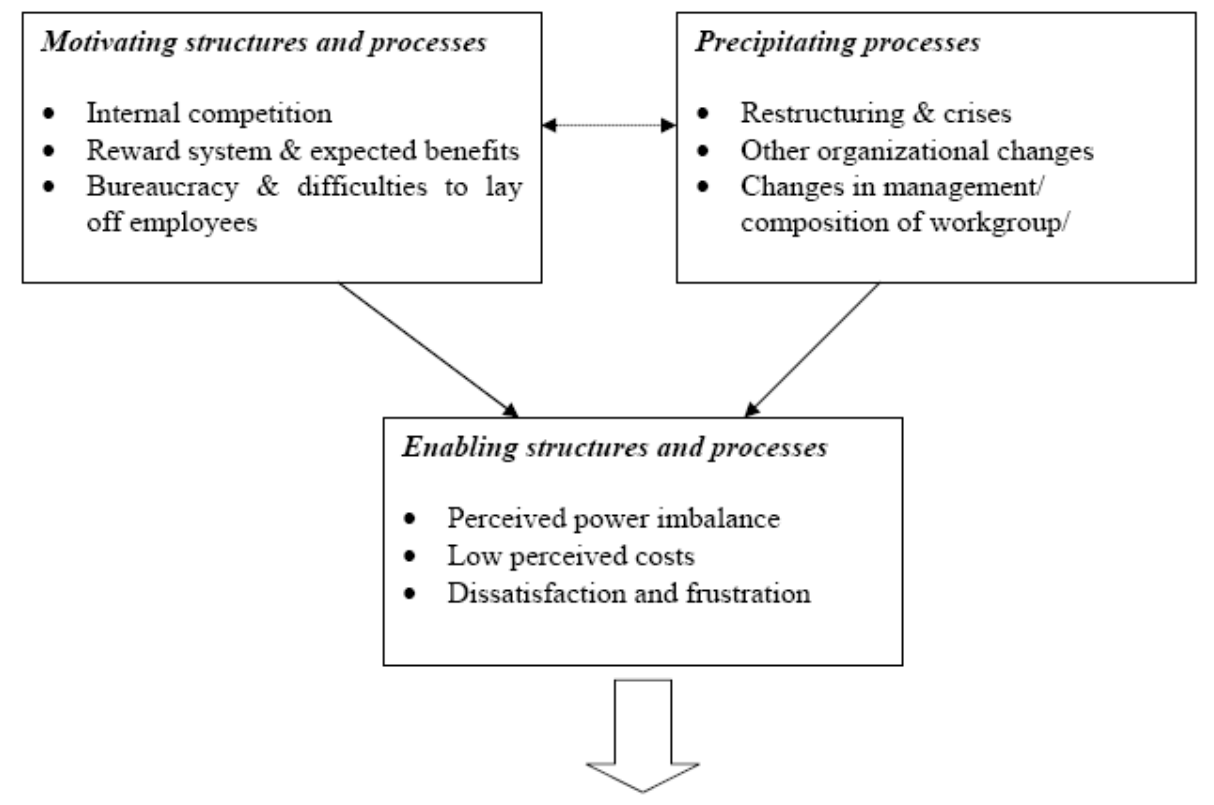

\section{BULLYING POSSIBLE AND MORE}

LIKELY

Figure 2: Salin's (2003) model of workplace environment factors that contribute to bullying

A weakness of the Salin (2003) model is that the antecedent factors are not conceptualised from an organisational culture or climate perspective. Many of the enabling and motivating factors are part of an organisation's culture. Organisational culture provides a functional way of conceptualising the complex influence of work environment factors on WIM behaviours. By not considering antecedent factors from an organisational culture or climate perspective, Salin's (2003) model neglects 
an important and meaningful conceptualisation of the workplace environment which is theoretically (Schein, 2005) and practically (Gillespie, Denison, Haaland, Smerek \& Neale, 2008) useful.

The Leymann model is another conceptual model of the antecedents of bullying that has heavily influenced the European tradition of WIM research. The model includes four features of the work environment that elicit WIM behaviours (Leymann, 1996; Einaresen, Hoel, Zapf \& Cooper, 2003):

1. the socially exposed position of the victim,

2. deficiencies in leadership behaviour,

3. poor work-design and,

4. low departmental morale.

The feature 'low departmental morale' is operationalised in a similar way to the domains of culture and climate (Einaresen, Hoel, Zapf \& Cooper, 2003). A strength of the Leymann model is that includes features of the work environment at multiple levels in the organisation: individual (socially exposed position of the victim), team (deficiencies in leadership behaviour) and organisation (low departmental morale) levels.

Salin's (2003) model and the Leymann model (Lemann, 1996; Einaresen, Hoel, Zapf \& Cooper, 2003) provide useful ways of conceptualising of WIM as they focus on the organisation but they do not provide an account of the outcomes of WIM. Understanding the outcomes of WIM is important from the perspective of organisations, as this is a key means by which organisations are motivated to make changes.

Table 1 summarises the strengths and weaknesses of existing theoretical approaches to the antecedents and consequences of WIM. 
Table 1: Strengths and weaknesses of existing theoretical models of WIM

\begin{tabular}{|c|c|c|}
\hline & Strengths & Weaknesses \\
\hline \multirow[t]{2}{*}{ Einarsen } & Includes outcomes & Complex \\
\hline & & Difficult to test in its entirety \\
\hline \multirow[t]{2}{*}{ Salin } & $\begin{array}{l}\text { Focuses on antecedents under } \\
\text { an organisation's control }\end{array}$ & Does not include outcomes \\
\hline & & $\begin{array}{c}\text { Features are not conceptualised } \\
\text { in relation to culture. }\end{array}$ \\
\hline \multirow[t]{2}{*}{ Leyman } & $\begin{array}{l}\text { Focuses on antecedents under } \\
\text { an organisations control }\end{array}$ & Does not include outcomes \\
\hline & $\begin{array}{l}\text { Includes antecedents at } \\
\text { individual, team and } \\
\text { organisational levels } \\
\end{array}$ & \\
\hline $\begin{array}{l}\text { Interpersonal Models (e.g. } \\
\text { Bowling \& Beehr, 2006, } \\
\text { Hershcovis et al., 2007) }\end{array}$ & $\begin{array}{c}\text { Include mechanisms by which } \\
\text { outcomes occur }\end{array}$ & $\begin{array}{l}\text { Focuses on the individual or } \\
\text { interpersonal level, excluding } \\
\text { social context and culture }\end{array}$ \\
\hline
\end{tabular}

As indicated in table 1, all existing models contain some weaknesses. In order to progress our understanding of the nature and consequences of WIM a new theoretical model is required that has the following features: (1) it focuses on antecedents which are primarily under the organisation's control, therefore offering best opportunities for change; (2) it includes outcomes for both organisations and individuals; (3) it focuses on all three levels of an organisation: individuals, teams and organisation; (4) it is testable with data from a large complex organisation; and (5) it includes the hypothesised mechanisms of how antecedents and consequences are related to WIM.

This new model is proposed in Figure 3 and further explained in sections on the antecedents and consequences of WIM below. 


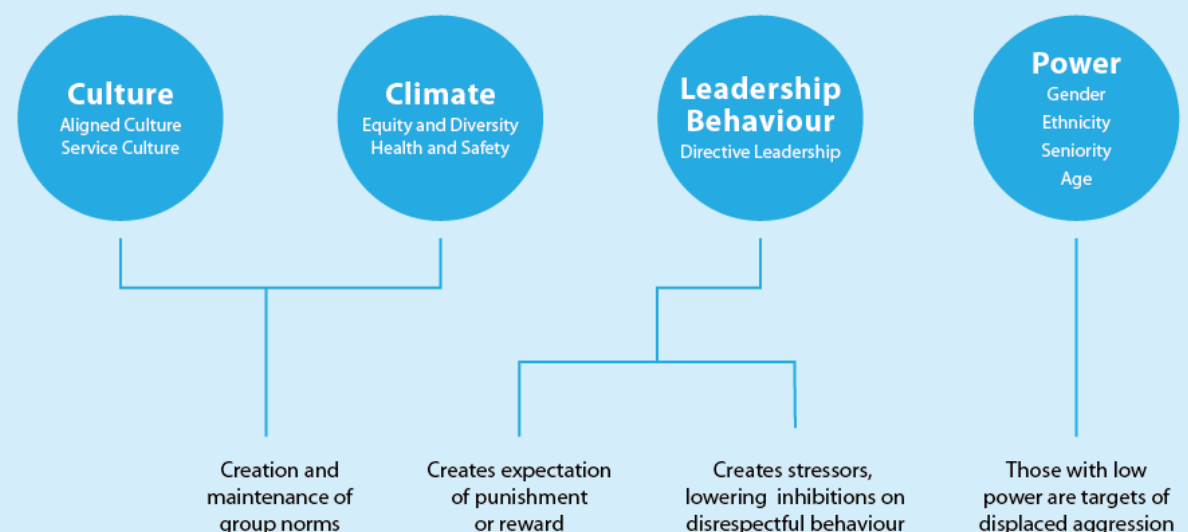
group norms
disrespectful behaviour
displace targets of

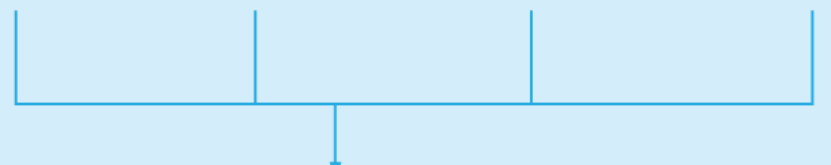

WIM

Observed Misreatment

Experienced Mistreatment

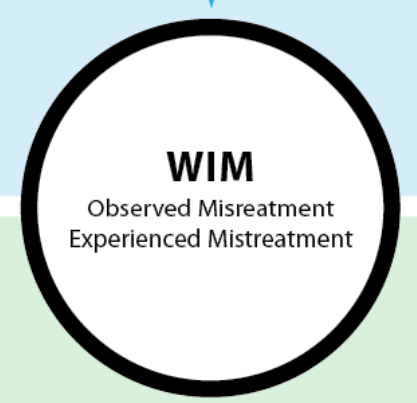

Social ostracism, violation of expectations

and schemas

workplace due to
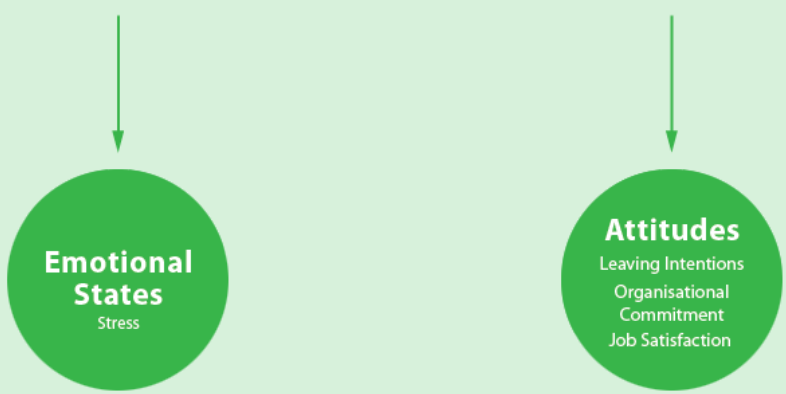

Figure 3: Framework for research on the workplace antecedents and consequences of WIM 


\section{Academic - Practitioner Research}

The theoretical model posed in figure 3 has been tested in the current study by using an existing survey developed by practitioners. The research approach used in this thesis is therefore an example of collaborative academic-practitioner research. Academic-practitioner research has been called for by many authors (e.g. Bartunek, 2007; Hinkin, Holtom, \& Klag, 2007; Anderson, Herriot \& Hodgkinson, 2001) as it meets both the relevance and rigour requirements of quality organisational research. Anderson, Herriot and Hodgkinson's (2001) describe four models of organisational research- (1) pragmatic research (high on rigour and relevance) the gold standard of organisational research; (2) pedantic research - high rigour, low relevance (a tendency of academics) ; (3) popularist research - low rigour and high relevance (a tendency of practitioners; and (4) puerile research (low rigour and low relevance). Collaborative academic-practitioner research is an example of pragmatic research in the Anderson, Herriot and Hodgkinson matrix.

One method of pragmatic academic practitioner research is the researcher-inherit model as defined by Robert Vandenberg in Hurley, et al., (1997):

\section{"the researcher inherit [s the] organisation's database representing an in- house developed survey in which items are anchored to known conceptual bases." (p 675).}

The benefits of this type of research have been noted by McCall and Appelbaum (1991) and include that researchers gain access to interesting participant groups, large sample sizes, and often datasets that have numerous potential correlates. These benefits are balanced against the limitations of such research. For example, the dataset necessarily constrains and drives the development of the scientific questions. Existing datasets tend to cover many domains superficially rather than fewer domains in more depth. The whole process of researcher-inherit research is recursive and research questions often have to be reformulated. Therefore, researcher-inherit studies are primarily statistical in nature as there is no data collection. McCall and Appelbaum (1991) note that the outcomes of such studies are limited by the 'statistical creativity, expertise and energy that is devoted to analyzing the data' (p. 
916). This approach differs from traditional academic research, which within a single study, is linear and unidirectional.

The research presented in this thesis is an example of collaborative academicpractitioner research. As such, the research questions are constrained by the data available in the pre-existing survey. The hypotheses developed below are driven by the available data and not driven by a comprehensive theory. The research questions have been reformulated a number of times to accurately fit the data available. Variables available in the dataset that can be expected to have some conceptual relationship with WIM are discussed. These constructs are relatively broad and may not capture some finer distinctions made in the literature. There are also other relevant variables identified in the literature (e.g. role stressors (Einarsen, Raknes, \& Matthiesen, 1994) and environmental clarity (Bowling \& Beehr, 2006)) for which no data was available.

\section{Empirical Evidence for Research Hypotheses}

Evidence in support of the theoretical model pictured in figure 3 exists in two forms: explanation of the theoretical mechanisms linking WIM with its antecedents and consequences, and empirical support demonstrating the existence of these relationships. These two forms of evidence are described for each of the components of the model: culture and climate, leadership behaviour, power, emotional states, and attitudes. As culture and climate share a single mechanism of influence these two components are discussed together.

\section{Antecedents}

\section{Culture and Climate}

Both the culture of an organisation and the climate of specific workplaces are strong determinants of how personnel within the organisation behave. New personnel are inducted into the culture of the workplace and explicitly and implicitly advised about 'how things are done around here' (Schein, 2004). Personnel who are not able to integrate into the culture may stay and experience difficulty, or leave. This influence of organisational culture and climate on individual behaviour operates through the creation and maintenance of group norms. Group norms are shared understandings 
of what is and is not acceptable within a group of people in a certain setting. The group's understanding of the content of norms can differ from the stated behavioural standards determined by organisational policies (Keashly \& Jagatic, 2003).

The influence of organisational culture and climate on WIM has been proposed to occur by two mechanisms (Brodsky, 1976). The first is that WIM behaviours are used in the organisation as a means of increasing productivity and performance. This use is based on the belief that personnel perform best when motivated by fear. Therefore, the threat of WIM behaviours by management motivates personnel to perform in order to stay out of the 'firing line' (Keashly \& Jagatic, 2003). This explanation is likely to be reflected in the content of both policy and behaviour. Alternatively, individuals within the organisation may undertake WIM to serve their own aims with the belief that they have implicit permission from management to do so. The implicit condoning of WIM behaviour is communicated by the failure of organisational leaders to react to instances of WIM when they occur, even if formal organisational policy dictates that such behaviour is unacceptable (Hoel \& Salin, 2003; Lewis \& Rayner 2003).

Some research into how the content of different organisational cultures affects WIM has been conducted. Keashly and Jagatic (2000) focused on the interpersonal components of organisational culture - involvement, morale, teamwork and supervision, across a representative sample of American workers from a range of organisations. All four components were negatively related to reported WIM indicating that the quality of interpersonal relations in an organisation is a feature of culture that predicts WIM behaviour. Keashly and Jagatic's research focuses on the common features of cultures which impact of WIM across organisations. An alternate way of investigating the influence of organisational culture on WIM is to consider the specific cultural content that an organisation requires to operate in a given context.

The content of an organisation's culture and climate is influenced by the type of work the organisation performs. In other words, the environment the organisation operates in and the type of work the organisation's members undertake both influence the organisational culture. 


\section{The Importance of an Aligned-Cohesive Culture in the Military}

WIM research involving military samples has tended to focus on the negative features of military culture that impact WIM. For example, job-gender context research indicates that workplaces where one gender is a minority, or where the occupation is traditionally held by one gender have more WIM (Fitzgerald, Hulin \& Drasgow, 1995). The military is one of the more extreme examples of an occupation that is traditionally dominated by one gender. A second factor of military culture that has been studied is the entrenched and acute hierarchy within the military. Ilies, Hauserman, Schwochau and Stibal (2003) found that the military demonstrated the most extreme example of high power differentials, of all workplaces included in their study, and therefore concluded that the military is at unique risk of WIM behaviour. Archer (1999) identified features of paramilitary culture which may contribute to WIM such as the hierarchical rank structure, the authoritarian leadership and the emphasis of conformity. Contrastingly, the way that military culture may influence the reduction of WIM has not been well studied.

Both the negative and positive features of military culture result from the specific type of work that the military is engaged in. Military work has three aims: 1) the distribution and application of military means to achieve governmental objectives; 2) the coordination of the logistics of battle; and 3) the preparation of soldiers to perform successfully in combat (Liddell Hart, 1967; Taylor, 2003). Military operations require complex strategy and tactics to coordinate numerous individuals and a range of equipment to achieve governmental aims. The logistics of war fighting are complicated, primarily due to the requirement to be self-sustaining. Militaries cannot rely on external sources to provide necessities such as food, clothing and ammunition. Instead, all of these supplies must be self-sustained. A culture that facilitates coordination and integration between different parts of the organisation is critical to achieving these first two aims. The third facet of military work is preparing soldiers for combat. Combat has been described as 'the province of hardship and suffering, of stress and fear and pain and death.' (van Creveld, 1989, pp 313-4). Success in combat is principally about the soldier's ability to cope with these psychological states (van Creveld, 1989). Military training is therefore intentionally demanding and service personnel are subjected to psychological stressors that employees in other organisations are not. A separation necessarily exists between the 
standards of behaviour acceptable in a military environment and those acceptable in a civilian environment.

The separation of behavioural standards does not suggest that abuse is condoned in the military or that there are no boundaries. On the contrary, the US military has identified a positive equal opportunities climate as being essential for military readiness (Truhon, 2008). To effectively maintain the distinction, it is important that any tough behaviour is justifiable in relation to preparing a soldier for battle. The uniqueness of the military environment produces a situation where its members are distinctly vulnerable; the line between necessary toughness in training and unacceptable abuse is a fine one that is easily crossed (Wither, 2004). Given these circumstances, military leaders must be responsible for ensuring that suitable measures are put in place to minimize opportunities for abuse and make sure that transgressions are dealt with appropriately.

This responsibility falls particularly on military leaders as militaries are protected by, and subjected to, different legislation than other employers. Military service people (in the UK and the commonwealth) serve at 'Her Majesty's Pleasure' and are therefore not protected by standard employment law. This makes the trust relationship between leaders and personnel even more important. For a military to operate successfully, its culture must include social norms which oblige leaders to live up to the responsibility of maintaining the difficult balance between necessary toughness and protecting individuals from unjustified harm. Personnel must believe that leaders will maintain this balance. If personnel do not trust their leaders in this way, they will not consent to making themselves vulnerable to the possible abuse that could occur. Therefore, the shared understanding of the acceptable standards of behaviour is critical to the success of the military as an organisation.

Additionally, modern militaries are comprised of service personnel and civilian employees. The latter is a group that is not well studied. They form an interesting sub-group in military organisations as they are employed within the military environment but are subject to different law (including legislation such as the Employment Relations Act 2002) to military service people. This results in an interesting dynamic, particularly in areas such as WIM. Relevant features of this 
dynamic include: the constant rotation of service personnel through jobs; established promotion pathways for service personnel in contrast to an absence of promotion policy for civilian employees; scenarios where civilians report to service people (most common) or where service people are direct reports of civilians (uncommon) and the different assumptions regarding appropriate management behaviours; as well as the more general team dynamics resulting from a mix of civilian and service experiences. This mix of personnel and expectations requires an organisational culture which can bring these groups with different perspectives together to work successfully towards organisational goals.

The special organisational features of militaries discussed above create the need for a specific organisation culture. Two key components of successful military culture are alignment and cohesion. Alignment in an organisation refers to the way that different subunits of the organisation are coordinated and integrated in order to achieve the organisation's goals (Beehr, Glazer, Fischer, Linton \& Hansen, 2009). Alignment has also been described as the way that 'an organization's design, strategy, and culture are cooperating to achieve the same desired goals' (Semler, 1997; p. 23). These definitions signify two important aspects of alignment; first, that it relates to multiple parts of the organisation and second, that it relates to organisational performance. Cohesion refers to the glue that binds organisational members together. Cohesion results from positive supportive interactions between group members, integration of individuals into the group, and perceptions of fairness in allocation of group resources (Turok, Kearns, Fitch, Flint, McKenzie \& Abbotts, 2006). A cohesive culture emphasizes the importance of support among group members, which balances the operational requirement of tough training. Cohesion has been demonstrated to be related to WIM by New Zealand researchers who found observers and targets of bullying reported worse cohesive work climate (CooperThomas, Catley, Bentley, Gardner, O’Driscoll \& Trenberth, 2011). Additionally, some evidence for the importance of cohesion in military organisation exists in the WIM literature. Cohesion has been found to mediate the relationship between equity and diversity climate on work outcomes in a military population (Walsh, Matthews, Tuller, Parks, and McDonald, 2010). 
A culture which embodies the features of alignment and cohesion will foster group norms which minimise WIM behaviours, as the integrative and supportive facets of alignment and cohesion create an environment where WIM behaviours are not tolerated or rewarded. Personnel who report their experience of the organisational culture as aligned and cohesive will report less WIM.

Hypothesis 1: Aligned-cohesive culture will be negatively related to WIM.

\section{Equity and Diversity Climate}

Equity and diversity climate concerns the implementation and enforcement of stated organisational policies relating to WIM. Equity and diversity climate therefore, refers to environmental factors that can be measured at the workplace level (Walsh, Matthews, Tuller, Parks, \& McDonald, 2010). The domains covered by equity and diversity climate include lack of sanctions against offenders, concerns that complaints will not be taken seriously and perceived risk of complaining (Willness, Steel \& Lee, 2007). The responsibility for creation of a positive equity and diversity climate lies with management. The implementation and enforcement of policies communicate the social norms about acceptable behaviour throughout the organisation (Pryor \& Fitzgerald, 2003). These social norms determine what behaviour is acceptable and what is not. The actual climate can differ from the stated policy regarding WIM (e.g. Williams, Fitzgerald \& Drasgow, 1999) and when this difference occurs and management fails to enforce the stated organisational policy the 'real' social norms that are communicated prevail to influence behaviour. Therefore, equity and diversity climate is important as it is the link between stated organisational policy regarding WIM and WIM behaviours. A negative equity and diversity climate therefore suggests that WIM is likely to occur (Walsh, Matthews, Tuller, Parks, \& McDonald, 2010).

This relationship has been demonstrated empirically. A climate that permits or endorses sexual harassment has been found to be a key predictor of reported sexual harassment incidents. Indeed the presence of a sexual harassment climate was found to be the best predictor of incidents of sexual harassment in the workplace (Willness, Steel \& Lee, 2007). In summary, equity and diversity climate has been linked to WIM 
itself or the outcomes of WIM in both the sexual harassment and discrimination domains, and in civilian and military populations.

Hypothesis 2: Equity and diversity climate will be negatively related to WIM.

\section{Health and Safety Climate}

Health and safety climate is a subcomponent of safety climate which is the 'perceptions of policies, procedures, and practices relating to safety climate in the workplace' (Neal \& Griffin, 2006, p 946).

A climate for health and safety indicates a belief or value held by the organisation that workers' health, safety and wellbeing are important considerations in the workplace. This value is held in addition to the organisation's primary value of productivity. These two values can be in opposition to one another, often when a short term focus is taken; short-cutting safety measures may increase productivity in the short term as time is saved but the long term consequences are likely to be dire, including for productivity. These values can also be in concordance; healthy, happy workers have the capacity to also be more productive as they can focus on their work and are not being distracted by safety concerns or absent from work due to sick leave.

The health and safety climate is a mechanism by which the organisation communicates its values to personnel. These shared values in turn influence the behavioural norms developed in the workplace. If the organisation values the health and safety of workers and the climate reflects this shared understanding WIM behaviours are not tolerated. The expectation that WIM behaviours will not be tolerated can be enough to prevent them from occurring.

Little research exists in this area but recent Australian research has suggested that a sub-factor of health and safety climate, specifically psychosocial safety climate (PSC) is a lead indicator of bullying and harassment in the workplace (Law, Dollard, Tuckey \& Dormann, 2011). PSC is a perceptual measure of the degree to which organisation 
policies, practices and procedures protect worker psychological health and safety. At the individual level PSC was found to be negatively related to incidents of WIM.

The lack of a research history in this area reflects the recent trend in the broadening of the understanding of health and safety to include risks such as WIM. This trend is also reflected in the focus of health and safety legislation. WIM has only recently been recognised as a workplace health and safety hazard. This change to the legislation suggests that WIM may begin to be influenced by health and safety climate. Previously people may have not considered health and safety and WIM to be related, but now WIM behaviours are recognised by the legislation it is more likely that they will be connected. The recent nature of this change may also explain the lack of research in this area as health and safety climate may not have been hypothesised to relate to WIM as it previously did not cover WIM.

Although there is little research investigating the relationship between health and safety climate and WIM, the theory of climate's influence on WIM, and recent changes to legislation suggest that health and safety climate will be related to WIM.

Hypothesis 3: Health and safety climate will be negatively related to WIM.

\section{Service Cultures}

It is hypothesised that certain organisational cultures may encourage WIM as part of their training and socialisation processes. Archer (1999) identified features of paramilitary culture which may contribute to WIM. Within the NZDF there are three services: Navy, Army and Air Force with three different histories and traditions. Therefore, it is possible that the nature of these different cultures may influence the incidents of WIM, even within the larger NZDF culture. The culture of an organisation is influenced by the work that the organisation conducts. A key difference in the way work is conducted across the services is the interaction between personnel and equipment. Both Navy and Air Force primarily deal with the operation of large pieces of technical equipment (ships and aircraft respectively). In contrast, in the Army the primary focus is on people and the performance of groups 
of soldiers (Groysberg, Hill, \& Johnson, 2010). The primarily interpersonal nature of Army work, with a high requirement for co-operation between individuals and groups may increase the likelihood of WIM (Einarsen, 2000).

Hypothesis 4: Army will demonstrate more WIM, compared with civilians, than either Navy or Air Force.

\section{Leadership Behaviour}

The content of leadership behaviour can demonstrate different styles of leadership. One distinction is between transactional (or directive) and transformational leadership. Directive leadership relies on exchange theory; leaders achieve outcomes by offering benefits in exchange for the subordinates time and effort. Power is gained by what the leader has to offer, and if the subordinate determines that they do not value what the leader is offering they may choose not to comply.

Transformational leadership relies on the ability of the leader to influence subordinates by conveying a vision and getting subordinates to commit to the vision.

\section{Directive Leadership}

As the supervisor is in a position of power in the workplace they are directly involved in creating an environment where WIM can or cannot occur, as well as sending messages about how the organisation views WIM when it does occur. Therefore, supervisor behaviour is an integral factor in enabling WIM.

Subordinate ratings of leadership behaviours such as providing instructions, guidance and feedback has been demonstrated as the organisational factor second most strongly related to bullying, after role conflict (Einarsen, Raknes, \& Matthiesen, 1994). The relationship between leadership performance and bullying was found to be consistent across different employee types such as teachers, trade and commerce workers and electricians (Einarsen, Raknes \& Matthiesen, 1994). In this study leadership behaviour was considered to be a feature of the work environment. Lack of task related feedback is also significantly related to bullying (Notelaers, De Witte \& Einarsen, 2010). Many of these behaviours can be considered features of directive or transactional leadership in that they represent performing the basic functions of 
leadership. Directive leadership has been defined as leadership which focused on implementing tasks through the enforcement of rules and procedures (Black, 1990).

The absence of these directive leadership behaviours can encourage WIM. The absence of even basic leadership behaviours has been called 'laissez-faire leadership'. Laissez-faire leaders abdicate their rights and responsibilities as leaders and allow subordinates to make their own decisions about how they behave (Kalliath, Brough, O’Drisocoll, Manimala \& Siu, 2010). Therefore, laissez-faire leadership is an alternate way of conceptualising the lack of directive leadership behaviour such as feedback and guidance. Laissez-faire leadership is also particularly important in WIM research as the abdication from leadership allows subordinates to develop their own behavioural norms which may include WIM.

Recent studies of leadership styles (particularly on laissez-faire leadership) have indicated leadership styles are related to WIM. Leadership style has been linked to experiencing and observing workplace bullying. Unpredictable leadership styles where leadership behaviour (e.g. punishment) is unrelated to subordinates behaviour were most strongly related to experienced self-reported bullying and autocratic leadership styles were most associated with bullying observed by others. A laissezfaire leadership style was related to experienced but not observed bullying in a representative sample of British workers (Hoel, Glaso, Hetland, Cooper \& Einarsen, 2010). Contrastingly, New Zealand researchers identified that laissez-faire leadership was related to both observed and experienced bullying and those personnel who reported both observing and experiencing bullying reported more laissez-faire leadership, than those who only observed or experienced bullying alone (CooperThomas, Catley, Bentley, Gardner, O’Driscoll \& Trenberth, 2011).

Other employee perceptions of leadership are also related to WIM. Perceived leadership fairness is also important: lower scores on fair leadership were associated with more bullying (Mageroy, Lau, Riise \& Moen, 2009). These findings suggest that the absence of important supervisory behaviours can either be viewed as WIM by subordinates or can allow an environment where WIM can occur, between coworkers, to develop. 
Existing research provides evidence for at least two mechanisms by which leadership is related to WIM. These mechanisms are by influencing the social norms of the group through the feedback and guidance they provide to subordinates, and through creating a stressful work environment which elicits WIM (Bowling \& Beehr, 2006). The performance of even basic directive leadership behaviours will reduce the occurrence of WIM.

Hypothesis 5: Directive leadership will be negatively related to WIM.

\section{Power}

Stress and tension or frustration at work may trigger the search for a scapegoat. A scapegoat is a person who does not have much power in a situation. They are a safe target for displaced aggression as they do not have the means to exact revenge on the aggressor (Einaresen, Hoel, Zapf \& Cooper, 2003). Any person who has less access to resources or influence in the organisation may become a scapegoat. Alternatively, low power individuals may be subjected to WIM by high power individuals in order to maintain the dominance of the existing culture. This was the case in a study of the fire-service by Archer (1999) where white males bullied females and non-whites as the presence of these demographic groups in the work environment threatened the existing white patriarchal culture. This can explain why certain groups report more WIM than others. Groups which are powerless in some way because they represent a minority and do not have power or access to resources in a situation are more easily bullied.

Power has been operationalised in WIM research as organisational position and in some industries as gender (Keashly \& Jagatic, 2003). Membership of another minority group such as a minority ethnic group is another way to operationalise power in organisations.

\section{Seniority}

Seniority in the organisation is hypothesised to protect people from WIM as more senior personnel are less accessible targets. Seniority can be measured by a range of 
proxies such as rank (for military samples), income or age. In many groups these three variables are likely to be correlated. The finding that seniority in an organisation is a protective factor for WIM has been consistently demonstrated across levels of measurement and industry groups. At a group level, a lower mean age at a departmental level was found to be associated with more bullying (Mageroy, Lau, Riise \& Moen, 2009). In the military, lower rank was found by Fitzgerald, Drasgow, Magley, and Waldo (1999) to be related to increased unwanted sexual attention. Enlisted (lower ranked) female personnel reported more cases of unwanted sexual attention (especially sexual coercion type behaviours) than female officers (who hold higher rank). Age has also been demonstrated to be related to WIM. Ostvik and Rudmin (2001) found that younger personnel in the Norwegian Army were most at risk of bullying.

Hypothesis 6: Seniority will be negatively related to WIM.

\section{Gender}

Across 27 studies summarised by Zapf, Einarsen, Hoel and Vartia (2003) women are reported to be the victims of WIM approximately twice as often as men. This is thought to be because women are less likely to hold positions of power and are therefore easier targets of WIM. In addition, due to society's gendered expectations of female behaviour as caring and sensitive, women may be less able to defend themselves assertively against WIM once it has begun. Researchers have demonstrated empirically gender differences in sexual harassment (e.g. Gutek, 1995).

Especially in male-dominated industries, women report more WIM than men (McCarthy \& Mayhew, 2004). This finding has been replicated in the military, where women report more harassment than men (Donovan \& Drasgow, 1999).

Hypothesis 7: Females will report more WIM compared to males.

\section{Ethnicity}

Ethnic minorities report more WIM than ethnic majorities, and attribute this perceived discrimination (behaviour such as "people act as if you are inferior") most commonly to their race or ethnicity over other individual characteristics such as 
appearance (Kessler, Mickelson \& Williams, 1999; Pavalko, Mossakowski and Hamilton, 2003). In military samples, significant ethnic differences have been found in experiences of discrimination. Scarville, Button, Edwards, Lancaster and Elig (1999) report 10-18\% of ethnic minorities experiencing discriminatory behaviours that impact their careers, compared to $4 \%$ for whites.

Hypothesis 8: Ethnic minorities will be report more WIM compared to the ethnic majority (NZ Europeans).

\section{Consequences}

\section{Emotional States}

\section{Stress}

Although stress is a commonly studied outcome of WIM, not much research has been conducted into the reasons for this relationship (Einarsen \& Mikkelsen, 2003). However, two possible theories exist for why stress occurs as a result of WIM (Einarsen \& Mikkelsen, 2003). The first is related to schemas and expectations. All people hold schemas which describe their expectations of the world and their role in the world. Such schemas may include content such as 'I am a good worker' and 'I am a valued member of the team'. These schemas allow people to function socially as they reduce cognitive load and uncertainty. The content also tends to be positive. When WIM occurs it can undermine these schemas and expectations and cause a sense of loss and vulnerability for people. The individual finds the experience very threatening and therefore stressful. The second mechanism is socio-biological in nature and relates to social exclusion. Social exclusion or ostracism causes stress because of the basic social nature of humans (Williams, 1997). Exposure to ostracism violates basic human needs such as: a sense of belonging to others, a sense of selfworth or esteem by being deemed unworthy of others care and respect, a need for control over one's environment. The violation of these basic needs causes pain, anxiety and worry (Einarsen \& Mikkelsen, 2003). Even such innocuous social exclusion as having two people acting as participants in an experiment withdraw from including a third person in a game of 'catch' can bring on strong feelings of stress (Williams, 1997). 
Stress is an individual level outcome, which has work-related effects. Willness, Steel and Lee (2007) found sexual harassment to have a negative impact on victims' mental health including displaying symptoms of post-traumatic stress disorder. In a multidimensional model of workplace bullying, distress at work was found to be consequences of workplace bullying in Australian nurses (Hutchinson, Wilkes, Jackson \& Vickers, 2010). Psychological distress has also been identified as an outcome of harassment in work by Nye, Brummel and Drasgow (2009). Additionally, work in NZ military deployed populations has demonstrated that WIM is moderately correlated with psychological distress in deployed New Zealand military populations (Kearney, 2009).

Hypothesis 9: WIM will be positively related to stress.

\section{Attitudes}

\section{Job Satisfaction}

The decrease in job satisfaction is one of the most consistently studied organisational outcomes of WIM. Job satisfaction is hypothesised to decrease as a result of WIM due to the importance of quality human interactions in enjoyment of one's job (Einarsen, Raknes \& Matthiesen, 1994). Meta-analytic techniques have identified decreased job satisfaction as consequences of sexual harassment (Willness, Steel \& Lee, 2007). Sexual harassment has a greater impact on interpersonal satisfaction (with co-workers and supervisors) than work-type satisfaction. Work-type satisfaction was reduced to a similar degree to global satisfaction. Also using meta-analytic techniques, Lapierre, Spector, and Leck (2005) established that sexual workplace aggression significantly reduces global job satisfaction.

In military samples work satisfaction was more negatively influenced by sexual harassment than in non-military samples. This is perhaps due to the intertwining of interpersonal and work life in the military (Willness, Steel \& Lee, 2007). For service personnel their work, social, sporting, and family lives and even their health are all part of military service. This is evidenced by the provision of services in these areas by the organisation. The provision of messes (military bars) by the organisation supports social interaction. The provision of opportunities to participate in military- 
coordinated sporting competitions, and physical training in work time, results in sporting and social life becoming part of work. The provision of military health and dental services also means that an individual's health is linked with their work.

Extending beyond sexual harassment, Lim and Cortina (2005) found that forms of workplace disrespect were linked with sexual and gender harassment and were associated with job satisfaction, and job stress. Bowling and Beehr (2006) used metaanalytic techniques to demonstrate that WIM accounts for significant variance in job satisfaction. The negative effects of WIM on job satisfaction have been demonstrated across cultures. Job satisfaction and workgroup identification were found to be negatively related to bullying, in both Australian and Singaporean employees, although the relationships were more pronounced in Australia (Loh, Restubog, \& Zagenczyk, 2010).

In the discrimination literature, perceived discrimination has been found to be related to lower job satisfaction (Ensher, Grant-Vallone, \& Donaldson, 2001).

The consistency of the finding of the decrease of job satisfaction in response to WIM leads to hypothesis 10 .

Hypothesis 10: WIM will be negatively related to job satisfaction.

\section{Organisational Commitment}

Organisational commitment is another commonly studied outcome of WIM.

Organisational commitment refers to a organisational member who "identifies with, is involved in, and enjoys membership in, the organization" (Allen \& Meyer, 1990, p2). Organisational commitment decreases in response to WIM as a result of the target attributing WIM to the organisation, at least in part (Bowling \& Beehr, 2006). Decreased organisational commitment has been evidenced in three important WIM domains: harassment, discrimination and bullying.

First, lowered organisational commitment and withdrawal from work have been identified as consequences of sexual harassment using meta-analytic techniques (Willness, Steel \& Lee, 2007). Second, Yildirim (2009) conducted a study of female Turkish nurses using a behavioural measure of workplace bullying and found that they reported lowered work motivation, poor organisational commitment, and poor 
relationships with people at work in response to workplace bullying. Third, perceived discrimination has been found to be related to lower organisational commitment and organisational citizenship behaviours (Ensher, Grant-Vallone, \& Donaldson, 2001). Therefore, WIM is hypothesised to predict organisational commitment.

Hypothesis 11: WIM will be negatively related to organisational commitment.

\section{Leaving Intentions}

Leaving intentions or leaving behaviour is a distal outcome of WIM. Victims of WIM may show increased leaving intentions for a number of reasons; either due to reciprocity: the desire to get even with the organisation by leaving and taking their knowledge and skills with them or as a means to escape the situation and reduce stress (Hoel, Einarsen \& Cooper, 2003).

The effect of WIM on leaving behaviour has been demonstrated longitudinally by Sims, Drasgow and Fitzgerald (2005) who found that sexual harassment was linked to increased turn over for females in the military, even after controlling for job satisfaction and organisational commitment. Exit behaviour such as preparing a $\mathrm{CV}$ has been linked with exposure to sexist behaviour and gender discrimination (Nye, Brummel \& Drasgow, 2009).

Leaving intentions or job withdrawal is often used in the absence of actual leaving behaviour data. Bowling and Beehr (2006) found that reported WIM predicted turnover intentions. Lim and Cortina (2005) found sexual and gender harassment were related to job withdrawal. This relationship also extends to witnesses of WIM. Rayner (1999) reported 20\% of witnesses of workplace bullying considered leaving the organisation due to observing bullying taking place. In this thesis, WIM is therefore expected to predict leaving intentions.

Hypothesis 12: WIM will be positively related to leaving intentions. 


\section{Summary}

This thesis fills an important gap in the literature by addressing the nature of workplace interpersonal mistreatment as well as organisationally important antecedents and consequences. The approach used is novel in this field because it uses a large sample of existing data from a practitioner-developed tool to address the research questions. The use of military data is particularly interesting because it gives a unique perspective into WIM in a military environment. The outcomes of this thesis make an important contribution to the study of WIM in organisations. 


\begin{abstract}
APPROACH
This thesis has two broad aims: 1) to identify constructs measured by a practitioner developed survey; and 2) to test a model of the antecedents and consequences of WIM in this data. In order to address these two aims multiple studies will be conducted. The way that these studies proceed follows the 'researcher-inherit' model and accepted practice for structural equation modelling (SEM).
\end{abstract}

\title{
Researcher-Inherit Model
}

When working with researcher-inherit data, Robert Vandenberg advocates beginning with Exploratory Factor Analysis (EFA) procedures to identify logical groupings of items. However, it should be noted that even these logical groupings of items are a post hoc exercise, which must occur prior to undertaking the main analyses to answer the study's hypotheses (Hurley, et al., 1997). In accordance with this recommendation the first step in this thesis is to begin with EFA procedures to identify logical groupings of items, and then validate the groupings of items using confirmatory factor analysis (CFA). The movement through exploratory and confirmatory phases is also a feature of sequential SEM procedures.

\section{Sequential SEM}

SEM techniques are the only type of analysis that allows the research to simultaneously and completely test all of the relationships in complex and multidimensional phenomena (Tabachnick \& Fidell, 2007). SEM encompasses the benefits of factor analysis and regression in that it allows measurement error associated with the measurement of latent constructs to be partitioned from the relationships between those latent constructs, something which regression techniques or path models alone cannot accomplish (Tabachnick \& Fidell, 2007). However, prior to testing the relationships between constructs, the adequacy of the measurement model must first be demonstrated empirically. This is particularly important when using measures which do not have established construct validity. When developing this approach, Joreskog (1993) noted that validating the 
measurement model is an important step that must be conducted prior to testing the structural model:

the testing of the structural model - that is, testing of the initially specified theory may be meaningless unless it is first established that the measurement model holds. If the chosen indicators for a construct do not measure that construct, the specified theory must be modified before it can be tested.' ( $\mathrm{p} 297$ ).

Additionally, the use of calibration and validation samples is critical in establishing the measurement model separate from the structural model (Joreskog, 1993). If only one sample is used for the entire process the model is unlikely to generalise to the population as the data analysis strategy will not have controlled for the capitalisation on chance:

"Replication substantially enhances the scientific contribution of any factor-analytic work, so that researchers might want to consider using EF $A$ and $C F A$ in multi-sample studies, perhaps first exploring and then confirming one or more particular structural bypotheses." (Hurley et al., 1997; p 673)

This thesis uses the recursive method where exploratory and hypothesis generating phases are cycled through more than once from the researcher-inherit literature and the analysis strategy described by Joreskog (1993) used in the SEM literature.

\section{Studies}

The research process is detailed in figure 4. Multiple studies were conducted to meet the two aims of this thesis. In order to evaluate the psychometric properties of the practitioner-developed questionnaire, principle component and confirmatory factor analysis were conducted using different samples. This phase of the research develops and then confirms the measurement model. This addresses the first aim and is detailed in study one. In order to develop and validate the 12 hypotheses outlined above, regression followed by structural equation modelling was conducted in three different samples. This phase of research developed and confirmed the structural model, addressing the second broad aim. The results are detailed in studies two and three. Arrows in figure 4 communicate the way that the results of the earlier studies 
inform testing in subsequent studies. For example the results of the PCA conducted on sample one inform both the regression of antecedents onto WIM and the confirmatory factor analysis conducted on sample two.

Figure 4: Approach to research using multiple samples

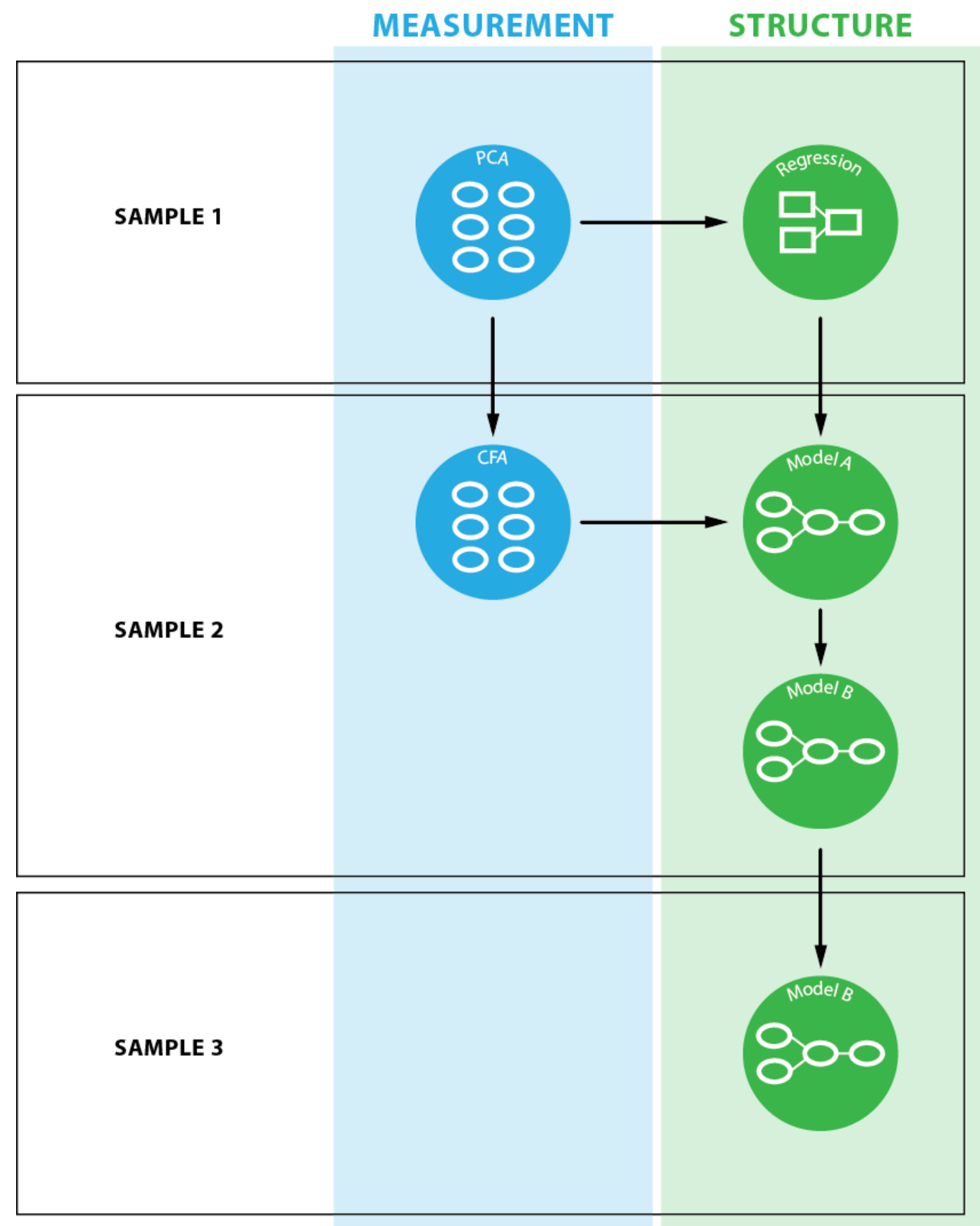




\section{METHOD}

\section{Data}

The data used in this survey comes from the New Zealand Defence Force's Ongoing Attitude Survey (OAtS). The OAtS survey was administered by the NZDF

Directorate of Psychology. It is a survey of attitudes and perceptions of employment in the NZDF. The OAtS has 206 items in fifteen sections:

1. Organisational commitment;

2. Job satisfaction;

3. Organisational culture;

4. Work/life balance;

5. Health and safety;

6. Equity;

7. Supervision;

8. Senior leadership;

9. Performance management;

10. Career development;

11. Training and resources;

12. Change;

13. Career intentions;

14. Morale-satisfaction and Conditions of service; and

15. General. 
The number of items in each section varies from eight to 23. Responses are anchored on a 5 -point scale where 1 corresponds to strongly disagree and 5 corresponds to strongly agree. A copy of the NZDF OAtS questionnaire is included in Appendix A.

\section{Origins of the Ongoing Attitude Survey}

The sections of the survey are not based on published literature except for some items in the organisational commitment section. Instead, the items originated from a number of sources. Some items are used for benchmarking with New Zealand organisations and these originate from the JRA Best Places to Work (BPTW) survey sponsored by unlimited magazine and conducted annually within New Zealand. Additionally, some items are used for benchmarking with other nations' militaries and were negotiated through The Technical Co-operation Panel's HUM-TP3 group ${ }^{1}$. The remaining items were based on Australian Defence Force (ADF) continuous attitude survey and discussions with organisational stakeholders such as the NZDF EEO and Equity Unit. The current version of the NZDF OAtS questionnaire has been in use since July 2007.

\section{Demographic and Employment Data}

Additional data is matched to the OAtS responses from the NZDF HR system using respondents' service numbers. The matched data includes demographic information such as date of birth, gender, and marital and family status; as well as employment information such as length of employment, rank, branch, most recent pay and current posting information.

\section{Participants}

The questionnaire is administered to NZDF regular force (RF, full time) and civilian personnel. Territorial force (TF, part time) personnel are not invited to participate. Additional personnel that are excluded from sampling include: personnel with fewer than 3 months service or with a MD717 (voluntary release) in process, personnel on leave, and anyone who has completed the OAtS in the previous year. From this target population, a randomly selected $1 / 12^{\text {th }}$ is then invited to complete the OAtS.

${ }^{1}$ The TTCP group includes Australia, Canada, New Zealand, the United Kingdom, and the United States. 
The intent of this system is to request all NZDF RF and civilian personnel complete the NZDF OAtS questionnaire no more than once per year (Sarah Overdale, personal communication, 31 November, 2009). Responses are grouped into quarters by date of completion (as there is no time limit on responding). For the following analysis units with data from less than 5 participants were excluded from analysis to allow for future group level comparison and multi-level modelling which are planned for this dataset (resulting in 167 cases excluded). This approach is common practice in multilevel research (e.g., Gilson, Mathieu, Shalley, \& Ruddy, 2005; Glomb \& Liao, 2003).

\section{Data Cleaning}

The total data set used in this thesis includes data from 2007, 2008 and 2009 with the following dataset is available for analysis.

Table 2: Number of participants in each of the three datasets

\begin{tabular}{lcc}
\hline Year & Frequency & Percent \\
\hline 2007 & 2312 & 21.6 \\
2008 & 4722 & 44.1 \\
2009 & 3663 & 34.2 \\
Total & 10697 & 100.0 \\
\hline
\end{tabular}

The largest dataset 2008 (sample 1) is used for the initial PCA in order to identify groupings of items which have a high likelihood of generalising to the other samples and the population (McCall \& Appelbaum, 1991). The other samples (2007, sample 2, and 2009, sample 3) form the validation samples, and are available for confirmatory factor analysis and SEM modelling (Jöreskog, 1993; Gerbing \& Hamilton, 1996).

\section{Missing Data}

Tabachnick and Fidell report that the pattern of missing data is of more concern than the amount and that missing data 'is one of the most pervasive problems in data analysis (Tabachnick \& Fidell, 2007, p 62). If data is missing 'not-at-random' it can affect how well the results of any analysis will generalise. Items not missing at random were due to two reasons: 
1. items have been introduced or removed over time resulting in missing data across the 3 years of data used in this thesis,

2. items do not apply to everyone (n/a responses) and inclusion would result in data from specific groups being excluded from subsequent analyses using listwise deletion.

Items that showed data not missing at random were excluded from the analysis. Additionally, one section of the OAtS (senior leaders section) contains items which have a lot of missing data that appeared to be missing randomly; those items which have more than 5\% missing were excluded. The 5\% threshold for missing data is applied for the whole sample (years 2007, 2008 and 2009). One possible reason for this data to be missing is that it refers to communication between senior leaders and personnel and many personnel may not have had the opportunity to communicate directly with senior leaders. The removal of the items listed above from analysis leaves 130 items for use in analysis. 


\section{STUDY 1: MEASUREMENT MODEL}

This thesis aims to identify the constructs relevant to WIM that are measured by the NZDF Ongoing Attitude Survey. Principle component analytic and confirmatory factor analytic techniques were used with two different samples of data to explore and then confirm the measurement model.

\section{Analysis}

\section{Principle Component Analysis}

Parallel analysis is a technique used to determine the number of factors to retain in a given factor structure (O'Connor, 2000). It is a threshold based method where one can identify the number of components in a structure which account for more variance than the components derived from random data. It essentially identifies the 'important' factors. It is considered more robust than examination of the scree plot or using the threshold of eigenvalues greater than 1 (O'Connor, 2000).

Random data were generated based on features of the OAtS dataset including the number of variables and cases, and features determined by the researcher; the number of random datasets to generate. A PCA was then conducted on the random data. The mean eigenvalues from the (in this case 100) random datasets was compared to the eigenvalues from the OAtS data in order to determine which factors in the OAtS dataset explain more variance than those derived from random data.

Following the parallel analysis a principle components analysis was conducted with the solution forced to number of factors that the parallel analysis identified. The principle component analysis method of extraction of factors was chosen due to the exploratory nature of this thesis, with a promax rotation to account for the likely correlation between factors (Field, 2005). Stevens (1992) suggests that for sample sizes of 100 or greater, factor loadings of greater than 0.162 can be important. Therefore the pattern matrix was set to display factor loadings above 0.1 as the sample was much greater than 100 .

Principle components analysis was conducted using SPSS 18, on a sample of 4722. 


\section{Confirmatory Factor Analysis}

Confirmatory factor analytic techniques were then used on a second sample (sample 2: data from 2007) to confirm the structure of factors obtained in the PCA. Only factors which had theoretical or empirical relevance to WIM in the existing literature were analysed using CFA.

A CFA was conducted using AMOS 18 and missing data was deleted listwise, leaving a sample of 2082 participants. Each relevant construct is analysed in turn, except for constructs which have only three indicators. Constructs with only three indicators are analysed concurrently with another construct due to the limitations of AMOS 18. Where the initial solution for each construct showed inadequate fit, further analysis evaluated each item's conceptual and statistical contribution to the construct. Items which did not contribute on either of these grounds were removed.

\section{Statistics Used to Evaluate Model Fit}

A range of goodness-of-fit indices are used to evaluate model fit, based on the recommendations by Tanaka (1993). Four fit indices: CFI, TLI, RMSEA and SRMR are used.

The CFI (comparative fit index) is truncated to fall in the range from 0 to 1 . CFI values close to 1 indicate a very good fit (Bentler, 1990). CFI is one of the most commonly reported fit measures.

The typical range for TLI (Tucker-Lewis Coefficient or Bentler-Bonett non-normed fit index; NNFI) lies between zero and one, but it is not limited to that range. TLI values close to 1 indicate a very good fit (Bentler \& Bonett, 1980). Both CFI and TLI are normed indices which are more commonly preferred in the behaviour sciences as they can be used to make comparisons of models across studies using different data (Tanaka, 1993). TLI also favours simple models and will penalise models which are too complex (over parameterised, Tanaka, 1993). The inclusion of a measure which controls for model complexity constrains the solution to theoretically and practically useful models. As the samples used in this thesis are very large, the use of a fit index which is independent (TLI) of sample size is advised (Tanaka, 1993). Tanaka (1993) advises that with very large samples achieving model fit is more difficult as with large 
samples it will be more likely that the discrepancy between the model and the data will be statistically significant. Therefore, in this thesis less attention has been paid to the chi square significance test, and more weight is placed on other fit indices such as TLI.

Browne and Cudeck (1993) recommend an RMSEA (root mean square error of approximation) of approximately .05 or less would indicate a close fit of the model in relation to the degrees of freedom. The authors recommend the use of .05 over a value of 0 which would indicate exact fit but which may be unreasonable. At the upper end a value of 0.08 or less indicates a reasonable error of approximation. The authors place an upper limit of 0.1 for the RMSEA.

The SRMR (standardized RMR, root mean square residual) is an absolute measure of fit and is defined as the standardized difference between the observed correlation and the predicted correlation. The smaller the SRMR, the better: a value of zero indicates perfect fit and a value less than .08 is generally considered a good fit. The SRMR is an absolute measure of fit and is a positively biased measure. This means the SRMR tends to be smaller as sample size increases and as the number of parameters in the model increases. As the sample size in this thesis is large, the SRMR is likely to be biased towards smaller values. The SRMR has no penalty for model complexity $(\mathrm{Hu}$ \& Bentler, 1999). 


\section{Results}

\section{Summary Statistics}

Table 3: Descriptive statistics and correlation among the study variables for sample 1

\begin{tabular}{|c|c|c|c|c|c|c|c|c|c|c|c|}
\hline & & $\mathrm{M}$ & SD & 1 & 2 & 3 & 4 & 5 & 6 & 7 & 8 \\
\hline 1 & Directive Leadership & 3.8315 & 0.71099 & - & & & & & & & \\
\hline 2 & Organisational Commitment & 3.9910 & 0.55406 & $.333 * *$ & - & & & & & & \\
\hline 3 & Aligned-Cohesive Culture & 3.5205 & 0.62163 & $.391 * *$ & $.653 * *$ & - & & & & & \\
\hline 4 & WIM & 2.1661 & 0.86907 & $-.302 * *$ & $-.258 * *$ & $-.283 * *$ & - & & & & \\
\hline 5 & Job Satisfaction & 3.6216 & 0.76967 & $.358^{* *}$ & $.543 * *$ & $.451^{* *}$ & $-.228 * *$ & - & & & \\
\hline 6 & Leaving Intentions & 2.7005 & 1.00789 & $-.290 * *$ & $-.504 * *$ & $-.482 * *$ & $.246^{* *}$ & $-.450^{* *}$ & - & & \\
\hline 7 & Health and Safety & 4.0034 & 0.53586 & $.366^{* *}$ & $.349 * *$ & $.339 * *$ & $-.245^{* *}$ & $.290 * *$ & $-.189 * *$ & - & \\
\hline 8 & Stress & 2.5183 & 0.81211 & $-.223 * *$ & $-.207 * *$ & $-.284 * *$ & $.311 * *$ & $-.199 * *$ & $.348^{* *}$ & $-.192 * *$ & - \\
\hline 9 & Work Life Balance & 3.4269 & 0.62103 & $.190 * *$ & $.273 * *$ & $.381 * *$ & $-.221 * *$ & $.208^{* *}$ & $-.335^{* *}$ & $.167 * *$ & $-.490 * *$ \\
\hline 10 & Equity and Diversity Climate & 3.6309 & 0.63064 & $.229 * *$ & $.398^{* *}$ & $.390 * *$ & $-.316^{* *}$ & $.278^{* *}$ & $-.223^{* *}$ & $.382^{* *}$ & $-.148^{* *}$ \\
\hline 11 & Attitude to Change & 2.5620 & 0.58929 & $-.201 * *$ & $-.363 * *$ & $-.451 * *$ & $.169 * *$ & $-.204 * *$ & $.310^{* *}$ & $-.201 * *$ & $.242^{* *}$ \\
\hline 12 & Pay Fairness & 2.7314 & 1.02591 & $.128 * *$ & $.262^{* *}$ & $.355^{* *}$ & $-.126^{* *}$ & $.160^{* *}$ & $-.299 * *$ & $.081 * *$ & $-.168 * *$ \\
\hline 13 & Promotion & 3.4873 & 0.78046 & $.346^{* *}$ & $.524 * *$ & $.526^{* *}$ & $-.187 * *$ & $.427 * *$ & $-.470 * *$ & $.266^{* *}$ & $-.197 * *$ \\
\hline 14 & Work Quality Standards & 4.3237 & 0.49879 & $.207 * *$ & $.421 * *$ & $.214^{* *}$ & $-.144^{* *}$ & $.426 * *$ & $-.167 * *$ & $.318^{* *}$ & $-.119 * *$ \\
\hline 15 & Skills and Training & 3.6554 & 0.64785 & $.415^{* *}$ & $.383^{* *}$ & $.503 * *$ & $-.244 * *$ & $.372^{* *}$ & $-.332^{* *}$ & $.372^{* *}$ & $-.247 * *$ \\
\hline 16 & Performance Management & 3.3051 & 0.91806 & $.463 * *$ & $.272^{* *}$ & $.394 * *$ & $-.200 * *$ & $.289 * *$ & $-.290 * *$ & $.224 * *$ & $-.194 * *$ \\
\hline 17 & Contribution & 3.9519 & 0.58915 & $.276^{* *}$ & $.577 * *$ & $.463 * *$ & $-.204 * *$ & $.454 * *$ & $-.271 * *$ & $.364 * *$ & $-.123^{* *}$ \\
\hline 18 & Employment Opportunities & 3.6371 & 0.83585 & $-.030^{*}$ & $-.054 * *$ & $-.156^{* *}$ & $.050^{* *}$ & $-.042^{* *}$ & $.233^{* *}$ & $.051^{* *}$ & $.094 * *$ \\
\hline 19 & Resourcing & 3.1578 & 0.85796 & $-.085^{* *}$ & $-.155^{* *}$ & $-.317 * *$ & $.070^{* *}$ & $-.128^{* *}$ & $.180^{* *}$ & $-.071 * *$ & $.156^{* *}$ \\
\hline
\end{tabular}

**. Correlation is significant at the 0.01 level (2-tailed). *. Correlation is significant at the 0.05 level (2-tailed). 


\begin{tabular}{|c|c|c|c|c|c|c|c|c|c|c|c|}
\hline & & 9 & 10 & 11 & 12 & 13 & 14 & 15 & 16 & 17 & 18 \\
\hline 1 & Directive Leadership & & & & & & & & & & \\
\hline 2 & Organisational Commitment & & & & & & & & & & \\
\hline 3 & Aligned-Cohesive Culture & & & & & & & & & & \\
\hline 4 & WIM & & & & & & & & & & \\
\hline 5 & Job Satisfaction & & & & & & & & & & \\
\hline 6 & Leaving Intentions & & & & & & & & & & \\
\hline 7 & Health and Safety & & & & & & & & & & \\
\hline 8 & Stress & & & & & & & & & & \\
\hline 9 & Work Life Balance & - & & & & & & & & & \\
\hline 10 & Equity and Diversity Climate & $.154^{* *}$ & - & & & & & & & & \\
\hline 11 & Attitude to Change & $-.233^{* *}$ & $-.233^{* *}$ & - & & & & & & & \\
\hline 12 & Pay Fairness & $.277^{* *}$ & $.189 * *$ & $-.182^{* *}$ & - & & & & & & \\
\hline 13 & Promotion & $.191 * *$ & $.306^{* *}$ & $-.368^{* *}$ & $.272^{* *}$ & - & & & & & \\
\hline 14 & Work Quality Standards & $.099 * *$ & $.206^{* *}$ & $-.127 * *$ & $-.085^{* *}$ & $.159 * *$ & - & & & & \\
\hline 15 & Skills and Training & $.297 * *$ & $.263^{* *}$ & $-.290^{* *}$ & $.193^{* *}$ & $.415^{* *}$ & $.180^{* *}$ & - & & & \\
\hline 16 & Performance Management & $.201 * *$ & $.206^{* *}$ & $-.196^{* *}$ & $.202^{* *}$ & $.368^{* *}$ & $.088^{* *}$ & $.351^{* *}$ & - & & \\
\hline 17 & Contribution & $.151 * *$ & $.371 * *$ & $-.259 * *$ & $.121 * *$ & $.342^{* *}$ & $.394 * *$ & $.292^{* *}$ & $.197 * *$ & - & \\
\hline 18 & Employment Opportunities & $-.148^{* *}$ & .016 & $.035^{*}$ & $-.236 * *$ & $-.057 * *$ & $.138^{* *}$ & $-.080^{* *}$ & $-.063^{* *}$ & $.055^{* *}$ & - \\
\hline 19 & Resourcing & $-.234 * *$ & $-.060^{* *}$ & $.211 * *$ & $-.184 * *$ & $-.138^{* *}$ & $-.033^{*}$ & $-.284 * *$ & $-.120 * *$ & $-.102 * *$ & $.129 * *$ \\
\hline
\end{tabular}


Table 4: Descriptive statistics and correlation among the study variables for sample 2

\begin{tabular}{|c|c|c|c|c|c|c|c|c|c|c|c|c|}
\hline & & M & SD & 1 & 2 & 3 & 4 & 5 & 6 & 7 & 8 & 9 \\
\hline 1 & Directive Leadership & 3.7430 & 0.71915 & - & & & & & & & & \\
\hline 2 & Trust in Supervisor & 3.9222 & 0.88916 & $.782^{* *}$ & - & & & & & & & \\
\hline 3 & Organisational Commitment & 3.9999 & 0.53893 & $.285^{* *}$ & $.270^{* *}$ & - & & & & & & \\
\hline 4 & Aligned-Cohesive Culture & 3.4673 & 0.59667 & $.344^{* *}$ & $.283^{* *}$ & $.592^{* *}$ & - & & & & & \\
\hline 5 & WIM & 2.1454 & 0.86274 & $-.309 * *$ & $-.262^{* *}$ & $-.266^{* *}$ & $-.300 * *$ & - & & & & \\
\hline 6 & Job Satisfaction & 3.7308 & 0.68916 & $.332^{* *}$ & $.318^{* *}$ & $.578^{* *}$ & $.446^{* *}$ & $-.275^{* *}$ & - & & & \\
\hline 7 & Leaving Intentions & 2.8159 & 1.06199 & $-.199 * *$ & $-.207 * *$ & $-.446^{* *}$ & $-.429 * *$ & $.254^{* *}$ & $-.457 * *$ & - & & \\
\hline 8 & Health and Safety & 4.0491 & 0.51763 & $.377^{* *}$ & $.286^{* *}$ & $.366^{* *}$ & $.356^{* *}$ & $-.334 * *$ & $.309^{* *}$ & $-.192^{* *}$ & - & \\
\hline 9 & Stress & 2.6000 & 0.83700 & $-.215^{* *}$ & $-.183^{* *}$ & $-.197 * *$ & $-.270^{* *}$ & $.318^{* *}$ & $-.258^{* *}$ & $.314^{* *}$ & $-.237 * *$ & - \\
\hline 10 & Equity and Diversity Climate & 3.6541 & 0.63092 & $.244 * *$ & $.213^{* *}$ & $.368 * *$ & $.356^{* *}$ & $-.344 * *$ & $.257 * *$ & $-.191 * *$ & $.371 * *$ & $-.120 * *$ \\
\hline
\end{tabular}

** Correlation is significant at the 0.01 level (2-tailed).

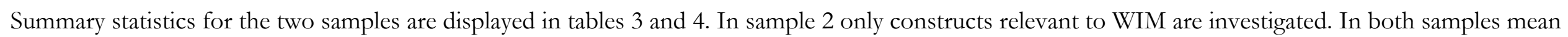

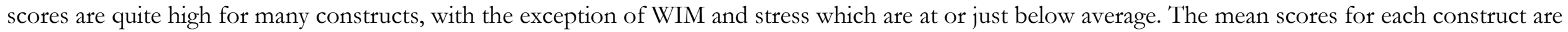

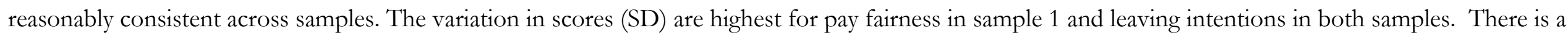

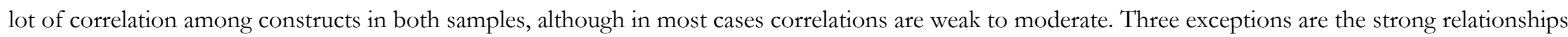

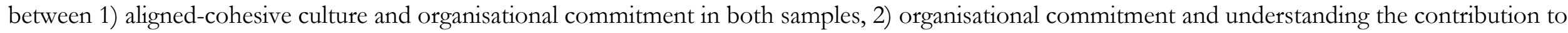
organisational goals in sample 1, and 3) directive leadership and trust in supervisor in sample 2. 


\section{Identifying the Number of Factors to Retain}

Random data eigenvalues from the parallel analysis were plotted against real sample eignevalues for sample 1 to identify the number of factors to retain in the PCA.

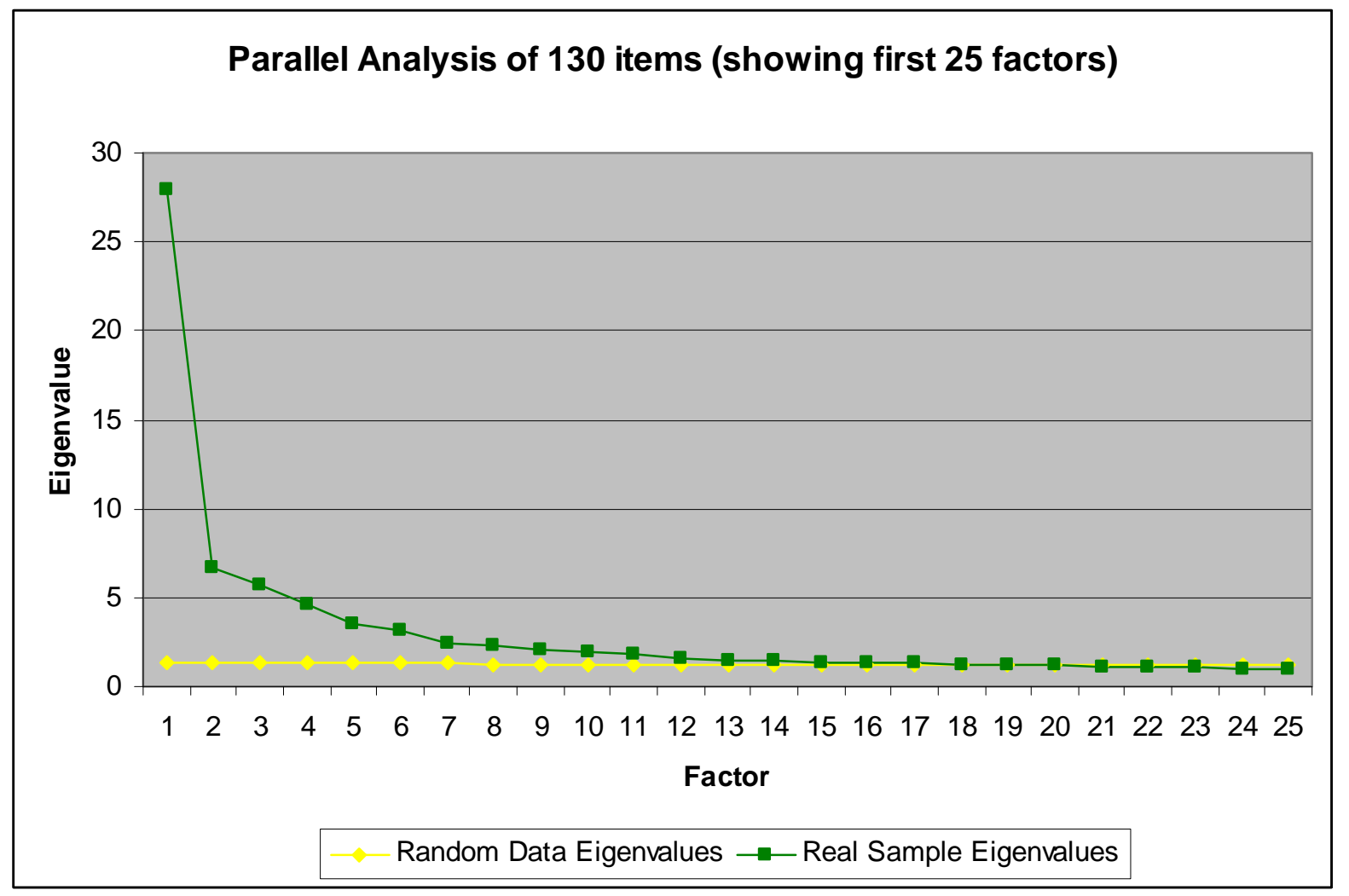

Figure 5: Parallel analysis to identify number of factors for retention

The plot above indicates that 19 factors should be retained in the PCA as this is where the OAtS sample eigenvalues line crosses the random data eigenvalues line (see close up in figure 6). The PCA solution was therefore constrained to 19 factors. 


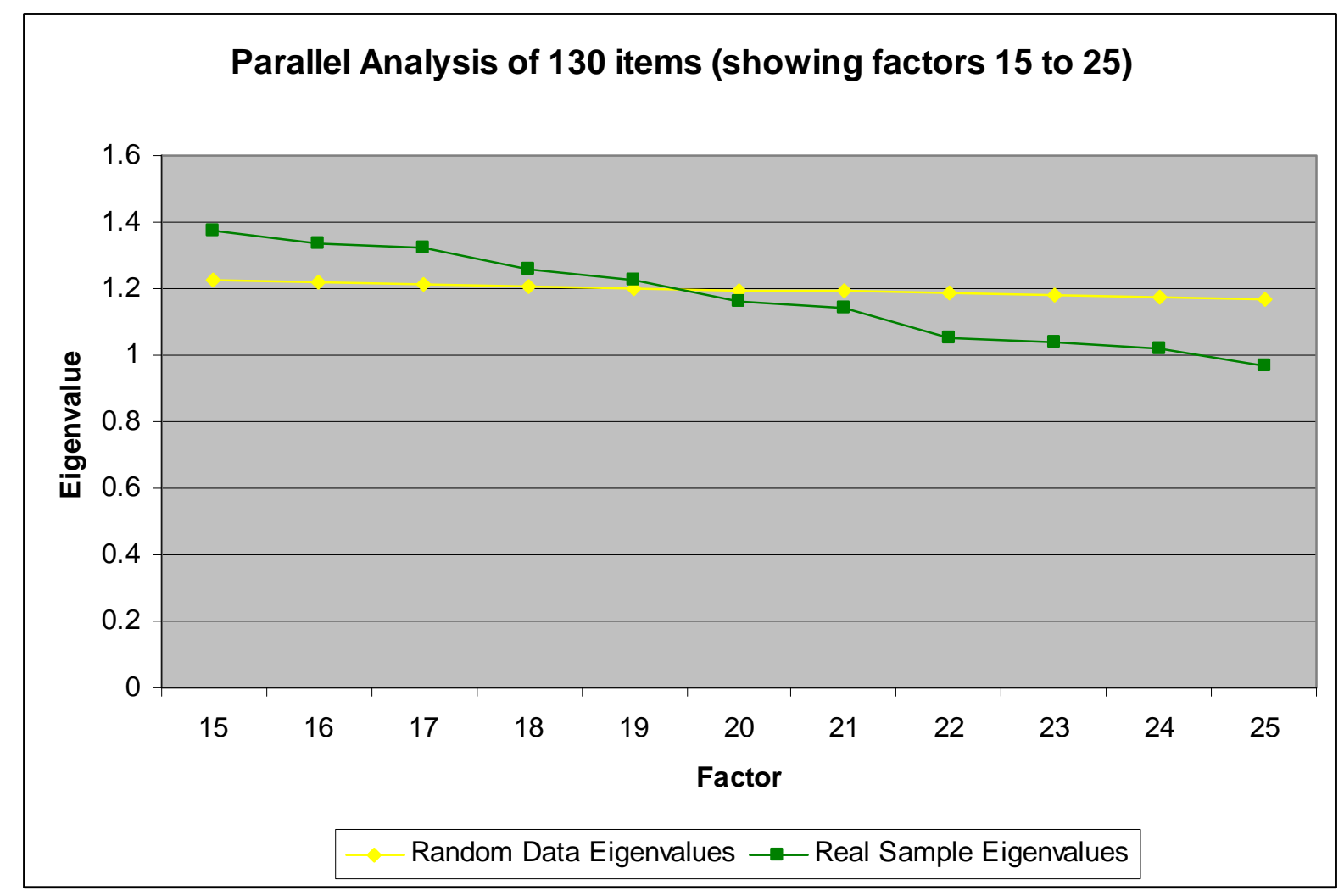

Figure 6: Close up of parallel analysis to identify number of factors for retention

\section{Items Contributing to Each Factor}

Results of the PCA indicated that a number of items in the solution cross loaded on more than one factor. Items which cross loaded on two or more factors with a difference of less than 0.2 were excluded from analysis as they did not discriminate sufficiently between factors. Further items were removed due to poor contribution to reliability (Cronbach's alpha). An exception to this procedure occurred for the $12^{\text {th }}$ Factor 'pay fairness' which has 3 items and an $\alpha$ of .878. The third item: 'My pay is comparable to what I would get outside the organisation', if deleted, would increase the $\alpha$ by .001, but would reduce the factor to a two item factor (which is not desirable). Due to the very small increase in reliability achieved by the deletion and the reasonably high factor loading for this item, it remained in this factor. The pool of 130 items that were included in the PCA was reduced to 90 items and the PCA identified the 19 factors detailed in table 5. All factors show good reliability except for Factors 18: 'Alternate Employment Opportunities', and 19 'Resources' which will 
not be included in any modelling as they are not theoretically related to WIM and showed poor structure.

\section{Confirmatory Factor Analysis}

Confirmatory factor analysis on the 9 constructs deemed theoretically relevant to WIM indicated that 4 showed inadequate fit when the solution found in the PCA was tested. These constructs were: WIM, directive leadership, aligned-cohesive culture, and leaving intentions. The initial and final fit statistics for each of these constructs are displayed in table 6 . In table 5 only the final loadings are displayed. Explanations for the changes made to these constructs are provided in the discussion, but are briefly summarised in the 'Implications' column in table 5 .

Table 5 indicates the item loading from the PCA in sample 1 and the CFA in sample 2. In addition, the section of the OAtS from which the originated from is displayed.

Table 6 indicates the fit statistics for each construct in the two samples. In sample 1 Cronbach's $\alpha$ is reported. In sample 2 Cronbach's $\alpha$ and the degrees of freedom, chi square, RMSEA, TLI, CFI, and SRMR. In the CFA, four constructs which showed poor fit and were respecified. For these four constructs CFA fit statistics for an initial solution and a final solution are displayed. In table 5 construct names given in italics are not relevant for the further studies. 
Table 5: Item loadings for each factor in the PCA and CFA

\begin{tabular}{|c|c|c|c|c|}
\hline & & PCA (Sample 1) & & CFA (Sample 2) \\
\hline Factor 1: Directive Leadership & OAtS Section & Factor loading & $\lambda^{2}$ & Implication \\
\hline My immediate supervisor shows good leadership & Supervision & .991 & $.89 * * *$ & Moved to 'Trust in Supervisor' \\
\hline I respect my immediate supervisor. & Supervision & .970 & $.84^{* * *}$ & Moved to 'Trust in Supervisor' \\
\hline I trust my immediate supervisor & Supervision & .964 & $.86^{* * *}$ & Moved to 'Trust in Supervisor' \\
\hline My immediate supervisor makes clear what is expected of the workplace. & Supervision & .878 & $.76^{* * *}$ & Kept \\
\hline My immediate supervisor ensures our workplace works well together. & Supervision & .878 & $.74 * * *$ & Kept \\
\hline My immediate supervisor keeps me informed about relevant matters. & Supervision & .827 & $.76^{* * *}$ & Kept \\
\hline My immediate supervisor makes sure I understand his/her role. & Supervision & .817 & & Removed \\
\hline My immediate supervisor makes clear what tasks I need to perform. & Supervision & .779 & $.63 * * *$ & Kept \\
\hline My immediate supervisor makes clear what standards are expected in my work. & Supervision & .740 & & Removed \\
\hline My immediate supervisor provides me with regular feedback on my work. & Supervision & .646 & $.68^{* * *}$ & Kept \\
\hline My immediate supervisor actively supports a harassment free work environment & Supervision & .613 & $.48^{* * *}$ & Kept \\
\hline
\end{tabular}

\footnotetext{
${ }^{2}$ Loading reported are from final model after changes listed in the 'implication' column have been applied. Therefore, no loadings are listed for removed items
} 


\begin{tabular}{l|cc} 
Factor loading & $\lambda$ & Implication \\
.714 & $.63^{* * *}$ & Kept \\
.641 & $.60^{* * *}$ & Kept \\
.632 & $.43^{* * *}$ & Kept \\
.575 & $.44^{* * *}$ & Kept \\
.565 & $.47^{* * *}$ & Kept \\
.534 & $.52^{* * *}$ & Kept \\
-.486 & $-.39^{* * *}$ & Kept \\
.476 & $.57 * * *$ & Kept
\end{tabular}

Organisational Commitment

Organisational Commitment

Organisational Commitment

Organisational Commitment

Organisational Commitment

Organisational Commitment

Organisational Commitment

Organisational Commitment
The NZDF is the best possible organisation to work for.
I am willing to put in effort beyond that normally expected in order to help the NZDF be

Being in the NZDF gives me a sense of belonging to one big family.

Deciding to join the NZDF was a definite mistake on my part.

I speak highly of my Service/the NZDF to my friends.

I care about the future of the NZDF.

think I am doing something worthwhile for my country by being in the NZDF. 


\section{Factor 3: Aligned-Cohesive Culture}

OAtS Section

Senior leaders' behaviour reflects the values of the NZDF.

Senior leaders keep people informed about matters that affect their work.

I believe senior leaders have the skills to do the job of leading the NZDF.

Senior leaders are committed to improving the way the NZDF is run.

I have confidence in the leadership of the NZDF.

We celebrate success in the NZDF.

The NZDF cares about the well-being of its people.

People share their knowledge and ideas freely in the NZDF.

I feel I am working for a successful organisation.

I feel informed about the NZDF and its activities.

I can rely on the support of others in the NZDF.

My loyalty to the NZDF is matched by its loyalty to me.
Senior Leaders

Senior Leaders

Senior Leaders

Senior Leaders

Organisational Culture

Organisational Culture

Organisational Culture

Organisational Culture

Organisational Culture

Organisational Culture

Organisational Culture

Organisational Commitment

Factor loading

.972

.959

.949

923

.802

.673

.622

.567

.550

.519

.478

422
Implication

Kept

Kept

Removed

Kept

Kept

Kept

Kept

Kept

Kept

Kept

Kept

Kept 


\begin{tabular}{|c|c|c|c|c|}
\hline Factor 4: WIM & OAtS Section & Factor loading & $\lambda$ & Implication \\
\hline I have experienced harassment in the workplace over the past year. & Equity & -.926 & $.92^{* * *}$ & Covaried \\
\hline I have observed harassment in the workplace over the past year. & Equity & -.925 & $.79 * * *$ & Covaried \\
\hline I have observed bullying in the workplace over the past year. & Equity & -.888 & $.70^{* * *}$ & Covaried \\
\hline I have observed discrimination in the workplace over the past year. & Equity & -.880 & $.76^{* * *}$ & Covaried \\
\hline I have experienced bullying in the workplace over the past year. & Equity & -.875 & $.67^{* * *}$ & Covaried \\
\hline I have experienced discrimination in the workplace over the past year. & Equity & -.855 & $.76^{* * *}$ & Covaried \\
\hline Factor 5: Job Satisfaction & OAtS Section & Factor loading & $\lambda$ & Implication \\
\hline I like the work in my present position. & Job satisfaction & .932 & $.72^{* * *}$ & Kept \\
\hline Most of the time my job is repetitive and boring. & Job satisfaction & -.871 & $-.61 * * *$ & Kept \\
\hline Much of what I do in the NZDF is interesting. & Job satisfaction & 826 & $.55^{* * *}$ & Kept \\
\hline The work I do makes full use of my knowledge and skills. & Job satisfaction & .808 & $.66^{* * *}$ & Kept \\
\hline My job gives me a sense of personal achievement. & Job satisfaction & .795 & $.67^{* * *}$ & Kept \\
\hline Overall, I'm satisfied with my job. & Job satisfaction & .583 & $.63^{* * *}$ & Kept \\
\hline
\end{tabular}




\begin{tabular}{|c|c|c|c|c|}
\hline Factor 6: Leaving Intentions & OAtS Section & Factor loading & $\lambda$ & Implication \\
\hline I will probably leave the NZDF in the next 12 months. & Career Intentions & .865 & $.91 * * *$ & Kept \\
\hline I am actively looking at leaving the NZDF. & Career Intentions & .847 & $.86^{* * *}$ & Kept \\
\hline Within the past year I have considered leaving the NZDF. & Career Intentions & .765 & $.71^{* * *}$ & Kept \\
\hline It would take very little change in my present circumstances to cause me to leave the NZDF. & Career Intentions & .740 & & Removed \\
\hline Factor 7: Health and Safety & OAtS Section & Factor loading & $\lambda$ & Implication \\
\hline I know how to raise any health and safety concerns I may have. & Health and Safety & .912 & $.61^{* * *}$ & Kept \\
\hline I know how to report an accident. & Health and Safety & .868 & $.54 * * *$ & Kept \\
\hline There are personnel within my workplace who have health and safety responsibilities. & Health and Safety & .802 & $.47 * * *$ & Kept \\
\hline Health and safety is treated as an important issue in my workplace. & Health and Safety & .644 & $.44 * * *$ & Kept \\
\hline The hazards of my job are controlled so that I am unlikely to be harmed. & Health and Safety & 611 & $.35 * * *$ & Kept \\
\hline I know what support services are available to me if I were being harassed at work. & Equity & .397 & $.35 * * *$ & Kept \\
\hline
\end{tabular}




\begin{tabular}{|c|c|c|c|c|}
\hline Factor 8: Stress & OAtS Section & Factor loading & $\lambda$ & Implication \\
\hline The level of stress I am experiencing is affecting my personal well-being. & Health and Safety & .850 & $.89^{* * *}$ & Kept \\
\hline The level of stress I am experiencing is affecting my job performance. & Health and Safety & .820 & $.72^{* * *}$ & Kept \\
\hline There is too much stress in NZDF related areas of my life. & Health and Safety & .668 & $.73^{* * *}$ & Kept \\
\hline Factor 9: Work/Life Balance & OAtS Section & Factor loading & $\lambda$ & Implication \\
\hline My work schedule often conflicts with my personal life. & Work/Life Balance & -.877 & - & - \\
\hline I am able to maintain a balance between my personal and working life. & Work/Life Balance & .849 & - & - \\
\hline There is not enough time allocated for the pursuit of fitness activities within the NZDF. & Work/Life Balance & -.673 & - & - \\
\hline My current working hours are too long. & Job Satisfaction & -.648 & - & - \\
\hline The NZDF provides adequate family support. & Work/Life Balance & .601 & - & - \\
\hline The NZDF provides a good quality of life compared with other organisations. & Work/Life Balance & .572 & - & - \\
\hline
\end{tabular}




\section{OAtS Section \\ OAtS Section}

We do not focus enough on equity and diversity issues in our workplace.

Overall, policies are sensitive to the needs of different groups within the NZDF.

I am well informed about equity and diversity policies in the NZDF.

The NZDF is an equal opportunity employer.

\section{Factor 11: Attitude to/about Change}

The rate of change in the NZDF is too fast.

Changes within the last 12 months are likely to produce an NZDF that is more effective as an operational force.

There doesn't seem to be any long term planning in the NZDF.

I am satisfied that changes in the NZDF occur for a reason.

Changes within the NZDF during the last 12 months have had a negative impact on my attitude towards future service.

\section{Factor 12: Pay Faitness}

I am paid appropriately for the job I perform.

The pay and benefits I receive are fair for the work I do.

My pay is comparable to what I would get outside the organisation.
Equity

Equity

Equity

Equity

OAtS Section

Change

Change

Change

Change

Change

OAtS Section

Job Satisfaction

Job Satisfaction

Job Satisfaction
Factor loading

$-.810$

$$
.792
$$

.570

Factor loading

$-.599$

.547

$-.533$

.528

$-.527$

Factor loading

.987

.942

.940
Implication

Kept

Kept

Kept

Kept

i.

Implication 


\begin{tabular}{|c|c|c|c|c|}
\hline Factor 13: Personal Development Opportunities & OAtS Section & Factor loading & $\lambda$ & Implication \\
\hline There are opportunities for advancement / promotion for me within the NZDF. & Career development & 1.113 & - & - \\
\hline There are career and personal development opportunities for me in the NZDF. & Career development & 1.057 & - & - \\
\hline I see change in the NZDF as providing new opportunities for me. & Change & .525 & - & - \\
\hline I have the opportunity to realise my potential in the NZDF. & Job satisfaction & .468 & - & - \\
\hline Factor 14: Work Quality/Standards & OAtS Section & Factor loading & $\lambda$ & Implication \\
\hline The quality of the work done in my workplace is very important to me. & Job satisfaction & .689 & - & - \\
\hline I do my current job well. & Job satisfaction & 663 & - & - \\
\hline I have a great deal of pride in my work. & Job satisfaction & .605 & - & - \\
\hline Factor 15: Competence and Training & OAtS Section & Factor loading & $\lambda$ & Implication \\
\hline This organisation ensures that I am adequately trained for the work I do. & Training and Resources & 686 & - & - \\
\hline People in the NZDF have the necessary skills to meet the NZDF's commitments. & Training and Resources & .660 & - & - \\
\hline $\begin{array}{l}\text { Other members in my workplace have the job relevant knowledge and skills necessary to } \\
\text { accomplish unit targets. }\end{array}$ & Performance Management & .593 & - & - \\
\hline Training opportunities are allocated fairly. & Training and Resources & .570 & - & - \\
\hline
\end{tabular}




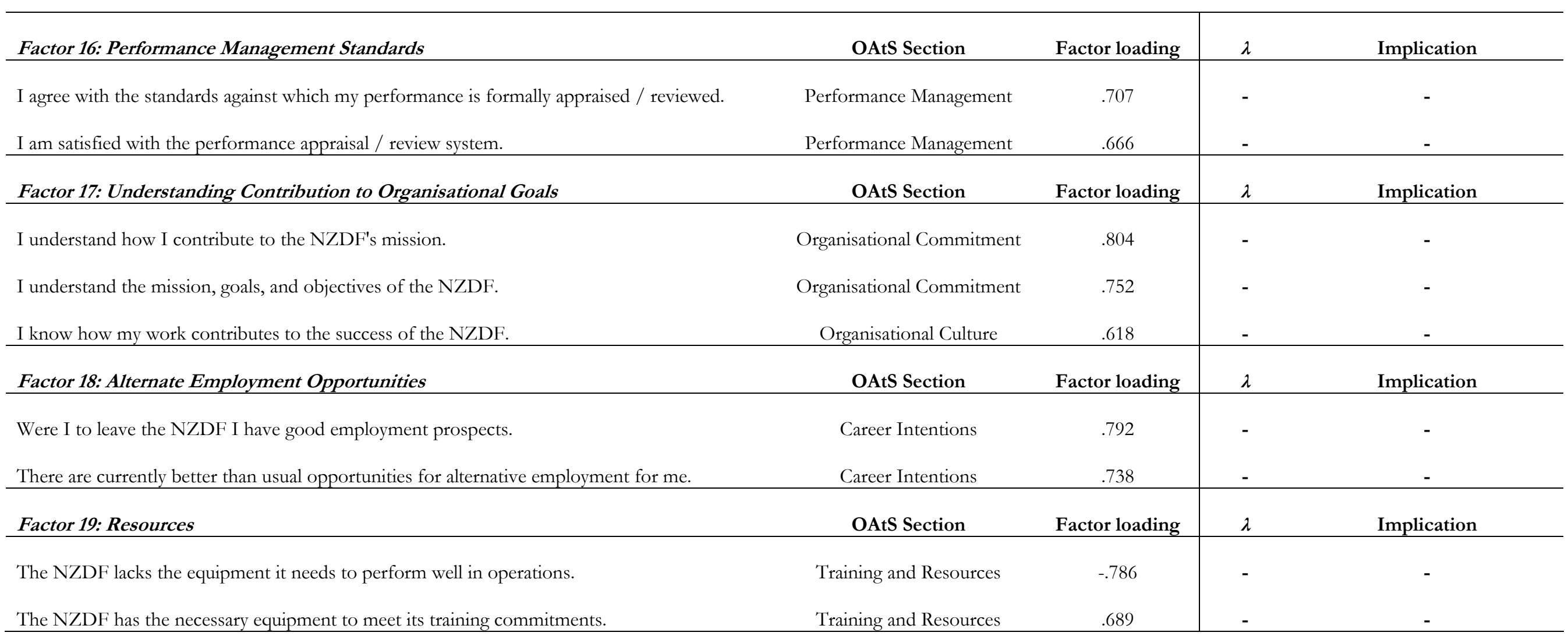


Table 6: Construct fit statistics from PCA and CFA

\begin{tabular}{|c|c|c|c|c|c|c|c|c|}
\hline & \multirow{2}{*}{$\begin{array}{l}\text { PCA Sample } 1 \\
\text { Cronbach's } \alpha\end{array}$} & \multicolumn{7}{|c|}{ CFA Sample 2} \\
\hline & & Cronbach's $\alpha$ & df & $x^{2}$ & RMSEA & TLI & CFI & SRMR \\
\hline Affective Organisational Commitment & .862 & .845 & 20 & $437.400^{* * *}$ & .1 & .897 & .927 & .0444 \\
\hline Job Satisfaction & .861 & .846 & 9 & $207.325^{* * *}$ & .103 & .931 & .960 & .0361 \\
\hline Health and Safety & .796 & .800 & $26^{3}$ & $424.215^{* * *}$ & .086 & .925 & .946 & .0589 \\
\hline Stress & .869 & .869 & & & & & & \\
\hline initial & .923 & & 9 & $1359.138^{* * *}$ & .268 & .736 & .842 & .0660 \\
\hline final & & .905 & 3 & 2.232 & 0 & 1 & 1 & .0032 \\
\hline final ${ }^{4}$ & & $\begin{array}{l}.877^{5} \\
.944^{6}\end{array}$ & 26 & $536.414 * * *$ & .097 & .950 & .964 & .0356 \\
\hline
\end{tabular}

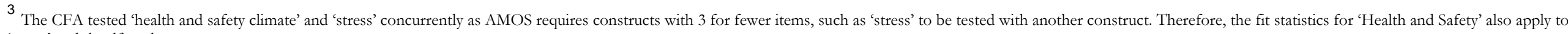
'stress' and the df are large.

${ }^{4}$ Includes 'directive leadership' and 'trust in supervisor'

${ }^{5}$ Cronbach's $\alpha$ for 'directive leadership’
} 


\begin{tabular}{|c|c|c|c|c|c|c|c|c|}
\hline & \multirow{2}{*}{$\begin{array}{l}\text { PCA Sample } 1 \\
\text { Cronbach's } \alpha\end{array}$} & \multicolumn{7}{|c|}{ CFA Sample 2} \\
\hline & & Cronbach's $\alpha$ & df & $x^{2}$ & RMSEA & TLI & CFI & SRMR \\
\hline Aligned-Cohesive Culture & .899 & & 54 & $1221.335^{* * *}$ & .102 & .854 & .880 & .0555 \\
\hline final & & .872 & 44 & $664.507 * * *$ & .082 & .903 & .922 & .0441 \\
\hline Work/Life Balance & .729 & & - & - & - & - & - & - \\
\hline Leaving Intentions initial & .865 & & 2 & $76.282^{* * *}$ & .134 & .949 & .983 & .0287 \\
\hline final $^{7}$ & & .858 & 8 & $48.383^{* * *}$ & .049 & .989 & .994 & .0282 \\
\hline Attitude to/about Change & .719 & & - & - & - & - & - & - \\
\hline Pay Fairness & .878 & & - & - & - & - & - & - \\
\hline Personal Development Opportunities & .785 & & - & - & - & - & - & - \\
\hline Work Quality/Standards & .709 & & - & - & - & - & - & - \\
\hline Competence and Training & .704 & & - & - & - & - & - & - \\
\hline Performance Management Standards & .813 & & - & - & - & - & - & - \\
\hline
\end{tabular}

${ }^{6}$ Cronbach's $\alpha$ for 'trust in supervisor'

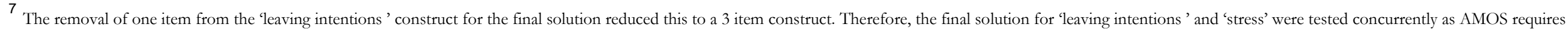
constructs with 3 for fewer items to be tested with another construct. 


\begin{tabular}{|c|c|c|c|c|c|c|c|c|}
\hline & \multirow{2}{*}{$\begin{array}{l}\text { PCA Sample } 1 \\
\text { Cronbach's } \alpha\end{array}$} & \multicolumn{7}{|c|}{ CFA Sample 2} \\
\hline & & Cronbach's $\alpha$ & df & $x^{2}$ & RMSEA & TLI & CFI & SRMR \\
\hline $\begin{array}{l}\text { Understanding Contribution to } \\
\text { Organisational Goals }\end{array}$ & .775 & & - & - & - & - & - & - \\
\hline Alternate Employment Opportunities & .682 & & - & - & - & - & - & - \\
\hline Resources & .559 & & - & - & - & - & - & - \\
\hline
\end{tabular}

Note: ${ }^{* * *} p<0.001$ 


\section{Discussion}

Nineteen factors were identified by the PCA, and of these 8 were hypothesised as antecedents or consequences of WIM. The inclusion of WIM itself gives 9 factors of particular interest for this thesis. These 9 factors are:

1. WIM,

2. Aligned-cohesive culture,

3. Directive leadership,

4. Health and safety climate,

5. Equity and diversity climate,

6. Job satisfaction,

7. Affective organisational commitment,

8. Stress, and

9. Leaving intentions.

These factors were further analysed using CFA. Five of the factors showed acceptable fit and four required further improvement. The four constructs were WIM, directive leadership, aligned-cohesive culture and leaving intentions. Each of the four factors was altered based on the conceptual basis for the construct, informed by statistical output from the CFA such as modification indices and residuals. The changes that were made resulted in measures which show acceptable fit. A discussion of the improvements for each of the four constructs follows.

As the factors are created from items in a survey which was developed without specific reference to the literature in the domains that it taps, it is important to establish the equivalence between these measures and those used in the literature. This is done by comparing the content of these measures with those commonly used in the literature. Therefore, the measurement literature on WIM and the other relevant constructs is examined. 
The following discussion considers the content validity of each of the constructs, and for the four constructs which required improvement, justification for alterations are provided.

\section{WIM}

Results of the PCA suggest that all items loaded strongly onto one factor. However further investigation using CFA in the subsequent samples indicated that the construct was more complex.

\section{Improvement in Fit}

The initial solution for a single WIM construct in the CFA showed poor fit (see table 6). Inspection of the statistical information for model improvement was considered with reference to conceptual information about the construct. Two sources of conceptual information were available within the survey which may inform the improvement in fit: inspection of the items themselves and inspection of the definitions provided with the items.

\section{$\underline{\text { WIM Items }}$}

The WIM items covered two types of distinctions. The first was between the type of WIM occurring: harassment, discrimination or bullying. The second was between the types of exposure: either self-experienced or observed occurring to others. These distinctions gave the 6 items in the matrix below.

Table 7: Components of the WIM measure

\begin{tabular}{lll}
\hline Harassment & Observed & Experienced \\
& I have observed harassment in & I have experienced \\
the workplace in the last year. & $\begin{array}{l}\text { harassment in the workplace } \\
\text { in the last year. }\end{array}$ \\
Discrimination & I have observed & I have experienced \\
discrimination in the & discrimination in the \\
wullying & I have observed bullying in & workplace in the last year. \\
& the workplace in the last year. & the workplace in the last year.
\end{tabular}


Inspection of the standardised residual covariances and modification indices indicated that the single factor WIM was unable to account for much of the covariation that existed between pairs of items. Modification of the model to reflect the distinction between observed and experienced WIM, or the three types of WIM did not improve the fit of the overall construct to acceptable levels. ${ }^{8}$ Figure 7 displays the pattern of covariation suggested by the standardised residuals and modification indices. The figure indicates that the pattern of item covariances did not conform to the distinction of observation vs experience or to the distinction of the three types of WIM as one might expect. In the figure solid arrows represent covariation which is not explained by the single-construct solution (as indicated by standardized residuals and modification indices). The dashed arrow represents unexplained co-variation which completes the pattern of unexplained variance but which was not suggested by the standardised residuals and modification indices. It is depicted in the figure to demonstrate the pattern between the items.

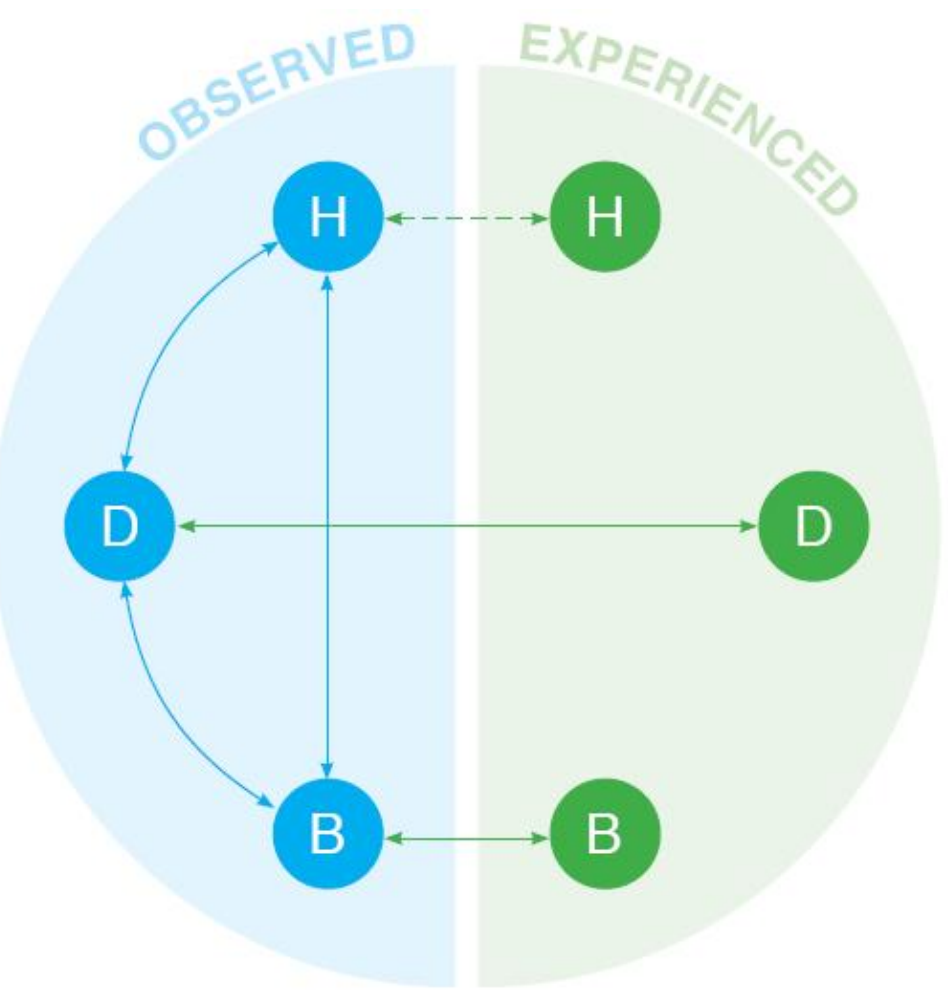

Figure 7: Unexplained variance in the WIM measure

\footnotetext{
${ }^{8}$ Model reflecting observed/experienced WIM distinction: $\chi^{2}(4)=112.173, p=.000$, RMSEA $=.278$, $\mathrm{TLI}=.717, \mathrm{CFI}=.849$, and SRMR $=.0649$. Model reflecting type of WIM distinction (harassment/discrimination/bullying): $\chi^{2}(6)=629.518, p=.000$, RMSEA $=.223$, TLI $=.817$, CFI $=$ .927 , and SRMR $=.0379$.
} 
Given that the content of the items cannot account for the covariation between the items, an alternative explanation was required.

\section{$\underline{\text { WIM Definitions }}$}

Another possible source of variation was the content of the definitions provided to participants in the survey. The OAtS survey provides definitions for harassment, discrimination and bullying to participants. This approach of defining the concepts for participants is used in other measures (e.g. Einarsen, Raknes, \& Matthiesen, 1994; Salin, 2003) and derived from bulling research in school settings (Einarsen, Hoel, Zapf \& Cooper, 2003).

The content of the definitions was inspected for any evidence that may exist to explain the covariation between items as detailed in figure 7. Examination of these definitions revealed that the content of harassment and bullying definitions are quite similar to one another and that these are quite different from discrimination? The pattern of similarities and differences across these definitions cannot provide clues as to the reason for the covariances seen in the figure 7 .

Therefore, neither the distinction among the items nor the item definitions can explain the pattern of covariation among the items. In order to covary the items, conceptual justification must be provided. Joreskog (1993) describes the high

\footnotetext{
9

${ }^{9}$ The following definitions were provided to participants in the OAtS survey. The areas of overlap in the harassment and bullying definitions are highlighted below by the use of different fonts. The areas of overlap include reference to issues of humiliation (bold), intimidation (italics) repetition (underlined) and harm (CAPITALISED).
}

Harassment is behaviour that is demeaning to another person's status, leaving the person feeling humiliated, offended or intimidated. Harassment can be repetitive or of a significant nature, is often power based, and is DETRIMENTAL TO THE INDIVIDUAL.

Workplace bullying is usually repeated (or a significant single incident), unreasonable behaviour (victimising, humiliating, undermining or threatening) directed toward a person that creates a risk to the MENTAL OF PHYSICAL HEALTH AND SAFETY OF THE PERSON

Discrimination occurs when a person is treated less favourably than others in the same or similar circumstances based on the specific grounds under the Human Rights Act. The discrimination definition does not refer to any of these issues. However, the co-variation displayed in figure 7 indicates that observed discrimination co-occurs with the other types of WIM.

Although this finding is not helpful for explaining the covariation it is interesting as it suggests that participants are not necessarily using the definitions when they respond to the items about the different types of WIM. If they were one would expect a strong correlation between experienced harassment and experience bullying as any behaviour that meets the criteria for one, would almost certainly meet the criteria for the other, given the similarities between the definitions. Although there is a correlation between these two items $(\mathrm{r}=.578)$, in the context of the correlations between the other items in this group this correlation is not especially strong; as $53 \%$ of the remaining correlations are stronger than .578 . 
standard of justification required for the covariation of error terms in cross-sectional studies [emphasis added]:

the error terms should be uncorrelated from one indicator to another. This is part of the definition of being indicators of a construct. If the errors terms for two or more indicators correlate, this means that these indicators measure something else or something in addition to the construct they are supposed to measure. If this is the case, the meaning of the construct and its dimensions may be different from what is intended.... Every correlation between error terms must be justified and interpreted substantively.' (p297)

As the items all loaded onto a single construct in the EFA it is likely that the indicators are measuring something in addition to the WIM construct. Further review of the literature around WIM provided support for the complex pattern of relationships among the WIM items.

\section{Literature Relevant to the Structure of WIM}

Although some existing work has been done in the area of observation and experience of WIM, research has tended to focus on contrasting the experiences of observers and targets. Research has demonstrated that observers of WIM suffer negative consequences and that the effects of observing and experiencing WIM are cumulative (Einarsen, Raknes and Mattheisen, 1994; Cooper-Thomas, Catley, Bentley, Gardner, O'Driscoll \& Trenberth, 2011). The finding that some participants in these studies report both experiencing and observing WIM provides evidence that they can co-occur. The finding that experience and observation did not always cooccur suggests that the relationship is more complex. This thesis investigated WIM from a different perspective from existing work as it looked at the structure of WIM as a construct tapping both observed and experienced WIM. Therefore this thesis extends and contributes to the understanding of the complexity of the relationship between observed and experienced WIM.

In this thesis the co-occurrence of the observation of different types of WIM supports the suggestion that observing WIM is one construct (blue section of figure 
$7)^{10}$. In an environment where WIM occurs three types of WIM are visible; harassment, discrimination and bullying. Ensher, Grant-Vallone and Donaldson (2001) argue that the mechanism by which observing WIM is associated with negative outcomes is the work environment: WIM is theorised to create a negative work environment which is experienced by all employees not just those who personally experience WIM. Therefore observation of WIM can reflect a dysfunctional work environment. The finding that negative workplace environments are associated with more bullying has been demonstrated even when those who are bullied are excluded from the analysis as their view of workplace environment may be clouded by their experience of bullying (Mageroy, Lau, Riise and Moen, 2009). Therefore observed WIM may exist as an indicator of a negative workplace environment.

In contrast to the co-occurrence of observed WIM in this thesis, when participants are asked about what they have experienced there is a distinction between the types of WIM (green section of figure 7). There is no covarying of error terms across any of the types of WIM. This result suggests that specific types of WIM behaviours may be experienced in isolation from one another. This finding fits well with organizational theories and empirical findings that suggest that perpetrators choose the WIM behaviours to use in a given interaction based on features of the targets (Berdahl \& Moore, 2006). For example, gender or ethnic discrimination may be used by Caucasian males to target females or males from ethnic minorities but bullying may be used to target people who share demographic characteristics with the perpetrator. Therefore, reported experienced WIM is limited by the demographic of the respondent: the respondent is more likely to experience certain types of WIM based on their membership of a demographic group. In contrast, observing WIM is not limited by the demographic of the respondent: the respondent can observe WIM occurring to members of demographic groups that differ from their own. The restriction of membership to one demographic, but observation of multiple demographics suggests that individuals will report observing multiple types of WIM,

\footnotetext{
10 An alternate explanation is that this result may be an artefact of the correlational bias in observer ratings (Berman \& Kenny, 1976). Personality research has noted that observer ratings of conceptually related constructs are often more highly intercorrelated than similar self-ratings. However, conclusive explanations for why this phenomenon might occur have not been offered.
} 
but experience only isolated types of WIM. This is the pattern observed in the data demonstrated in figure 7.

Considering the relationships between observation and experience, a link existed between observation and experience for two types of WIM: discrimination and bullying (links across blue/green sections of figure 7). This can be explained by the sensitivity argument. That is, people who have experienced something are sensitised to it and are more likely to see it around them (Raver \& Nishii, 2010). Additionally, the concept of social ostracism (Williams, 1997) can inform another explanation for the co-occurrence of experienced and observed WIM. Experiencing WIM causes people to feel socially ostracised (Einarsen \& Mikkelsen, 2003). Therefore, interpreting (perhaps neutral) behaviour, which occurs to another, as WIM can offer benefits to the socially ostracised observer. Believing that another person has also experienced WIM reduces feelings of social ostracism and associated stress. This reduction occurs because the first person no longer feels alone in their experience of WIM. As such, people who experience WIM may be motivated to perceive it around them in order to reduce their own stress levels. However, an explanation is needed for why this is not happening for harassment, especially when considering the similarity in definitions that exists between harassment and bullying.

An understanding of harassment itself can provide part of the explanation. Lapierre, Spector and Leck (2005) make the argument that the distinction between sexual and non-sexual harassment may be due to amount of attention each gets from the law, the media and in turn organisations. Sexual harassment gets the most attention in all three areas. If the term 'harassment' primes respondents to think of sexual harassment, precisely because sexual harassment gets most coverage in the media, law and organisational anti-harassment training, personnel may be responding to the harassment item as though it refers to sexual harassment.

Lapierre, Spector and Leck (2005) also note that as sexual harassment is less common than non-sexual harassment it is more likely to be attributed to a specific perpetrator. The more common non-sexual WIM behaviours are seen as a systemic organisational issue precisely because they occur more commonly and cannot therefore be blamed on one individual. If person A experienced sexual harassment themselves and they attribute it to a single perpetrator (person B) rather than the 
organisation, person B would also have to be observed by person A harassing someone else (person C) in order for person A to report observed harassment. This scenario is less likely to happen as 1) sexual harassment is uncommon in relation to other types of WIM; and 2) another person of the same demographic (e.g. female) would need to be in present in the workplace for the perpetrator to sexually harass. If the perpetrator intended to intimidate a male, sexual harassment would not be the WIM behaviour of choice. Another consideration is the visibility of sexual harassment in contrast to other forms of WIM. Sexual harassment may be more likely to occur behind closed doors and therefore respondents do not have the opportunity to observe and then report such observed behaviour.

Therefore, due to the uncommon nature of sexual harassment, the fact that perpetrators choose their methods based on the features of their targets, and the invisible nature of sexual harassment, the co-occurrence of reported experienced and observed WIM is unlikely to happen. The result of these points about the frequency of sexual harassment means that the sensitivity argument for the link between experienced and observed WIM may not apply in the case of harassment.

However, for reasons of parsimony, WIM was remodelled to include covarying of error terms across observation and experience for all 3 types of WIM including harassment. Model fit was suitably improved for this final solution as detailed in table 6.

Another issue related to the content validity of the WIM measure is the use of a perceptual measure of WIM.

\section{$\underline{\text { Subjective and Objective Approaches to WIM Measurement }}$}

Two main methods of measurement are used in the WIM literature; perceived measures and behaviour based measures of WIM. Perceived measures, sometimes referred to as direct query measures, ask directly about experiences and use labels such as harassment or bullying in the item. Behavioural measures do not refer to labels but instead ask respondents whether they have experienced various behaviours which meet the technical definition of some type of WIM. The measure used in this thesis current research is a perceptual or direct query measure. 
A series of meta-analyses (Bowling and Beehr, 2007, Lapierre, Spector \& Leck, 2005) identified the most common WIM measures in the literature:

1. Interpersonal Conflicts at Work Scale (ICAWS) which focuses on getting into arguments with others and how often others act nasty to the respondent (Spector \& Jex, 1998)

2. Negative Acts at Work Questionnaire (NAQ) which focus on work, person related and physically intimidating bullying (Einarsen, Hoel \& Notelaers, 2009)

3. Abusive supervision scale (ASS) which focuses on interpersonal bullying from a supervisor (Tepper, 2000).

4. Workplace Incivility Survey WIS which identifies ambiguously intended workplace harm (Cortina Magley, Williams, \& Langhout, 2001)

All of these measures ask about specific types of behaviours over a given time period (e.g. ICAWS: 'How often do other people yell at you at work?' and 'How often do other people do nasty things to you at work?'. NAQ: 'Spreading gossip or rumours about you'. ASS: 'doesn't give me credit for jobs requiring a lot of effort'. WIS: Ignored or excluded you from professional camaraderie'). In addition to these behavioural items only the NAQ also provides a definition of bullying at work after the list of behavioural items and the respondent must indicate whether or not they consider themselves as victims of bullying at work according to this definition (Einarsen, Hoel \& Notelaers, 2009). Therefore, it appears that in the literature behavioural measures are more common.

However, an important consideration for the appropriateness of the type of measure is the aims of the research. Lengnick-Hall (1995) suggests that there are two possible ways to define the construct of sexual harassment: legally and objectively or perceptually and subjectively. Lengnick-Hall recommends that researchers must identify the aims of their research, and choose the type of WIM definition which best meets the needs of the research questions. Ilies, Hauserman, Schwochau and Stibal (2003) support this view and note that different research may benefit from different approaches to measurement and recommend that behavioural measures are more 
appropriate for investigating legal outcomes whereas perceptual measures are more appropriate for predicting organisational outcomes. This view is echoed by authors that study non-sexual WIM. Einarsen et al (2003) suggests that subjective conceptualisations are most appropriate for attempting to explain the variance in individual and organisational outcomes such as absenteeism and turnover. Given that the aims of this thesis involve identifying the antecedents and consequences of WIM, a perceptual measure is appropriate.

A theoretical case has been made for the use of a perceptual measure of WIM. A related issue is whether perceptual measures of WIM have been empirically linked to individual and organisational antecedents and consequences. Research which has used perceptual measures across a range of types of WIM has demonstrated these relationships empirically. For example, a study of men and women in the US Armed Forces which investigated the effects of labelling an incident as sexual harassment identified that labelling an experience as sexual harassment does not increase the negative outcomes (satisfaction, wellbeing, cohesiveness and organisational commitment) of the experience (Munson, Miner \& Hulin, 2001). Additionally, in the discrimination literature perceived discrimination was related to lower organisational commitment, job satisfaction and organisational citizenship behaviours (Ensher, Grant-Vallone \& Donaldson, 2001). It therefore seems perceptions of WIM are demonstrably related to many work-related variables. Ensher, Grant-Vallone and Donaldson (2001) state that perceptions are 99 percent of reality and Einarsen, Hoel, Zapf and Copper (2003) note that perception is at the heart of the matter of WIM so there is a compelling case for the importance of perception in our understanding of WIM.

Another consideration that impacts on the appropriateness of the measure is the specific behavioural norms in the organisation. Rayner and Hoel (1997) highlighted the relevance of existing social norms in the study of aggression by suggesting that culture can act as a screen through which behaviours are interpreted. In the military context, certain behaviours may not carry the same meaning as in other work contexts. For example, items which appear on behavioural measures such as yelling, swearing and physical violence can have a legitimate place in military training. In military samples, respondents may endorse these items even though these behaviours would not be considered WIM in the specific training context. Therefore, the 
subjective interpretation provided by the respondent who understands the norms of the organisation they work in is crucial.

Although behavioural measures are more common, the perceptual measure used in this thesis is the most appropriate measure to answer the research questions posed, in the sample being used.

\section{$\underline{\text { Summary }}$}

The WIM construct is complex. The items themselves, and the definitions provided in the literature cannot explain the complex nature of the WIM construct. Instead, the findings fit with existing theoretical and empirical work on the underlying nature of WIM behaviours. The WIM construct is therefore modelled as displayed in figure 7. These findings on the nature of WIM across observation and experience and three different sub-types, contribute to the existing understanding of structure of WIM. In addition, the use of a perceptual measure of WIM in this thesis is appropriate for answering the research questions posed, given the sample of interest.

\section{Antecedents}

\section{Aligned-Cohesive Culture}

\section{Improvements in Fit}

Twelve items in the PCA loaded onto a single factor 'aligned-cohesive culture'. The CFA indicated that the initial solution for aligned-cohesive culture showed inadequate fit. Inspection of residuals and modification indices signify that items ' $\mathrm{I}$ believe senior leaders have the skills to do the job of leading the NZDF' and 'Senior leaders are committed to improving the way the NZDF is run' are problematic. These two items appear to be tapping into the same thing as they are the most highly correlated $(r=.677)$ and they have a high residual. The aligned-cohesive culture scale contains a large number of items so two items which tap the same domain are unnecessary and can be removed. The item 'I believe senior leaders have the skills to do the job of leading the NZDF' is most similar to another item: 'I have confidence in the leadership of the NZDF' (these are also quite highly correlated: $r=.607$ ) 
therefore the item 'I believe senior leaders have the skills to do the job of leading the NZDF' is removed. These changes improved fit considerably for the final solution.

\section{$\underline{\text { Content Validity }}$}

The measurement of alignment has been primarily qualitative (e.g. Semler, 1997) or if quantitative, has assessed the degree to which business unit practices are aligned with the core messages of the organisation (Beehr, Glazer, Fischer, Linton \& Hansen, 2009). The measure used in this thesis is a quantitative measure that reflects the key theoretical domains of alignment and cohesion.

The aligned-cohesive culture construct identified in this thesis contains facets of confidence in and loyalty to senior leadership, supportive organisational culture, communication, and performance. The referent in this group of items is senior leadership and the NZDF. The inclusion of both of these referents in this measure suggests that respondents view senior leaders as NZDF; they do not appear to make a distinction between those leading the organisation and the organisation itself. Examination of the items in the aligned-cohesive culture construct indicates that it contains the conceptual elements of Semler's (1997) theoretical aspects of alignment. These include: strategy, reward systems, values and norms, and performance. In addition to these features, the measure also contains communication which is a key component of an aligned-cohesive culture, as communication is the way which alignment can be achieved. Without communication about the organisations goals employees and subunits would be unable to align to them. Communication about organisational goals has been demonstrated empirically to be an antecedent of alignment (Beehr, Glazer, Fischer, Linton \& Hansen, 2009). In the current study alignment is conceptualised more broadly as descriptive of the organisational culture, and therefore communication about organisational goals is considered to be a feature of the overall aligned-cohesive culture rather than an antecedent to it. Items in the current study which assess each of the theoretical aspects are detailed in the table below.

The cohesion aspects of the aligned-cohesive culture refer primarily to vertical bonding, which is perceptions of confidence in, and loyalty to, leaders (Manning, 
1994). Items which tap vertical bonding are shown in bold. Horizontal bonding or cohesion amongst peers occurs as a result of the culture of the organisation (named 'values and norms' in Semler's (1997) model). These items are shown in italics.

Table 8: Aspects of alignment and cohesion as measured by 'aligned-cohesive culture'

Aspect Item

Strategy Senior leaders are committed to improving the way the NZDF is run

I have confidence in the leadership of the NZDF.

Reward systems We celebrate success in the NZDF.

Values and norms Senior leaders' behaviour reflects the values of the NZDF

The NZDF cares about the well-being of its people.

I can rely on the support of others in the NZDF.

My loyalty to the NZDF is matched by its loyalty to me.

Performance I feel I am working for a successful organisation.

Communication People share their knowledge and ideas freely in the NZDF.

Senior leaders keep people informed about matters that affect their work

I feel informed about the NZDF and its activities.

Therefore, the align-cohesive culture measure shows good content validity as it covers the key domains in both alignment and cohesion theory and shows good fit.

\section{Directive Leadership}

\section{Improvements in Fit}

The initial solution for directive leadership behaviour showed poor fit. When inspecting the items included in the construct, 3 stand out as being related to 
subordinates satisfaction with their supervisor rather than subordinates evaluations of their supervisor's behaviour. Satisfaction with the supervisor is likely to occur as a result of leadership behaviour. That is, if the supervisor sets and communicates expectations, promotes teamwork and provides performance feedback to subordinates then this leads to the development of a perception of good leadership. The 3 items which conceptually relate to satisfaction with leadership are:

1. My immediate supervisor shows good leadership,

2. I respect my immediate supervisor, and

3. I trust my immediate supervisor.

As these 3 items measures a different concept, they are removed from 'directive leadership' to create a new construct. The new construct is named 'Trust in supervisor'.

Sample correlations for the remaining items indicate that item redundancy may be a problem. Standardised residuals and modification indices suggest the items 'My immediate supervisor makes clear what tasks I need to perform' and 'My immediate supervisor makes clear what standards are expected in my work' are problematic. Both of these items relate to role ambiguity which has been defined as:

"relative unpredictability of the outcome of an individual's bebaviour .....and
includes components of lack of input from the environment to guide behaviour" (Gilboa, Shirom, Fried \& Cooper, 2008, p.230).

Existing measures of Role ambiguity refer to both priorities and standards, but in these measures the reference made to standards is standards of behaviour rather than standards of work (Giga, Cooper \& Faragher, 2003). The item 'My immediate supervisor makes clear what standards are expected in my work' did not relate well to this key component of the role ambiguity concept that supervisors have an impact on. Therefore it was removed. Additionally, the concept of standards of work would likely be encompassed by the item 'My immediate supervisor provides me with regular feedback on my work.'

When inspecting the remaining items the item 'My immediate supervisor makes sure I understand his/her role' stands out as an item which conceptually does not fit with the others. This behaviour is only superficially related to subordinates as context for 
the teams' work. Therefore this item was removed. These changes improved fit to acceptable levels

\section{Content Validity}

The leadership construct in this thesis makes reference to the subordinates' perceptions of their supervisor's performance. Specifically, the supervisor's responsibilities to set and communicate expectations, promote teamwork and a constructive team environment and provide performance feedback to subordinates. The items in this scale address the lower-order needs of subordinates (directive or transactional leadership) rather than the higher order needs such as providing vision (transformational leadership; Kalliath, Brough, O’Driscoll, Manimala \& Siu, 2010).

Inspection of measures of transactional and directive leadership (Pearce \& Sims, 2002) indicate that many of the items in the current scale show similarity.

Table 9: Directive and transactional leadership items

\begin{tabular}{lcc}
\hline & Current Study & Pearce \& Sims, 2002 \\
\hline Transactional & My immediate supervisor makes & My team leader provides commands in \\
leadership & clear what tasks I need to perform & regard to my work \\
Directive & My immediate supervisor provides & My team leader gives me positive feedback \\
leadership & me with regular feedback on my & when I perform well \\
& work. & \\
\hline
\end{tabular}

Additionally, many items in the construct are similar to items used by researchers investigating leadership behaviour's relationship to WIM. For example, Einarsen, Raknes and Matthiesen (1994) subordinates reports of leadership behaviour to be consistently related to bullying across different employee types. Leadership behaviours were measured with 6-item scales developed by Raknes and Matthiesen (1994):

1. The quality of guidance and support,

2. The clarity of your working instructions,

3. The management's organisation and adaptation of the work you are to perform,

4. The feedback you receive on the work and the tasks you perform, 
5. The recognition you received for your performance at work and

6. The co-operation relationship with your closest superior.

Einarsen, Raknes and Matthiesen's (1994) construct contains elements of communication of expectations and performance feedback but does not cover the team development aspects of leadership as measured by the construct in this thesis.

The construct is very similar to the 'involved leadership' scale used by Winsborough, Morris and Hughes (2008). There were 8 items in common with this scale. The construct is also similar to the 'participative leadership' scale used by Hoel, Glaso, Hetland, Cooper, and Einarsen (2010) in that it focuses on sharing information with employees. Therefore, there is good evidence for the content validity of the leadership construct identified in this thesis both in terms of its content and its relationship to WIM.

\section{Equity and Diversity Climate}

The construct identified in this thesis as equity and diversity climate centres on the focus on and information sharing about equity issues, the sensitivity of policies, and the judgement that NZDF is an EEO employer. In WIM research equity and diversity climate measures have been used most extensively in the sexual harassment and discrimination literature.

A meta-analysis by Willness, Steel and Lee (2007) reports that sexual harassment climate is most commonly measured using the Organisational Tolerance for Sexual Harassment Inventory (OTSHI). The OTSHI has three components: risk to victims for complaining, lack of sanctions against offenders, and perceptions that the complaint will not be taken seriously (Hulin, Fitzgerald \& Drasgow, 1996). These components are more proximal and specific than the general and distal components contained in the construct in this thesis.

In the military setting the Military Equal Opportunities Climate Survey (MEOCS) has been used to measure $\mathrm{EO}$ climate in relation to race and gender discrimination in particular (Truhon, 2008). The MEOCS asks participants to report on expected behaviour. Of particular interest is the Positive Equal Opportunity Behaviour subscale which contains items such as 'A supervisor gave the same punishment to 
minority and majority subordinates for the same offence' and 'Majority and minority members were seen socialising together'. These items reflect behaviours which are similar to the behavioural based measures of WIM itself rather than equity and diversity climate.

The approach to measuring equity and diversity climate in this thesis reflects the approach taken by Law, Dollard, Tuckey and Dormann (2011) to measuring Psychological Safety Climate (discussed in detail in the forthcoming health and safety climate section). The equity and diversity climate construct taps an equivalent measure of three of the four domains of Psychological Safety Climate. The domains are management priority, organisational communication and management commitment. The focus of these domains indicates the value that the organisation places on Equity and Diversity as well as the communication of this value throughout the organisation. The inclusion of both the value and communication facets make the equity and diversity climate construct a good measure of climate for use in research which focuses on the impact of climate on behaviour as the value and communication components capture the function of social norms, which are to communicate organisational values to personnel.

\section{Health and Safety Climate}

The health and safety climate construct identified in this thesis covers 3 of the subscales of Law, Dollard, Tuckey and Dormann's (2011) psychological safety climate. These subscales and example items from Law, Dollard, Tuckey and Dormann's (2011) study and the current study are displayed in table 10. Although, the management commitment domain is not explicitly covered in the current study, management commitment is implied through management priority, organisational communication and organisational participation. 
Table 10: Health and safety climate items compared to Psychological Safety construct

Example items

\begin{tabular}{|c|c|c|}
\hline Domain & $\begin{array}{l}\text { Law, Dollard, Tuckey and } \\
\text { Dormann (2011) }\end{array}$ & Current Research \\
\hline Management priority & $\begin{array}{l}\text { 'Senior management considers } \\
\text { employee psychological health } \\
\text { to be as important as } \\
\text { productivity' }\end{array}$ & $\begin{array}{c}\text { 'Health and safety is treated as } \\
\text { an important issue in my } \\
\text { workplace' }\end{array}$ \\
\hline Organisational communication & $\begin{array}{l}\text { 'There is good communication } \\
\text { here about psychological safety } \\
\text { issues which affect me' }\end{array}$ & $\begin{array}{l}\text { 'I know how to raise any health } \\
\text { and safety concerns I may have' }\end{array}$ \\
\hline Organisational participation & $\begin{array}{l}\text { 'Employees are encouraged to } \\
\text { become involved in } \\
\text { psychological safety matters' }\end{array}$ & $\begin{array}{l}\text { 'There are personnel within my } \\
\text { workplace who have health and } \\
\text { safety responsibilities' }\end{array}$ \\
\hline Management commitment & $\begin{array}{l}\text { 'Senior management acts } \\
\text { decisively when a concern of an } \\
\text { employee's psychological health } \\
\text { is raised' }\end{array}$ & - \\
\hline
\end{tabular}

One key difference is that the measure in this thesis is broader as it is not limited to only psychological health but also includes physical health. All items in the health and safety climate scale used in this thesis could refer to either psychological or physical health. It is likely that due to the focus on stress and mental health (through the provision of mental health support in the organisation - an unusual occurrence specific to the military) that participants are more likely to view health and safety as referring to both physical and psychological health than in personnel in other nonmilitary organisations.

WIM has recently been recognised as an example of a source of harm under health and safety legislation. In 2003 New Zealand's Health and Safety in Employment Amendment Act 2002 was amended to extend the definition of 'harm' to include mental or physical harm caused by work-related stress. The Act's definition of hazard was also extended to include 'a situation where a person's behaviour may be an actual or potential cause or source of harm to the person or another person' (Department 
of Labour, 2003 pp 29). Therefore, a person inflicting WIM on others can be considered a hazard under the Act. The change to this legislation signals that organisations have an obligation to value workplace health and safety in relation to WIM related harm.

\section{Consequences}

\section{Stress}

This construct has 3 items which reflect the effect of stress on both personal wellbeing and job performance, as well as an overall assessment of the appropriateness of the level work related stress. The measure reflects the strain component of the organisational stress process, as it focuses on the outcomes of experiencing stress (Kalliath, Brough, O’Driscoll, Manimala \& Siu, 2010).

Three general-stress measures that are well used in work settings and therefore make good comparisons with the scale used in this thesis are the Perceived Stress Scale (Cohen, Kamarck, \& Mermelstein, 1983), the Job Stress scale (Parker \& DeCotiis, 1983) and the Stress in General Scale (Stanton, Balzer, Smith, Parra \& Ironson, 2001; Lim \& Cortina, 2005).

The Stress in General Scale asks participants to respond to a list of stress related adjectives (e.g. 'tense', 'calm' or 'hectic') as to the degree to which they describe their 'job in general' (Stanton, Balzer, Smith, Parra \& Ironson, 2001). The Job Stress scale contains two dimensions: anxiety and time (Parker \& DeCotiis, 1983). Example items include 'My job gets to me more than it should' for anxiety and 'I have too much work and too little time to do it' for time. The Perceived Stress Scale contains a series of stress related items which respondents report experiencing over the past month, example items include feeling 'nervous or "stressed"?' and 'difficulties were piling up so high you could not overcome them?' (Cohen, Kamarck, \& Mermelstein, 1983).

The common measures used in the literature are all more comprehensive measures of stress than the stress measure identified in this thesis. However, they all rely on individuals' perceptions of stress, and are transparent to respondents in terms of what the measure is assessing. Although these measures differ to the one used in this thesis, there are compelling reasons why the current measure is appropriate for 
organisational research. First, stress is an area where respondents have a good understanding of the content of the construct (Kalliath, Brough, O’Driscoll, Manimala \& Siu, 2010). Second, the short measure covers key domains of theoretical models of stress. Third, the measure concentrates on areas which are particularly important for organisationally focused stress research.

The general public is becoming increasingly well informed about stress and Kalliath, Brough, O'Driscoll, Manimala and Siu (2010) argue that general questions such as 'how do you know when you're stressed?' and 'how do you manage stress?' are able to tap key areas of theoretical stress models. These authors assert that an individual is the best person to identify their own stress. Therefore, stress may be an area of organisational research where short direct measures are particularly appropriate.

The three items in the stress scale cover 3 important domains of Cooper and Mashall's (1976) work-stress model. These are the organisational sources of occupational stress, and both the organisational and individual strain outcomes of experienced stress. Coverage of these important domains in such a short measure provides a useful tool for research where stress is a component of a model rather than the focus of the research.

The stress scale used in this thesis focuses on the stressors the organisation can control (occupational stressors) and both the work-related and individual strain outcomes. This provides a good measure of the influence of work-related stress on respondents. This measure will be particularly useful for research which focuses on organisational antecedents which have a bearing on stress, such as this thesis.

\section{Job Satisfaction}

The job satisfaction construct identified in this thesis contains both a global measure of job satisfaction 'Overall, I'm satisfied with my job' as well as some facets particularly intrinsic satisfaction, for example 'Much of what I do in the NZDF is interesting' and 'My job gives me a sense of personal achievement'. This focus is not surprising given that it is a military sample, and much of the motivation for military personnel is about service. The construct shares three items with the Winsborough, 
Morris and Hughes (2008) job satisfaction measure: 'Most of the time my job is repetitive and boring', 'much of what I do in the NZDF is interesting' and 'I like the work in my present position'. Therefore, the job satisfaction construct is similar to previously validated measures of job satisfaction.

\section{Affective Organisational Commitment}

The construct of organisational commitment has three components: affective commitment, continuance commitment and normative commitment (Allen \& Meyer, 1990). Affective commitment is the extent to which members are emotionally attached to the organisation and identify with it - their commitment is driven by personal meaning and sense of belonging.

The items in this construct conform well to the definition of affective organisational commitment. However, there are also some aspects of emotional engagement in the construct identified by this thesis; in particular 'I am willing to put in effort beyond that normally expected in order to help the NZDF be successful'. There is also one item which can be considered normative (refers to commitment based on feelings of personal obligation and a sense of loyalty) rather than affective commitment: "I think I am doing something worthwhile for my country by being in the NZDF."

Normative commitment and affective commitment are typically highly correlated and this correlation increases in more hierarchical contexts (Fischer \& Mansell, 2009). The military would be one such context. Additionally, in the military, obligation, duty and service are key drivers of personnel's choice to join and stay with the military. Therefore, particularly for this organisation the two types of commitment are highly related.

A key difference between affective organisational commitment and engagement is the referent; affective organisational commitment refers to attachment to the organisation whereas engagement refers to cognitive, emotional and physical involvement in the work itself (Saks, 2006). As the entity referred to throughout these items is the NZDF, affective commitment is a more appropriate name for the 
scale. Additionally, the construct in this thesis shares 5 items with the Winsborough, Morris and Hughes (2008) scale 'military belonging'.

\section{Leaving Intentions}

\section{$\underline{\text { Improvements in Fit }}$}

Leaving intentions also showed poor fit. The RMSEA is of concern as it indicates that there is sampling error associated with this sample. The item 'it would take very little change in my current circumstances to cause me to leave the NZDF' is conceptually different to the other items. The other items refer directly to intentions towards staying or leaving NZDF and this item refers to a change in circumstances which is implied by the items to be related to intentions to stay or leave, however this may not be the case. This item was also the lowest loading item in the EFA. The fit statistics detailed in table 6 indicate that this change improves fit to acceptable levels.

\section{$\underline{\text { Content Validity }}$}

The intent to leave construct identified in this thesis contains items which measure both behavioural intent to leave, and likelihood of remaining over a certain time period. In comparison, Willness, Steel and Lee (2007) reported the use of three measures of job withdrawal or turnover intentions in their meta-analysis. All identified studies of work withdrawal used the either the Job Withdrawal Scale (Hanisch \& Hulin, 1990), the Michigan Organisational Assessment Questionnaire (MOAQ; Cammann, Fichman, Jenkins \& Klesh, (1983) or the Staying or Leaving Index (SOLI; Larson, Lakin, \& Bruininks, 1998).

The Job Withdrawal Scale examines intentions to quit, thinking about quitting, and perceived difficulty in securing alternative employment (Hanisch \& Hulin, 1990). The MOAQ intent to turnover measure contains items such as 'I will probably look for a new job in the next year.' 'I often think about quitting' and 'how likely is it that you could find a job with another employer with about the same pay and benefits you now have?' (Cammann, Fichman, Jenkins \& Klesh, 1983). The SOLI contains 4 items that asses the likelihood of leaving the organisation over different time periods, and 4 items that assess the likelihood of remaining during the same time periods 
(Larson, Lakin, \& Bruininks, 1998). Therefore the leaving intentions construct identified in this thesis compares well to those used in the literature.

\section{Summary}

The development and validation of the measurement model using EFA and CFA procedures in two samples resulted in nine psychometrically and conceptually valid constructs which can be used to test the structural model of the relationships between the antecedents and consequences of WIM, as detailed by hypotheses 1-12. 


\section{STUDY 2: STRUCTURAL MODEL}

The first test of the structural model involves a regression of the WIM construct on the hypothesised predictors using the data from the first sample. Informed by the results of the regression, and the relevant literature, a structural model is developed and tested using a second sample. The regression is reported first, followed by the SEM.

\section{Regression}

\section{Analysis}

In order to find empirical support for the hypothesis antecedents a regression of the antecedents hypothesised to predict WIM was conducted using the 2008 data (sample 1). Regression cannot account for the error of the measurement model as structural equation modelling can. However, regression is useful and gives an indication of whether the hypothesised antecedents are related to WIM. The regression was informed by the PCA conducted in the first part of study 1 (as detailed in figure 4) and therefore uses composite variables that reflect the factors in the PCA. The results of the regression are displayed in table 11. Constructs that were entered into the regression model as predictors were:

Equity and Diversity Climate (mean score across 4 items),

Aligned-Cohesive Culture (mean score across 12 items),

Directive Leadership (mean score across 11 items), and

Health and Safety Climate (mean score across 6 items).

In addition, the following demographic variables were included:

Age,

Gender,

Service (coded as a dummy variable with Civilians as the baseline),

Ethnicity (coded as a dummy variable with NZer/Paheka as baseline), and

Seniority (measured by income: last annual pay). 
Income (last annual pay) is included as a proxy for seniority (instead of rank). Although rank is an intuitively appealing variable for determining relative power in a military sample, two issues are problematic when using rank data. First, if rank data were included all civilian employees would be excluded from the model. The number of cases excluded would be 1570; of this number 1118 are civilians. This data is therefore not missing at random. This thesis aims to identify the predictors of WIM for all employees in the military environment, including civilians. Second, military ranks are organised into two parallel streams: commissioned officers, and noncommissioned service personnel. All commissioned officers, even junior ranks, are technically superiors of all non-commissioned service personnel, in that the non-commissioned service personnel must obey any lawful command of any officer. However, in practice, the two groups work together, e.g. a senior officer and senior non-commissioned soldier form a command team and a junior officer would rarely command a senior sergeant to do anything if they wanted to maintain the respect of the troops. If the technical rank structure was used to represent power it would represent a distortion. Instead pay is used as it allows for overlap of the two parallel rank streams. Pay most accurately reflects the seniority and influence held by personnel within NZDF.

\section{Results}

Table 11: Predicting WIM

\begin{tabular}{lccc}
\hline & & & \\
& $B$ & $S E B$ & $\beta$ \\
\hline Constant) & 5.326 & .097 & $-.211^{* *}$ \\
Leadership & -.259 & .019 & $-.174^{* *}$ \\
Equity \& Diversity Climate & -.241 & .022 & $-.112^{* *}$ \\
Aligned-Cohesive Culture & -.158 & .023 & $-.098^{* *}$ \\
Income & $-3.905^{-6}$ & .000 & $.072^{* *}$ \\
Service: Civilian vs. Army & .136 & .038 & $.071^{* *}$ \\
Service: Civilian vs. Navy & .164 & .043 & $.068^{* *}$ \\
Gender (male = 0, female $=1$ ) & .135 & .030 & $-.055^{* *}$ \\
Health and Safety & -.091 & .026 & $-.038^{*}$ \\
Age & -.003 & .001 & .004 \\
Service: Civilian vs. Air Force & .007 & .039 & .002 \\
Ethnicity: NZ European/Pakeha vs. Maori & .006 & .039 & .004 \\
Ethnicity: NZ European/Pakeha vs. Other & .009 & .031 & .052 \\
Ethnicity: NZ European/Pakeha vs. Not Stated & .037 & & .010 \\
\hline
\end{tabular}

Note $R^{2}=.204 * * p<.001, * p<.05$. Method: enter, $\mathrm{n}=4261$ listwise deletion 
Correlations between predictors are low (highest is $r=.453$ for age and pay) therefore multicollinearity is not an issue. The model would likely generalise well to the population as the adjusted $R^{2}=.201$ is very similar to the $R^{2}$. However, the model accounts for only $20 \%$ of the variance in WIM.

Of the organisational constructs hypothesised to predict WIM equity climate, aligned-cohesive culture, leadership and health and safety all protect against WIM. Army and Navy personnel, younger people, lower paid people, and females report more WIM. None of the ethnicity comparisons had significant beta values. 


\section{Structural Equation Model}

This thesis aims to develop and test a structural model of the antecedents and consequences of WIM using the second sample used for the CFA in study 1.

Relationships in the structural model are based on two sources. First, the theoretical hypotheses (1-11) generated by the literature and second, the empirical regression results reported conducted on the first sample.

Hypothesis 1: Aligned-cohesive culture will be negatively related to WIM.

Hypothesis 2: Equity and diversity climate will be negatively related to WIM..............................................................24

Hypothesis 3: Health and safety climate will be negatively related to WIM..................................................................25

Hypothesis 4: Army will demonstrate more WIM, compared with civilians, than either Navy or Air Force...................26

Hypothesis 5: Directive leadership will be negatively related to WIM. ..........................................................................2 28

Hypothesis 6: Seniority will be negatively related to WIM...............................................................................................29

Hypothesis 7: Females will report more WIM compared to males. .................................................................................29

Hypothesis 8: Ethnic minorities will be report more WIM compared to the ethnic majority (NZ Europeans) ............... 30

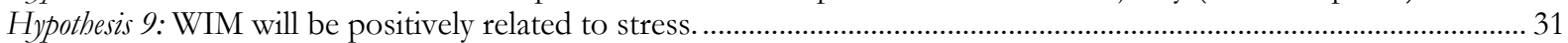

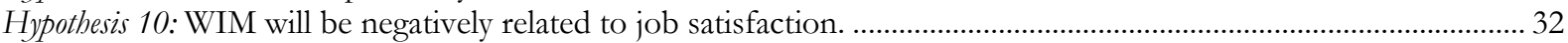

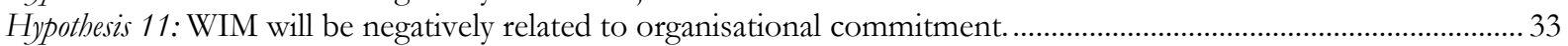

Hypothesis 12: WIM will be positively related to leaving intentions............................................................................ 33

As the CFA in study 1 identified that leadership is best represented by two constructs:

'Leadership' and 'Trust in Supervisor', an additional antecedent is also included in the model.

The regression in study 1 , which used a different sample and can therefore, be used to inform this study, found that personnel in the Navy and Army, younger personnel, lower paid personnel, and females report more WIM. Equity climate, aligned-cohesive culture, perceived supervisor support, and health and safety were all found to protect against WIM. The findings from the regression in study 1 may not be replicated as some of the predictors have been modified where items were removed due to CFA results and additionally SEM uses different techniques to regression, specifically, the measurement error is controlled.

\section{Analysis}

The approach used for this thesis is Joreskog's (1993) Model Generating approach. A tentative model (Model A) was developed based on the literature and tested and modified as necessary. As 
the focus of this thesis is to identify the antecedents and consequences of WIM the first model tested was a simple model based on the hypotheses 1-12 with the addition of the 'Trust in Supervisor' construct as a predictor. In this model all antecedents directly predict WIM and all consequences are directly predicted by WIM. This results in a fully mediated model (model A).

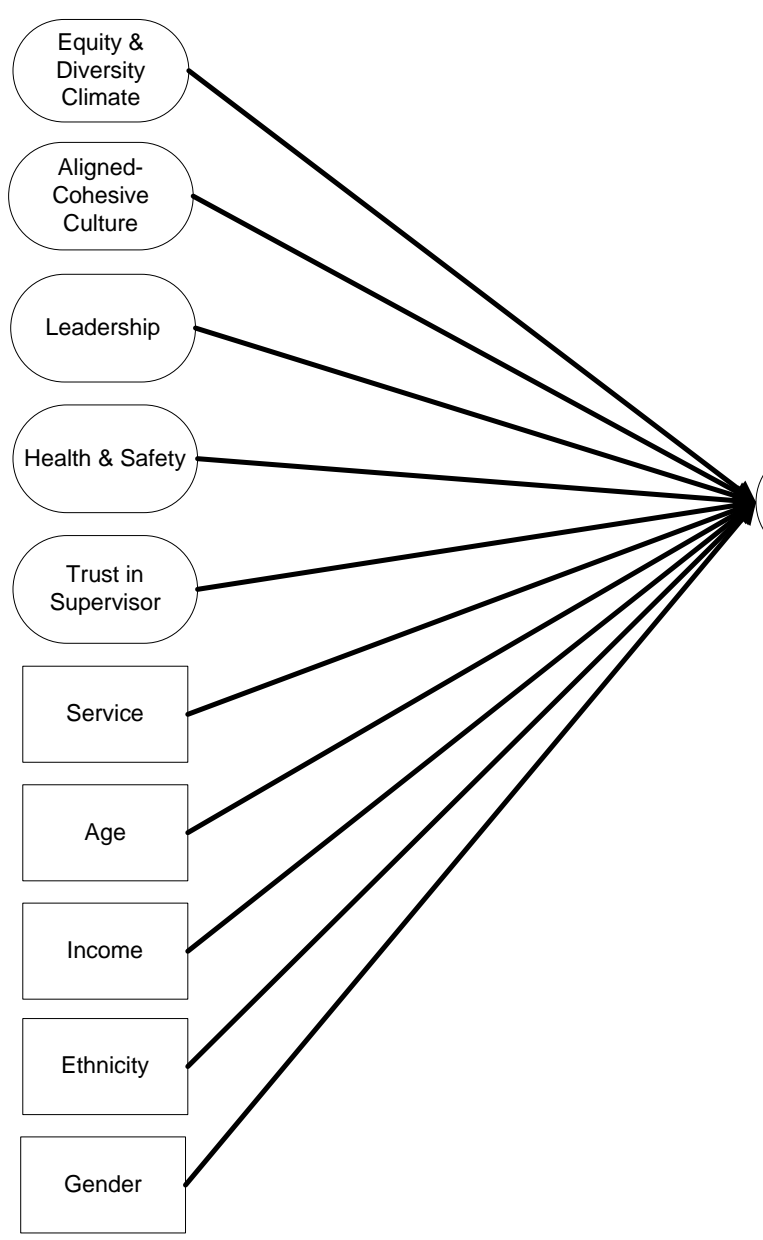

Model A WIM

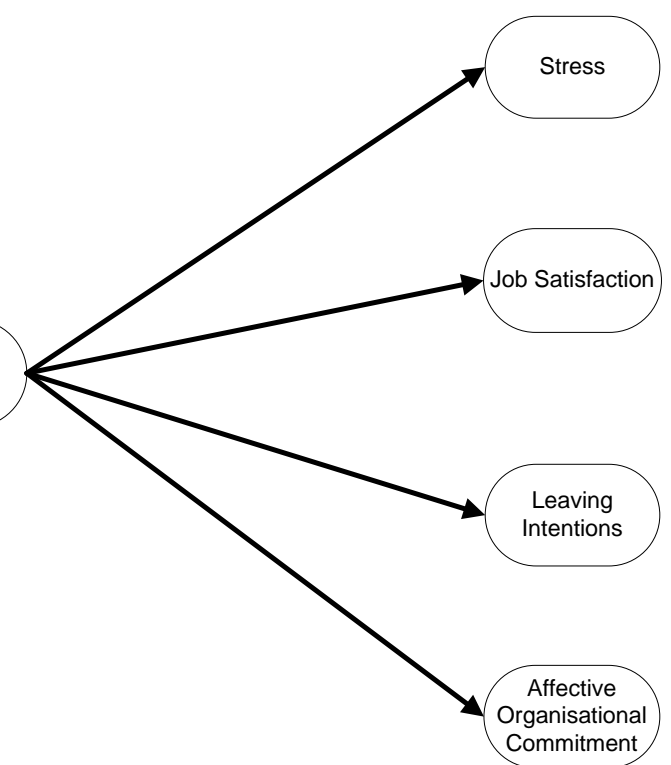

Figure 8: Hypothesised model A: fully mediated model

The Model Generating approach is the most common approach used by researchers and a key consideration when using this approach is that any modifications made must not be driven solely by the statistical fit to data but also by theory. If modifications are guided solely by the statistical fit to the data then the model is unlikely to generalise. Alternative models are tested using the same data until a model that makes conceptual sense and has adequate fit is identified.

Items were parcelled so that any construct had no more than 4 indicators. The items reflecting the WIM construct was not parcelled due to the complex structure of this construct. Item parcelling is a controversial technique (Cunningham, 2008) but it is acceptable in this case as the un-parcelled measurement model has already been demonstrated to have acceptable fit using CFA. The main goal of parcelling is to reduce the number of measured variables to within the 35 
limit recommended by (Marsh, Hau, \& Wen, 2004). Items were parcelled randomly. Dummy variables were created to represent categorical variables with more than two categories (service and ethnicity).

The dataset used is sample 2; figure 4 indicates that this is the same sample that was used in the CFA in study 1. This approach is acceptable for the model generating phase, as any models developed will be cross-validated in the final hold out sample. 163 further cases were removed from the dataset due to missing demographic data.

\section{Results}

Fit indices indicate that Model A fits the data reasonably well: $x^{2}(769)=5135.767, p=.001$, RMSEA $=.053, \mathrm{TLI}=.889, \mathrm{CFI}=.907$, and SRMR $=.0898$, but the fit for SRMR and TLI could be improved. 
Table 12: Standardised B coefficients for antecedents and consequences of WIM, model A

\begin{tabular}{lccc}
\hline & Antecedents & Consequences \\
\hline Equity \& Diversity Climate & $-.139^{* * *}$ & \\
Health \& Safety Climate & $-.187^{* * *}$ & \\
Directive Leadership & $-.170^{* * *}$ & \\
Trust in Supervisor & -.022 & \\
Aligned-Cohesive Culture & $-.205^{* * *}$ & \\
Age & -.018 & \\
Gender & $.087^{* * *}$ & \\
Income & $-.141^{* * *}$ & \\
Civilians vs Navy & .055 & \\
Civilians vs Army & $.080^{*}$ & \\
Civilians vs Air Force & .023 & \\
NZ Pakeha vs Maori & -.023 & $.407^{* * *}$ \\
NZ Pakeha vs Other & .038 & $-.453^{* * *}$ \\
NZ Pakeha vs Not Stated & -.018 & $-.440^{* * *}$ \\
Stress & & $.385^{* * *}$ \\
Job Satisfaction & & \\
Organisational Commitment & & \\
Leaving Intentions & & \\
\hline Not & & \\
\hline
\end{tabular}

Note: standardised B coefficients shown, ${ }^{* * *} p<.001,{ }^{* *} p<.01, * x<.05$.

All antecedents and consequences are significantly related to WIM in the direction predicted by the hypotheses with 4 exceptions. Trust in supervisor is not significantly related to WIM. Only Army personnel show significantly more WIM than civilian personnel. Age is not significantly related to WIM and neither is ethnicity. The non-significance of the relationships between ethnicity and WIM replicates the result found in the regression. The non-significance of age and Navy as predictors of WIM is in contrast with the results of the regression analysis. Trust in supervisor was not measured separately in the regression and therefore was not studied.

However, as the model fit could be improved some model re-specification was necessary.

\section{Model Respecification}

The model was re-specified to allow for direct paths between some antecedents and consequences, based on modification indices. Specifically, Leadership is related to Job satisfaction via trust in supervisor and aligned-cohesive culture is related directly to affective organisational

${ }^{11}$ Coded males $=0$, females $=1$ 
commitment. Antecedents which were not associated with WIM (Age and Ethnicity) were removed. In addition, a more complex series of relationships between the outcomes of WIM were modelled where stress, job satisfaction and affective organisational commitment mediate the relationship between WIM and leaving intentions. The re-specified model (Model B) shows good fit: $\chi^{2}(623)=2761.058, p=.001$, RMSEA $=.042$, TLI $=.944$, CFI $=.951$, and SRMR $=.0483$.

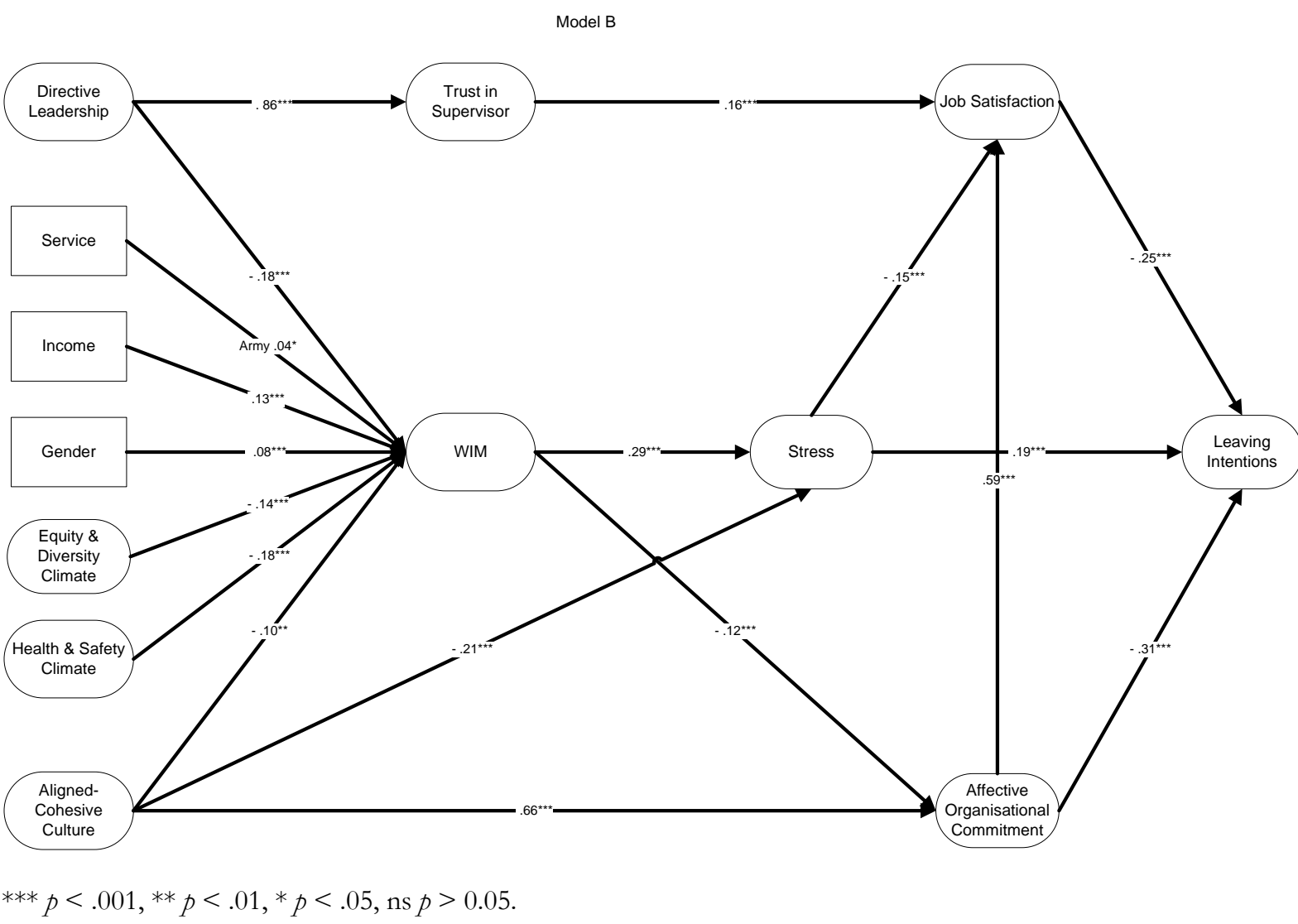

Figure 9: Standardised B coefficients for Model B sample 2 (2007)

Model B explains variance in endogenous constructs ranging from $17 \%$ for stress to $74 \%$ for trust in supervisor. 
Table 13: Variance in endogenous constructs explained by Model B (sample 2, 2007 data)

Construct

Variance explained by model (\%)

WIM

25

Trust in Supervisor

74

Job Satisfaction

50

Stress

17

Affective Organisational Commitment

50

Leaving Intentions

36 


\section{Discussion}

\section{Regression}

The regression analyses support the hypothesised relationships between all organisational variables and WIM. Directive leadership was found to be the best predictor of WIM. This is indicative of the important role that leaders in the military have for their subordinates. The next two most important predictors were equity and diversity climate and aligned-cohesive culture, both which describe the nature of the work environment although equity and diversity climate is measured at the workplace level and aligned-cohesive culture is measured at the organisational level. Equity and diversity climate was found to be the second best predictor of WIM even though the measure used in this thesis is a relatively more distal measure of equity and diversity climate compared to those typically used in the literature.

Another feature of the work environment - health and safety climate was also a significant predictor but this did not make as much of a contribution as the other organisational variables. This may be due to the nature of the health and safety measure which addresses an issue which is only peripherally related to WIM.

The relationships between the individual variables and WIM did not consistently support the hypotheses. In particular ethnicity is not significantly related to WIM. This is interesting given that some of the WIM items refer specifically to discrimination under the Human Rights Act. The NZ military has a strong Maori tradition and culture. This may explain why Maori did not report significantly more WIM than their New Zealander/Pakeha counterparts. The 'other' ethnicity group was comprised of all other ethnic minorities. It may be that this grouping of both European (e.g. Irish, Scandinavian) with other non-European ethnicities masked the effect of the non-European (and therefore more identifiably minority) ethnicities on WIM. This grouping was necessary due to the small numbers making up the non-Maori, non-New Zealander/Pakeha ethnic groups.

Age was less of a predictor than income which was the other seniority-related variable. This is consistent with the nature of the military environment discussed earlier which contains two parallel rank structures which military personnel promote through. This complex system results in a young group of officers with a relatively large amount of influence and another group of 
older soldiers with a relatively smaller amount of influence considering their age. This result confirms that income is the simplest way to conceptualise seniority in the military environment.

The regression technique used in this sample does not account for measurement error. Therefore, it was important to replicate these findings using SEM techniques in Study 2 and 3. Additionally, consequences of WIM (rather than just antecedents) were investigated which were not assessed in this regression.

\section{Model A}

Regression weights indicate that equity and diversity climate, health and safety, leadership, gender, income, aligned-cohesive culture, and being in the Army all predict WIM. These relationships are in the predicted directions where a better equity and diversity climate, health and safety climate, leadership and aligned-cohesive culture are all related to less WIM, and females, Army personnel and personnel who are paid less report more WIM. Personnel who report more WIM also report more stress, lower job satisfaction and organisational commitment and increased leaving intentions. The model can account for 35\% of the variance in WIM. The model can also account for $17 \%$ of stress, $21 \%$ of job satisfaction, $15 \%$ of leaving intentions and $19 \%$ of affective organisational commitment. However, the model did not fit the data that well and therefore, these results should be interpreted with caution.

\section{Model B}

Some significant respecification occurred to create model B which resulted in a model that fits the data better. However, a significant consideration of Joreskog's (1993) model generating approach is the requirement for the researcher to provide adequate justification and interpretation for modifications to models. Justification and interpretation is provided below for features of the model which were re-specified.

\section{$\underline{\text { Service Culture }}$}

Army was found to be the only service in which personnel report significantly more WIM than civilians. Previously in model A and the regression Navy personnel also reported significantly more WIM than civilians. A hierarchy may exist where the cultures of the Army, then Navy, then Air Force are more different from civilian culture. Army is characterised as more competitive, 
combative, and interpersonally adversarial than the other services. For example, in NZ, the Army is the only service which includes close quarter combat training (hand-to-hand combat training with the intent to kill the opponent) as part of physical training (NZ Army News, August 2011). These features of Army culture may result in an environment which is characterised by more WIM. Additionally, organisations where there is an extreme degree of conformity and group pressure are prone to WIM (Hoel \& Salin, 2003). As Army personnel provide value in relation to their team, rather than in relation to a piece of technical equipment (such as a ship or aircraft), this may result in a culture where conformity and group pressure are more intense. Work undertaken in stressful environments such as extremes of noise and temperature or in unpleasant conditions has been found to be associated with increasingly hostile attitudes (Anderson, Anderson, \& Deuser, 1996; Einarsen \& Skogstad, 1996). Both Army and Navy personnel undertake work in extreme environments over long periods of time either in the field or on a ship. In contrast, the extreme environments experienced by Air Force personnel tend to occur for shorter periods (i.e. the duration of a flight). Civilians are not routinely exposed to extreme environments.

\section{$\underline{\text { WIM Outcomes }}$}

The outcomes of WIM are modelled in a more complex way in Model B. This is to account for the mediating role that stress, affective organisational commitment and job satisfaction play in the relationship between WIM and leaving intentions. This revised model fits well with a number of organizational theories and empirical findings.

First, focusing on the mediation relationship of stress in the relationship between WIM and leaving intentions, existing work on the outcomes of WIM has suggested that stress is an outcome of WIM (Lim and Cortina, 2005) for both targets and observers (Vartia, 2001) and that stress mediates the relationship between WIM and leaving intentions in nurses (Dion, 2006). In accordance with this, in the current study the relationship between WIM and leaving intentions is mediated by stress.

Prior work on the relationship between stress and leaving intentions has shown that the effect of stress on leaving intentions is mediated by job satisfaction (Williams et al. 2001). In line with this, in the current data the relationship between stress and leaving intentions is mediated by job satisfaction (in addition to the direct path from stress to leaving intentions). 
The second relationship of interest is the mediating role of organisational commitment in the relationship between WIM and leaving intentions. Prior work on the role of organisational commitment has indicated that organisational commitment mediates the relationship between bullying and harassment on leaving intentions in restaurant employees (Mathisen, Einarsen \& Mykletun, 2008). In accordance with this, in the current study the relationship between WIM and leaving intentions is mediated by organisational commitment.

The importance of job satisfaction and organisational commitment on leaving intentions has been empirically demonstrated in research in the NZ military (Winsborough, Morris, \& Hughes, 2008). The authors found that job satisfaction and military belonging (which was measured by 8 of the items in the affective organisational commitment in this thesis) predicted intention to leave via 'Anomie' or a state where personnel have 'checked out' of the organisation and are waiting for an external opportunity to present itself before leaving. This state was also highly predictive of actual leaving behaviour. Therefore, models which predict leaving intentions as a key outcome such as the model of antecedents and consequences of WIM in the current study should account for impact of affective organisational commitment and job satisfaction on leaving intentions directly. Model B reflects these predictors of WIM.

In the final model organisational commitment predicts job satisfaction but the reverse relationship was not included (as it did not improve fit). In the literature, there is much discussion about the directionality of the relationship (e.g. Rayton, 2006; Curry, Wakefield, Price \& Mueller, 1986). The reason for the direction of this relationship in the current study relates to the specific features of the sample used. Military personnel commit to the organisation (their service) rather than their job. The nature of military work requires the commitment to something more permanent than the job as military personnel cycle through many postings (jobs) during their career. Military personnel expect that they will not enjoy some or many of these jobs. Manning (1994) argues that for military personnel organisational commitment is more stable "in the face of day to day work experiences" (p6) than job satisfaction and that organisational commitment is more global, and develops and dissipates more slowly. As long as their belief in the aims of the organisation continue military personnel are likely to feel reasonably satisfied in a given job. However, if their commitment to the organisation is undermined their satisfaction with their job is also likely to be undermined. As it is almost impossible to feel satisfied with your work if you do not believe in or belong to the greater organisation. Therefore, in model B organisational 
commitment predicts job satisfaction, but job satisfaction does not predict organisational commitment.

These organisational theories suggest that WIM is unlikely to cause personnel to consider leaving the organisation on its own but instead that the benefits that keep people in their jobs, such as enjoying one's work and feeling committed are undermined, and costs such as stress are increased. These factors are the mediating psychological mechanism by which people start to consider leaving.

\section{Direct Effects of Antecedents on Outcomes}

Additional direct paths from predictors to outcomes are also included in Model B. Alignedcohesive culture is directly related to affective organisational commitment and leadership predicts job satisfaction. The reason for these specific relationships and not the vice versa (e.g. alignedcohesive culture predicting job satisfaction) is the level of measurement. As aligned-cohesive culture is a characteristic of the organisation, not the individual or team, it is associated with outcomes which are also related to the organisation such as organisational commitment (Beehr, Glazer, Fischer, Linton \& Hansen, 2009)

The referent for items in the aligned-cohesive culture construct is the NZDF or service, the referent for items in the leadership construct is the immediate supervisor. The referent for the related outcome measures relates to their predictors; for affective organisation commitment the referent is the organisation and for job satisfaction, the referent is the work and job which is primarily determined by the supervisor. Therefore, the direct effects in the model are constrained to a single level of measurement. Constructs which refer to the workplace are related (directive leadership predicts job satisfaction) and constructs which refer to the organisation are related (aligned-cohesive culture predicts organisational commitment).

Trust in supervisor moves from a predictor of WIM to a mediator of the relationship between leadership and job satisfaction. Trust in supervisor is an evaluation of the leader based on the performance of their leadership responsibilities. The process of evaluation of the leader as trustworthy and deserving of respect increases the legitimacy of the work that the leader tasks personnel with. Increased legitimacy of the work results in increased job satisfaction as belief that 
the work one undertakes is important creates meaning in the performance of the job and intrinsic satisfaction. Therefore trust in supervisor acts as a lens through which job satisfaction increases. Alternatively, if poor leadership results in an evaluation of the leader as untrustworthy or not deserving of respect then this undermines what might otherwise be an enjoyable job, and therefore undermines job satisfaction.

One relationship in the model which does not conform to the separation of organisational antecedents predicting organisational outcomes and immediate workplace antecedents predicting workplace outcomes is the effect of aligned-cohesive culture on stress. This is due to unique features of the organisation studied in this thesis. Arguments made earlier in this thesis for the effect of organisational commitment on job satisfaction also apply to the effect of alignedcohesive culture on stress. That is, personnel who join the military do so as an act of service to the particular service they join (Navy, Army or Air Force). They do not commit to a specific job within that service. The specific job that personnel undertake at any one time is transitory. The service is the constant. Therefore, the undermining of important features of military culture (alignment and cohesion) results in stress, whereas the effects of poor leadership are not significantly related to stress. If personnel are especially committed to the organisation and have undertaken a great deal of personal sacrifice for the good of the organisation, the experience of an undermined culture is likely to be very stressful. Personnel may perceive that their commitment and loyalty is not returned by the organisation and may question whether the personal sacrifices were worthwhile. Therefore, military personnel are perhaps more resilient to the stress effects of poor leadership as they can rationalise the situation as only temporary. They may also be uniquely vulnerable to the effects of poor aligned-cohesive culture as this undermines their sense of place in the organisation. This result was not reflected in the regression analysis conducted on sample 1 which revealed directive leadership had a strong influence on WIM. However, two differences may explain this discrepancy. First, stress was not measured in the regression as it is hypothesised to be an outcome of WIM rather than a predictor and therefore the regression did not assess the relationship between directive leadership and stress. Second, the directive leadership construct used in the regression included 4 extra items which in the SEM analysis are not included.

The inclusion of these direct relationships on outcomes reduces the importance of WIM in the model, but improves model fit. Inclusion of direct paths mean that the unique effects of WIM on 
organisational outcomes are more robustly modelled, after controlling for the effects of important predictors directly on the outcomes. 


\section{STUDY 3: VALIDATION OF THE STRUCTURAL MODEL}

Study 3 aims to validate model B identified in study 2 using a different sample of data in order to demonstrate replicability and to provide an indication of the whether the results will generalise.

\section{Analysis}

The third sample which has not been used in any of the previous studies is used for this study. The use of a fresh sample allows for the validation of the structural model. In this sample 480 cases were removed due to missing data in either demographic variables or relevant parcels or constructs. After missing data was removed there is a sample size of 3182 cases. Model B was analysed exactly as modelled in study 2 but with the 2009 dataset. The same item parcels were used as in study 2. 


\section{Results}

Table 14: Descriptive statistics and correlation among the study variables for sample 3

\begin{tabular}{|c|c|c|c|c|c|c|c|c|c|c|c|c|}
\hline & & Mean & $\mathrm{SD}$ & 1 & 2 & 3 & 4 & 5 & 6 & 7 & 8 & 9 \\
\hline 1 & Directive Leadership & 3.8086 & 0.69528 & & & & & & & & & \\
\hline 2 & Trust in Supervisor & 3.9643 & 0.87431 & $.791 * *$ & & & & & & & & \\
\hline 3 & Organisational Commitment & 4.1000 & 0.50374 & $.330^{* *}$ & $.304 * *$ & & & & & & & \\
\hline 4 & Aligned-Cohesive Culture & 3.5913 & 0.59015 & $.425^{* *}$ & $.353^{* *}$ & $.601 * *$ & & & & & & \\
\hline 5 & WIM & 2.1861 & 0.85931 & $-.337 * *$ & $-.307 * *$ & $-.297 * *$ & $-.340^{* *}$ & & & & & \\
\hline 6 & Job Satisfaction & 3.7862 & 0.68500 & $.380^{* *}$ & $.332^{* *}$ & $.556^{* *}$ & $.460 * *$ & $-.292^{* *}$ & & & & \\
\hline 7 & Health and Safety & 4.0263 & 0.52578 & $.344^{* *}$ & $.267 * *$ & $.339 * *$ & $.365^{* *}$ & $-.269 * *$ & $.304 * *$ & & & \\
\hline 8 & Stress & 2.4630 & 0.78568 & $-.246^{* *}$ & $-.209 * *$ & $-.237 * *$ & $-.319 * *$ & $.322 * *$ & $-.270 * *$ & $-.212 * *$ & & \\
\hline 9 & Equity and Diversity Climate & 3.6888 & 0.61790 & $.276^{* *}$ & $.213^{* *}$ & $.364^{* *}$ & $.395^{* *}$ & $-.331 * *$ & $.280^{* *}$ & $.425^{* *}$ & $-.183^{* *}$ & \\
\hline 10 & Leaving Intentions & 2.4451 & 0.98388 & $-.291 * *$ & $-.286^{* *}$ & $-.471 * *$ & $-.452^{* *}$ & $.276^{* *}$ & $-.488^{* *}$ & $-.201 * *$ & $.355^{* *}$ & $-.210^{* *}$ \\
\hline
\end{tabular}

**. Correlation is significant at the 0.01 level (2-tailed).

Summary statistics for sample 3 are displayed in table 14. Mean scores are quite high for many constructs, with the exception of WIM and stress which are at or just below average, as was the case with samples 1 and 2 . The variation in scores (SD) are highest for leaving intentions although in the $3^{\text {rd }}$ sample the SD does not reach 1. As with samples 1 and 2 there are a number of sizable correlations among constructs with strongest relationships between Directive leadership and trust in supervisor, and aligned-cohesive culture and organisational commitment. Given the conceptual closeness of these constructs, the magnitude of these relationships are to be expected and are not unusual. 
Model B showed good fit in the 2009 data: $\chi^{2}(623)=4021.397, p=.001$, RMSEA $=.041$, TLI $=$ $.947, \mathrm{CFI}=.953$, and SRMR $=.04503$. The amount of variance in the endogenous variables explained by the model is similar to that found in study 2. Beta weights were also similar to those found in study 2 with one exception: gender was found to be non-significant in this sample.

Table 15: Variance in endogenous constructs explained by the model (sample 3, 2009 data)

Construct

Variance explained by model (\%)

WIM

Trust in Supervisor

Job Satisfaction

Stress

Affective Organisational Commitment

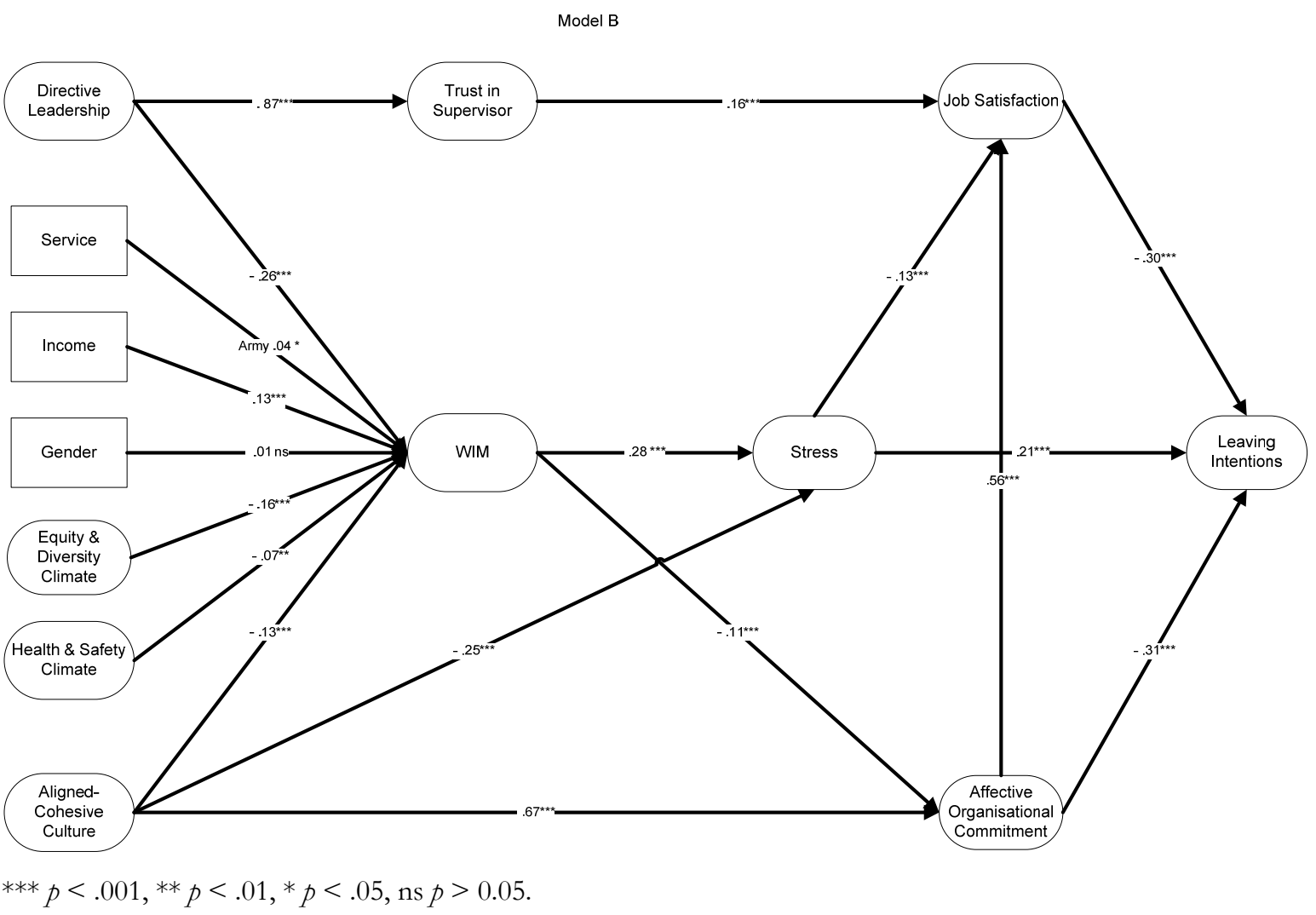

Figure 10: Relationships among constructs in Model B, sample 3, 2009 


\section{Discussion}

Generally the model holds and shows good fit in both samples. This suggests it will generalise well to other samples in the population. All indicators (parcels and items) are significant reflectors of their latent constructs.

\section{Differences between the Two Samples}

The main difference between the two samples is that gender goes from being a significant predictor of WIM in the sample 2 model $(<0.001)$ to a non-significant predictor in the sample 3 $(\mathrm{p}=0.453)$. The $\mathrm{p}$ value in the $3^{\text {rd }}$ sample is convincingly non-significant and represents an important change. There are about the same proportion of females in the different year groups; $23 \%$ in 2007 and $26 \%$ in 2009 .

Table 16: Proportion of females in samples 2 and 3

\begin{tabular}{llcc}
\hline & & Frequency & Percent \\
\hline Sample 2 & Male & 1469 & 76.6 \\
& Female & 450 & 23.4 \\
Sample 3 & Male & 2357 & 74.1 \\
& Female & 825 & 25.9 \\
\hline
\end{tabular}

This change is difficult to explain as although many studies have identified gender differences in reported rates of WIM, very little theorising or in-depth investigation into the reasons for these differences has been done (Zapf, Einarsen, Hoel \& Varita, 2003). The non-significant result for gender in the second sample is part of a trend towards the reduction in importance of individual level variables. The regression analysis showed a significant contribution for four individual level variables: gender, income, and Army and Navy compared to civilians. Navy was no longer a significant predictor when SEM was conducted, and here in the validation sample, gender also becomes non-significant. From the original list of individual level variables only income is left as a protective factor, and being in the Army as a risk factor.

Other changes noted in the validation sample reflect changes in magnitude in the relationships rather than changes of significance or direction. The changes in magnitude include an increase in the relationship between job satisfaction and leaving intentions, a decrease in the impact of health and safety climate on WIM, and an increase in the influence of aligned-cohesive culture on stress. 


\section{DISCUSSION}

This thesis makes three important contributions to our understanding of WIM. The first is to improve our understanding of the structure of the WIM construct itself. The second is to demonstrate support for a theoretically plausible model of the antecedents and consequences of WIM. The third is of more practical consideration which is that it determines the structure of constructs measured by a practitioner developed attitude survey.

\section{WIM Construct}

The WIM construct has a complex structure: there are both commonalities underlying a diverse range of WIM behaviours, and the observation of multiple types of WIM is more closely related than the experience of different types of WIM. The structure of the WIM construct suggests that observed WIM may reflect a dysfunctional work environment. In contrast, experienced WIM behaviours (harassment, bullying and discrimination) do not necessarily co-occur and may reflect different experiences based on membership to demographic groups. This thesis addresses Willness, Steel and Lee's (2007) calls for further psychometric work on the WIM construct. This thesis also contributes to the debate about the nature of WIM, which has historically produced mixed results. Some studies have identified a single construct underlying wide ranging behaviours (e.g., Griffin, 2009) and others have identified a more complex structure (e.g., Raver \& Nishii, 2010). This thesis is the first study to use structure-oriented techniques (factor analysis) to assess the WIM construct across observed and experienced WIM. Previous research that has considered observed and experienced WIM simultaneously has taken the approach of comparing the outcomes of observers and targets, although it has been acknowledged that people can be members of both groups (e.g. Cooper-Thomas, Catley, Bentley, Gardner, O’Driscoll, \& Trenberth, 2011). Therefore this thesis provides a broadly defined psychometrically valid measure of WIM.

The inclusion of both observers and targets of WIM, and the discovery that observed WIM may reflect a dysfunctional work environment. This idea raises questions around the multi-level nature of WIM. The first question would be whether observed WIM can function as a group level construct i.e. is there consensus of observed WIM within each work group. If consensus is established, additional questions such as how does a dysfunctional work environment influence experienced WIM and how does experienced WIM influence the functionality of the work 
environment? Is the process primarily bottom up or top down? A multilevel modeling approach could answer these questions. I speculate that that the process is initially top down, in that a dysfunctional work environment at the group level influences reported experienced WIM, but that once this occurs reported WIM also influences the functionality of the work environment. Testing of this hypothesis would require multi-level techniques in a longitudinal study.

\section{Model of Antecedents and Consequences}

The theoretically plausible model tested in this thesis addresses a significant gap in the literature as it covers both the antecedents and consequences of WIM and identified these for a broad definition of WIM that includes three different behaviours. This thesis focused on predictors which are primarily under an organisation's control and therefore represent an opportunity to bring about practical change. However, some demographic variables were included in the model. The demographic predictors of WIM were not found to be as important as the organisational variables. This finding supports Monks et al.'s (2009) assertion that the primary responsibility for change lies with the organisation. The findings also suggest that management can have an impact on the rates of WIM in their organisation. However, the model only explains $25 \%$ of the variance in WIM so there are clearly other causes that are not covered by this model. These additional areas are important even if they are not easily controlled. Future research investigating the other possible predictors of WIM (such as rate of organisational change; Einarsen, 1999) to try and improve the amount of explained variance in WIM would be useful.

\section{Features of the Model}

Directive leadership is the strongest predictor in both models (although in sample 2 it is equal to Health and Safety Climate). This indicates that supervisor performance has a big role to play in protecting against WIM. This model suggests that if an organisation can improve supervisor performance of even basic directive leadership behaviour the incidents of reported WIM and associated negative outcomes are likely to reduce. This finding may be because supervisors have an influence on the workplace in multiple ways - supervisors can be perpetrators of WIM themselves, they can allow WIM to go unpunished between coworkers and they can also create a stressful work environment which is more likely to elicit WIM. An organisation would therefore obtain the most benefit from focusing attention on supervisor behaviour. If WIM is recognised as an important issue, preventing WIM, as measured by subordinates, could be reported on as part of supervisor's performance management. 
Health and safety climate is another predictor at the workplace level which has an influence on WIM. This finding is novel and extends our understanding of Health and Safety Climate's influence on WIM from just psychosocial health and safety climate (Law, Dollard, Tuckey, \& Dormann, 2011) to general Health and Safety Climate. This finding parallels the recent legislative changes in New Zealand which defines harm caused by WIM as a workplace hazard which organisations have a responsibility to prevent.

Equity and diversity climate is a significant predictor, but also less important than Directive Leadership. This suggests that personnel view WIM as a workplace issue, and that although it happens in the context of an organisation the workplace level predictors are more important. The two climate measures (equity and diversity climate and health and safety climate) make a modest impact on WIM, but steps to address the two issues by the organisation could be undertaken together. One such measure is education around what organisational climate is, the importance of climate and how to change climate.

Aligned-cohesive culture also has a modest impact on WIM. This finding suggests that personnel who perceive the organisation as aligned and cohesive report less WIM. Aligned-cohesive culture is a more remote construct than the leadership and climate measures already discussed, as it refers to the organisation. Aligned-cohesive culture also has a strong direct effect on organisational commitment. Therefore, it is in the organisations best interests for all personnel to experience an aligned and cohesive culture including features such as support from colleagues and superiors, and good communication throughout the organisation. Improving personnel's perceptions of such an environment would both decrease WIM and have positive effects on other variables desirable to the organisation; increased organisational commitment and decreased leaving intentions. However, these more general perceptions are difficult to build (Manning, 1994). Nevertheless, the benefits for the organisation are large and improvements in this area would be worth pursuing.

The second direct relationship from predictors to outcomes is the effect of directive leadership on job satisfaction via trust in supervisor. These direct effects reduce the importance of WIM in the model, but also provide more robust modelling of organisational outcomes as the effects of important predictors directly on the outcomes are controlled. Modelling the relationships in this way reflects multiple effects of constructs on one another, which more accurately reflects the real world. 
The final model shows a more complicated set of relationships for the outcomes of WIM than was originally hypothesised. The impacts of WIM on leaving intentions occur via its effect on stress and organisational commitment, though stress is a stronger outcome [what do you mean here?]. Stress in turn affects job satisfaction and leaving intentions. There is also a direct relationship between job satisfaction and organisational commitment. These more complex relationships reflect the complexity of the push-pull factors involved in attrition (Holt, Rehg, Lin, \& Miller, 2007).

The importance of stress in the modelling of outcomes is interesting as it offers support for Brosky's (1976) theory of WIM's influence on stress. The final model displays stress in relation to WIM in only one part of the model. However, the relationship between stress and WIM may be more complex. Bentley et al, (2009) propose that stress can be both a predictor and an outcome of WIM; yielding a recursive and cyclical relationship. Stress is proposed to predict WIM as a stressful work environment can elicit aggressive behaviour; experience of WIM is also a stressful experience (as modelled in this thesis). The recursive nature of this relationship would be a useful focus for further longitudinal studies as interrupting this cycle is important to prevent WIM escalating.

Demographic and individual level predictors were found to be less important than organisational predictors. In the final model only seniority and service were significant predictors of WIM, with seniority being the stronger predictor. These findings are in contrast to other studies (e.g., Zapf \& Einarsen, 2003) which have found demographic variables to be important predictors of WIM. This may be due to the inclusion of both observed and experienced WIM in the measure used in this thesis. Demographic variables will not be so clearly related to observed WIM as respondents can report on WIM that occurs to people who do not share their demographic. These results suggest that perceptions are more important than memberships to a specific demographic category. Einarsen, Hoel, Zapf and Copper (2003) have made a case that perception is at the heart of the matter of WIM, these results therefore support this assertion.

\section{Military Sample}

Although WIM in the military has been studied extensively in relation to sexual harassment (e.g., Magley, Waldo, Drasgow \& Fitzgerald, 1999; Williams, Fitzgerald, \& Drasgow, 1999) little research addressing WIM more generally has been done in the military, and almost none has been 
conducted in New Zealand (with the exception of Winsborough, Morris and Hughes, 2008). This thesis considers WIM in the New Zealand military context. Additionally, this is the first research which focuses on the protective influence that specific military culture can have on reducing WIM rather than just focussing on the negative features of the military environment (e.g. Ilies, Hauserman, Schochau \& Stibal, 2003). The findings demonstrate that there are influences of military culture that reduce WIM. This finding is encouraging as it offers a new positive perspective on the way that WIM occurs in the military and the approach the organisation can take to reduce WIM behaviours.

\section{Identifying Constructs}

Heeding calls by authors to use a collaborative research approach (e.g. Bartunek, 2007; Hinkin, Holtom, \& Klag, 2007; Anderson, Herriot \& Hodgkinson, 2001), this thesis used existing military data from an organisational attitude survey. Therefore, a practical contribution of this thesis is that it identified the constructs measured in the organisational survey in order to determine psychometrically valid measures of important organisational constructs including WIM and its antecedents and consequences. The constructs identified cover a broad range of areas important to organisational functioning:

1. Directive Leadership

2. Organisational Commitment

3. Aligned-Cohesive Culture

4. WIM

5. Job Satisfaction

6. Leaving Intentions

7. Health and Safety

8. Stress

9. Work Life Balance

10. Equity and Diversity Climate

11. Attitude to Change

12. Pay Fairness

13. Promotion

14. Work Quality Standards

15. Skills and Training

16. Performance Management 
17. Contribution

18. Employment Opportunities

19. Resourcing

Although both 'Employment Opportunities' and 'Resourcing' have only two items and therefore would not be useful as constructs in future models, the other 17 constructs have multiple items and show good reliability. The coverage of a diverse range of topics will allow the investigation of many different organisational issues in a robust way in future studies.

\section{Limitations}

One of the major limitations of this thesis is that it is cross sectional in nature and therefore causation cannot be inferred in this design. It is therefore possible that outcomes cause predictors rather than the other way around. One example of this already discussed is the relationship between stress and WIM. Future research should consider investigating these relationships using longitudinal or experimental approaches to identify causation. An associated issue is the influence of common method bias, as data for all measures was collected using a single tool and method. Although, Crampton and Wagner (1994) concluded, from meta-analytic work of self report measures in organisational research, that self-report measures are relatively robust and reliable indicators, especially if the ratings refer to perceptions of visible and external events that could easily be verified by external parties. Therefore common method bias is argued to be less of an issue.

Taking a collaborative research-inherit approach necessarily means that identification of the measures is a post-hoc exercise. Bergman et al. (2002) note that using scales which do not have a long psychometric history is less than ideal. Theoretical justification and empirical evidence was relied on to demonstrate that the constructs measured areas of interest and that the measures were comparable to established scales. However, the use of existing data also constitutes a strength of this thesis as it offers large samples across many areas of the organisation, and across a broad range of areas.

Although the sample size was large, the response rate was between $45-50 \%$. This figure is not unusual for research of this kind (Baruch \& Holtom, 2008) although it is less than ideal. The moderate response rate raises the question of how well the results will generalise to the population. This concern is mitigated by the use of multiple sample validation for both the measurement and structural parts of the model. No work has been done with the dataset used in 
this thesis to compare responders to non-responders. Future work could investigate this issue to provide evidence of whether these results will generalise.

\section{Future Research}

Future research directions already mentioned in the discussion fall into two broad areas: extension of the model and consideration of methodological issues. Potential improvements in modelling include the inclusion of additional predictors such as change and exploration of multilevel and recursive models to fully understand the complexities of the relationships involved in WIM. Future research related to methodological issues concern the use of longitudinal or experimental research techniques to identify causation and the generalizability of findings from the sample to the population. These improvements in modelling are further discussed below and another future research direction regarding equivalence testing is raised.

The constructs identified in this thesis occur at different levels of measurement; for example some refer to the organisation (the NZDF), or the participant's service, others refer to the workgroup, or the individual. Constructs at one level can affect constructs at another level. Taking a multilevel modelling approach to the relationships among constructs is something that would be interesting to explore, and the data lend themselves to this sort of modelling. Multilevel modelling would allow for a more sophisticated understanding of employee attitudes and their relationship to WIM.

Other WIM researchers have raised the issue of the recursive nature of WIM in organisations. For example, Einarsen, Hoel, Zapf and Cooper (2003) noted that the effects of WIM on the organisation (such as increased turnover or costs associated with litigation) may in turn influence the action taken by the organisation in response to WIM, and Bentley et al, (2009) propose that stress can be both a predictor and an outcome of WIM. A model that represents and tests the recursive nature of relationships such as culture, climate, leadership behaviour, stress, organisational commitment, and WIM would contribute empirically to the debate around how these constructs influence each other.

The data used in this thesis comes from a diverse sample (particularly military and civilian personnel, and deployed and non-deployed personnel). It may be that different structures of constructs and relationships between constructs hold for different groups. The different possible structures are something that could be investigated further in future research. It would be particularly theoretically interesting and practically useful to contrast models of WIM across 
military and civilian employees to investigate the different contribution of predictors and outcomes. Additionally, some variables were removed from the current analysis due to concerns about missing data. These variables should be included in any modelling of groups which they specifically relate to, and may produce more comprehensive models.

The structure of WIM has long been debated, and existing research has identified a number of important antecedents and consequences of WIM. However, as a result of this thesis we now know that the WIM construct has a complex structure across observed and experienced and across multiple types of WIM. We also know what demographic predictors are not as important as workplace predictors of WIM and that the outcomes of WIM include leaving intentions, mediated by stress, job satisfaction and organisational commitment. The final contribution of this thesis; the identification of constructs measured by the attitude survey, is particularly useful to the NZDF. This final contribution meets a central requirement of good collaborative research, which is that both academia and organisations benefit in some way from the research. 


\section{REFERENCES}

Allen, N. J., \& Meyer, J.P. (1990). The measurement and antecedents of affective, continuance and normative commitment to the organization. Journal of Occupational Psychology, 63, 1-18.

Archer, D. (1999). Exploring "bullying" culture in the para-military organisation. International Journal of Manpower, 20 (1/2), 94-105.

Anderson, N., Herriot, P., \& Hodgkinson, G. P. (2001). The practitioner-researcher divide in industrial, work and organizational (IWO) psychology: where are we now, and where do we go from here? Journal of Occupational and Organizational Psychology, 74, 391-411.

Anderson, C.A., Anderson, K. B., \& Deuser, W. E. (1996). Examining an affective aggression framework: Weapon and temperature effects on aggressive thoughts, affects and attitudes. Personality and Social Psychology Bulletin, 22, 366-376.

Andersson, L. M. \& Pearson, C. M. (1999). Tit for tat? The spiralling effect of incivility in the workplace. Academy of Management Review, 24 (3), 452-471.

Barling, J., Dupré, K.E., \& Kelloway, E.K., (2009). Predicting workplace aggression and violence. Annual Review of Psychology, 6, 671-92.

Bartunek, J. M. (2007) Academic-practitioner collaboration need not require joint or relevant research: Toward a relational scholarship of integration. Academy of Management Journal, 50(6), 1323-1333.

Baruch, Y., \& Holtom, B. C. (2008). Survey response rate levels and trends in organizational research. Human Relations, 61(8), 1139-1160.

Beehr, T. A., Glazer, S., Fischer, R., Linton, L. L., \& Hansen, C. P. (2009). Antecedents for achievement of alignment in organizations. Journal of Occupational and Organisational Psychology, 82(1), $1-20$.

Bentler, P.M. (1990). Comparative fit indexes in structural models. Psychological Bulletin, 107, 238246. 
Bentler, P.M., \& Bonett, D.G. (1980). Significance tests and goodness of fit in the analysis of covariance structures. Psychological Bulletin, 88, 588-606.

Bentley, T., Catley, B., Cooper-Thomas, H., Gardner, D., O’Driscoll, M., \& Trenberth, L. (2009). Understanding Stress and Bullying in New Zealand Workplaces: Final report to OH\&S Steering Committee. The Department of Labour and Health Research Council of New Zealand: New Zealand.

Bergman, M. E., Langhout, R. D., Palmieri, P. A., Cortina, L. M., \& Fitzgerald, L. F. (2009). The (un)reasonableness of reporting: antecedents and consequences of reporting sexual harassment. Journal of Applied Psychology, 87(2), 230-242.

Bildt, C. (2005). Sexual harassment: relation to other forms of discrimination and to health among women and men. Work, 24(3), 251-259.

Bjorkqvist, K., Osterman, K., \& Hjelt-Back, M. (1994). Aggression among university employees. Aggressive behaviour, 20, 173-184.

Black, R. A. (1990). Facts, creativity, teamwork and rules: Understanding leadership styles. Industrial Management, 32(5), 17-21.

Bowling, N A., \& Beehr, T. A. (2006). Workplace harassment from the victim's perspective: A theoretical model and meta-analysis. Journal of Applied Psychology, 91(5), 998-1012.

Brodsky, C.M. (1976). The harassed worker. Toronto: Lexington Books.

Browne, M.W., \& Cudeck, R. (1993). Alternative ways of assessing model fit. In K.A. Bollen \& J.S. Long (Eds.), Testing structural equation models. Newbury Park, CA: Sage.

Cammann, C., Fichman, M., Jenkins, D., \& Klesh, J. R. (1983). The Michigan Organizational Assessment Questionnaire. In S. E. Seashore, E. E. Lawler, \& C. Cammann, (Eds.), Assessing organizational change: A guide to methods, measures, and practices. New York: Wiley. 
Cohen, S., Kamarck, T., \& Mermelstein, R.., (1983). A global measure of perceived stress. Journal of Health and Social Behavior, 24, 385-396.

Cooper, C. L., \& Mashall, J. (1976). Occupational sources of stress: A review of the literature relating to coronary heart disease and mental ill-health. Journal of Occupational Psychology, 49, 11 28.

Cooper-Thomas, H., Catley, B., Bentley, T., Gardner, D., O’Driscoll, M., \& Trenberth, L. (2011). What are the implications of being an observer of workplace bullying? Paper presented at the Auckland Industrial and Organizational Special Interest Group, Human Resources Institute of New Zealand, 12 July 2011, Auckland, NZ.

Cortina L. M., \& Magley V.J. (2003). Raising voice, risking retaliation: Events following interpersonal mistreatment in the workplace. Journal of Occupational Health Psychology, 8(4), 247265.

Cortina, L. M., Magley, V. J., Williams, J. H., \& Langhout, R. D. (2001). Incivility in the workplace: Incidence and impact. Journal of Occupational Health Psychology, 6(1), 64-80.

Crampton, S. M., \& Wagner, J. A. (1994). Percept-percept inflation in microorganizational research: An investigation of prevalence and effect. Journal of Applied Psychology, 79, 67-76.

Cunningham, E. (2008). A practical guide to structural equation modelling using AMOS. Melbourne, Australia: Statsline.

Curry, J. P., Wakefield, D. S., Price, J. L., \& Mueller, C. W. (1986). On the causal ordering of job satisfaction and organizational commitment. Academy of Management Journal, 29(4), 847-856.

Department of Labour. (2003). A Guide to the Health and Safety in Employment Act 1992: Including the Health and Safety in Employment Amendment Act 2002. Wellington, NZ: Occupational Safety and Health Service of the Department of Labour.

Dick, G., \& Rayner, C. (2004). Exploring the workplace bullying construct: An evidence-based approach. University of Kent Canterbury Business School Working Paper Series, 51. 
Dion, M. J. (2006). The impact of work place incivility and occupational stress on the job satisfaction and turnover intention of acute care nurses. Ph.D. Dissertation, University of Connecticut, United StatesConnecticut.

Donovan, M. A., \& Drasgow, F. (1999). Do men's and women's experiences of sexual harassment differ? An examination of the differential test functioning of the sexual experiences questionnaire. Military Psychology, 11(3), 265-282.

Einarsen, S., (1999). The nature and causes of bullying at work. International Journal of Manpower, 20(1/2), 16-27.

Einarsen, S. (2000). Harassment and bullying at work: A review of the Scandinavian approach. Aggression and Violent Behavior, 5(4), 379-401.

Einarsen, S., \& Mikkelsen, E. G. (2003). Individual effects of exposure to bullying at work. In S. Einarsen, H. Hoel, D. Zapf, \& C. L. Copper, (Eds.), Bullying and emotional abuse in the workplace: International perspectives in research and practice. London: Taylor and Francis.

Einarsen, S., \& Skogstad, A. (1996). Bullying at work: Epidemiological findings in public and private organisations. European Journal of Work and Organizational Psychology, 5, 2, 185-201.

Einarsen, S., Raknes, B. I., \& Matthiesen, S. B. (1994). Bullying and harassment at work and their relationships to work environment quality: An exploratory study. European Work and Organizational Psychologist, 4(4), 381-401.

Einarsen, S., Hoel, H., Zapf, D., \& Copper, C. L. (2003). The concept of bullying at work: the European Tradition. In S. Einarsen, H. Hoel, D. Zapf, \& C. L. Copper, (Eds.), Bullying and emotional abuse in the workplace: International perspectives in research and practice. London: Taylor and Francis.

Einarsen, S., Hoel, H., \& Notelaers, G. (2009). Measuring bullying and harassment at work: Validity, factor structure, and psychometric properties of the Negative Acts Questionnaire Revised. Work \& Stress, 23(1), 24-44. 
Ensher, E. A., Grant-Vallone, E. J., \& Donaldson, S. I. (2001). Effects of perceived discrimination on job satisfaction, organizational commitment, organizational citizenship behaviour and grievances. Human Resources Development Quarterly, 12(1), 53-72.

Field, A. (2005) Discovering statistics using SPSS (2 $2^{\text {nd }}$ ed.). London: Sage Publications.

Fischer, R., \& Mansell, A. (2009). Commitment across cultures: A meta-analytical approach. Journal of International Business Studies, 40(8), 1339-1358.

Fitzgerald, L., Drasgow, F., Magley, V., \& Waldo, C. (1999). Measuring sexual harassment in the military: The sexual experiences questionnaire (SEQ-DoD). Military Psychology, 11(3), 243-263.

Fitzgerald, L., Drasgow, F., Hulin, C. L., Gelfand, M. J., \& Magley, V. (1997). Antecedents and consequences of sexual harassment in organisations: a test of an integrated model. Journal of Applied Psychology, 82(4).

Fitzgerald, L. F., Drasgow, F., \& Magley, V. (1999). Sexual harassment in the armed forces: a test of an integrated model. Military Psychology, 11(3), 243-263.

Fitzgerald, L. F., Hulin, C., \& Drasgow, F. (1995). The antecedents and consequences of sexual harassment in organizations: An integrated process model. In S. Sauter \& G. Keita (Eds.). Job stress 2000: Emergent issues. Washington, DC: American Psychological Association.

Gerbing, D. W., \& Hamilton, J. G. (1996). Viability of exploratory factor analysis as a precursor to confirmatory factor analysis. Structural Equation Modelling, 3, 62-72.

Giga, S. I., Cooper, C. L., \& Faragher, B. (2003). The development of a framework for a comprehensive approach to stress management interventions at work. International Journal of Stress Management, 10(4), 280-296.

Gillespie, M. A., Denison, D. R., Haaland, S., Smerek, R., \& Neale, W.S. (2008). Linking organizational culture and customer satisfaction: Results from two companies in different industries. European Journal of Work and Organizational Psychology, 17(1) 112-132.

Gilson, L. L., Mathieu, J. E., Shalley, C. E., \& Ruddy, T. M. (2005). Creativity and standardization: Complementary or conflicting drivers of team effectiveness. Academy of Management Journal, 48, 521-531. 
Glomb, T. M., \& Liao, H. (2003). Interpersonal aggression in work groups: Social influence, reciprocal, and individual effects. Academy of Management Journal, 46, 486-496.

Griffin, B. (2009). From bad manners to bullying: Construct clarification. Paper presented at the $24^{\text {th }}$ Annual Conference of the Society for Industrial and Organizational Psychology, New Orleans, LA.

Groysberg, B., Hill, A., \& Johnson, T. (2010). Which of these people is your future CEO?: The difference way military experience prepares managers for leadership. Harvard Business Review, Nov 2010, 81-85.

Gutek, B. A. (1995). How subjective is sexual harassment? An examination of rater effects. Basic and Applied Social Psychology, 17(4), 447-467.

Hanisch, K. A., \& Hulin, C. L. (1990). Job attitudes and organizational withdrawal: An examination of retirement and other voluntary withdrawal behaviors. Journal of V ocational Bebavior Volume 37( 1), 60-78.

Hershcovis, M. S., Turner, N., Barling, J., Arnold, K. A.., Dupré, K. E., Inness, M., LeBlanc, M. M., \& Sivanathan, N. (2007). Predicting workplace aggression: A meta-analysis. Journal of Applied Psychology, 92(1), 228-238.

Hinkin, T., Holtom, B. C., \& Klag, M. (2007). Collaborative research: Developing mutually beneficial relationships between researchers and organizations. Organizational Dynamics, 36(1), 105-118.

Hoel, H., Einarsen, S., \& Cooper, C. L. (2003). Organisational effects of bullying. In S. Einarsen, H. Hoel, D. Zapf, \& C. L. Copper, (Eds.), Bullying and emotional abuse in the workplace: International perspectives in research and practice. London: Taylor and Francis.

Hoel, H., \& Salin, D. (2003). Organisational antecendents of workplace bullying. In S. Einarsen, H. Hoel, D. Zapf, \& C. L. Copper, (Eds.), Bullying and emotional abuse in the workplace: International perspectives in research and practice. London: Taylor and Francis.

Hoel, H., Glaso, L., Hetland, J., Cooper, C. L., \& Einarsen, S. (2010). Leadership styles as predictors of self-reported and observed workplace bullying. British Journal of Management, 21, 453-468. 
Holt, D. T., Rehg, M. T., Lin, J. H.S., \& Miller, J. (2007). An application of the unfolding model to explain turnover in a sample of military officers. Human Resource Management, 46 (1), 35-49.

Hu, L., \& Bentler, P. M. (1998). Fit indices in covariance structure modeling: Sensitivity to underparameterized model misspecification. Psychological Methods, 3, 424-453.

Hulin, C. L., Fitzgerald, L. F., \& Drasgow, F.(1996). Organizational influences on sexual harassment. Sexual harassment in the workplace: Perspectives, frontiers and response strategies, In M. S. Stockdale (Ed), Sexual harassment in the workplace: Perspectives, frontiers and response strategies, Women and work: $A$ research and policy series, 5, 127-150. Thousand Oaks, CA, USA: Sage Publications.

Hurley, A. E., Scandura, T. A., Schriesheim, C. A., Brannick, M. T., Seers, A., Vandenberg, R. J., \& Williams, L. J. (1997). Exploratory and confirmatory factor analysis: guidelines, issues, and alternatives. Journal of Organizational Behavior, 18, 667-683.

Hutchinson, M., Wilkes, L., Jackson, D., \& Vickers, M. H. (2010). Integrating individual, work group and organisational factors: testing a multidimensional model of bullying in the nursing workplace. Journal of Nursing Management, 18( 2), 173-181.

Ilies, R., Hauserman, N., Schwochau, S., \& Stibal, J. (2003). Reported incidence rates of workrelated sexual harassment in the United States: Using meta-analysis to explain reported rate disparities. Personnel Psychology, 56, 607-631.

Jöreskog, K. G. (1993). Testing structural equation models. In K. A. Bollen \& J. S. Long (Eds.), Testing structural equation models. Newbury Park: Sage.

Kalliath, T., Brough, P., O’Driscoll, M., Manimala, M. J., \& Siu, O. (2010). Organisational Behaviour: A Psychological Perspective for the Asia-Pacific. Australia: McGraw-Hill Australia Pty Ltd

Kearney, S. (2009). Stigma, Well-Being and HDB Survey. Paper presented at the NZ Psychological Society Conference, Palmerston North, NZ. 
Keashly, L., \& Jagatic, K. (2000). The nature, extent and impact of emotional abuse in the workplace: Results of a statewide survey. Paper presented at the Academy of Management Conference, Toronto, Canada.

Keashly, L., \& Jagatic, K. (2003) US Perspectives on Workplace Bullying. In S. Einarsen, H. Hoel, D. Zapf, \& C. L. Copper, (Eds.), Bullying and emotional abuse in the workplace: International perspectives in research and practice. London: Taylor and Francis.

Kessler, R. C., Mickleson, K. D., \& Williams, D. R. (1999). The prevalence, distribution and mental health correlates of perceived discrimination in the United Sates. Journal of Health and Social Behaviour, 40, 208-230.

Lapierre, L M., Spector, P E., \& Leck, J D. (2005). Sexual versus nonsexual workplace aggression and victims' overall job satisfaction: A meta-analysis. Journal of Occupational Health Psychology, 10(2), 155-169.

Larson, S. A., Lakin, K. C., \& Bruininks, R. H. (1998). Staff recruitment and retention: Study results and intervention strategies. Washington, American Association of Mental Retardation.

Law, R., Dollard, M. F., Tuckey, M. R., \& Dormann, C. (2011). Psychosocial safety climate as a lead indicator of workplace bullying and harassment, job resources, psychological health and employee engagement. Accident Analysis and Prevention, 43, 1782-1793.

Lengnick-Hall, M. (1995). Sexual harassment research: A methodological critique. Personnel Psychology, 48, 841-864.

Lewis, D., \& Rayner, C. (2003). Bullying and human resource management: A wolf in sheep's clothing? In S. Einarsen, H. Hoel, D. Zapf, \& C. L. Copper, (Eds.), Bullying and emotional abuse in the workplace: International perspectives in research and practice. London: Taylor and Francis.

Leymann, H. (1996). The content and development of mobbing at work. European Journal of Work and Organizational Psychology, 5(2) 165-184.

Liddell Hart, B. H. (1967). Strategy . London: Faber 
Lim, S., \& Cortina, L. M. (2005). Interpersonal mistreatment in the workplace: the interface and impact of general incivility and sexual harassment. Journal of Applied Psychology, 90(3), 483-896.

Loh, J., Restubog, S. L., \& Zagenczyk, T. J. (2010). Consequences of workplace bullying in employee identification and satisfaction among Australians and Singaporeans. Journal of CrossCultural Psychology, 41(2), 236-252.

McCall, R. B., \& Appelbaum, M. I. (1991). Some Issues of Conducting Secondary Analyses. Developmental Psychology, 27 (6), 911-917.

Notelaers, G., De Witte, H., \& Einarsen, S. (2010). A job characteristics approach to explain workplace bullying. European Journal of Work and Organizational Psychology, 19(4), 487-504.

Mageroy, N., Lau, B., Riise, T., \& Moen, B. E. (2009). Association of psychosocial factors and bullying at individual and department levels among naval military personnel. Journal of Psychosomatic Research, 66, 343-351.

Magley, V. J., Waldo, C. R., Drasgow, F., \& Fitzgerald, L. F. (1999). The impact of sexual harassment on military personnel: is it the same for men and women? Military Psychology, 11(3), 283-302.

Manning, F. (1994). Morale and Cohesion in Military Psychiatry. In F. D. Jones (Ed.) Military Psychiatry: Preparing in Peace for $W$ ar. USA: Department of the Army, Office of the Surgeon General, Borden Institute

Marsh, H. W., Hau, K. T., \& Wen, Z. L. (2004). In search of golden rules: Comment on hypothesis testing approaches to setting cutoff values for fit indexes and dangers in overgeneralising Hu \& Bentler (1999) findings. Structural Equation Modeling, 11, 320-341.

Mathisen, G. E., Einarsen, S., \& Mykletun, R. (2008). The occurrences and correlates of bullying and harassment in the restaurant sector. Scandinavian Journal of Psychology, 49, 59-68.

McCarthy, P., \& Mayhew, C. (2004). Safeguarding the Organisation against Violence and Bullying: An International Perspective. New York: Palgrave MacMillan.

Munson, L. J., Miner, A. G., \& Hulin, C. (2001). Labelling sexual harassment in the military: An extension and replication. Journal of Applied Psychology, 86(2), 293-303. 
Monks, C. P., Smith, P. K., Naylor, P., Barter, C., Ireland J. L., \& Coyne, I. (2009). Bullying in different contexts: Commonalities, differences and the role of theory. Aggression and Violent Behavior, 14 (2), 146-156.

Neal, A., \& Griffin, M. A. (2006). A study of the lagged relationships among safety climate, safety motivation, safety behaviour and accidents at the individual and group levels. Journal of Applied Psychology, 91 (4), 946-953.

Neuman, J., \& Baron, R. (1998). Workplace violence and workplace aggression: Evidence concerning specific forms, potential causes, and preferred targets. Journal of Management 24(3), 391-419.

Nye, C. D., Brummel, B. J., \& Drasgow, F. (2009). Differentiating gender discrimination and sexist behaviour: an examination of antecedents and outcomes. Military Psychology, 21(3), 299314.

O'Connor, B. (2000). SPSS and SAS programs for determining the number of components using parallel analysis and Velicer's MAP test. Behavior Research Methods, Instruments and Computers, 32(3), 396-402.

Ostvik, K., \& Rudmin, F (2001). Bullying and hazing among Norwegian Army soldiers: two studies of prevalence, context and cognition. Military Psychology, 13(1), 17-39.

Parker, D. E., \& DeCotiis, T. A. (1983). Organizational Determinants of Job Stress. Organizational Behavior and Human Performance, 32, 160-177.

Pavalko, E, K., Mossakowski, K. N., \& Hamilton, V. J. (2003). Does perceived discrimination affect health? Longitudinal relationships between work discrimination and women's physical and emotional health. Journal of Health and Social Behaviour, 43, 18-33.

Pearce, C. L., \& Sims Jr., H. P. (2002). Vertical versus shared leadership as predictors of the effectiveness of change management teams: An examination of aversive, directive, transactional, transformational, and empowering leader behaviors. Group Dynamics: Theory, Research, and Practice, 6 (2), 172-197. 
Pryor, J. B., \& Fitzgerald, L. F. (2003). Sexual harassment research in the United States. In S. Einarsen, H. Hoel, D. Zapf, \& C. L. Copper, (Eds.), Bullying and emotional abuse in the workplace: International perspectives in research and practice. London: Taylor and Francis.

Raver, J. L., \& Nishii, L. N. (2010). Once, twice or three times as harmful? Ethnic harassment, gender harassment and generalised workplace harassment. Journal of Applied Psychology, 95 (2), 236-254.

Rayner, C. (1999). Workplace bullying. PhD thesis, UMIST.

Rayner, C., \& Hoel, H. (1997). A summary review of literature relating to workplace bullying. Journal of Community and Applied Social Psychology, 7, 181-191.

Rayton, B. A. (2006). Examining the interconnection of job satisfaction and organizational commitment: An application of the bivariate probit model. The International Journal of Human Resource Management, 17(1), 139-154.

Saks, A. M. (2006). Antecedents and consequences of employee engagement. Journal of Managerial Psychology, 21 (7), 600-619.

Salin, D. (2001). Prevalence and forms of bullying among business professionals: A comparison of two different strategies for measuring bullying. European Journal of Work and Organizational Psychology, 10 (4), 425-441.

Salin, D. (2003). Ways of explaining workplace bullying: A review of enabling, motivating and precipitating structures and processes in the work environment. Human Relations, 56(10), 12131231.

Schein, E. H. (2004). Organizational Culture and Leadership. California: John Wiley and Sons.

Semler, S. W. (1997). Systemic agreement: A theory of organizational alignment. Human Resource Development Quarterly, 8 (1), 23-40.

Gilboa, S., Shirom, A., Fried, Y., \& Cooper, C. (2008). A meta-analysis of work demand stressors and job performance: Examining main and moderating effects. Personnel Psychology, 61, 227-271. 
Spector, P. E., \& Jex, S. M. (1998). Development of four self-report measures of job stressors and strain: Interpersonal Conflict at Work Scale, Organisational Constraints Scale, Quantitative Workload Inventory, and Physical Symptoms Inventory. Journal of Occupational Health Psychology, 3, 356-366.

Scarville, J., Button, S., Edwards, J., Lancaster, A., \& Elig, T. (1999). Armed forces equal opportunity survey. (ADA-366037). Arlington, VA: Defense Manpower Data Center.

Sims, C. S., Drasgow, F., \& Fitzgerald, L. F. (2005). The effects of sexual harassment on turnover in the military: time-dependent modelling. Journal of Applied Psychology, 90 (6).

Stanton, J. M., Balzer, W. K., Smith, P. C., Parra, L. F., \& Ironson, G. (2001). A general measure of work stress: the stress in general scale. Educational and Psychological Measurement, 61, 866.

Stevens, J. P. (1992) Applied multivariate statistics for the social sciences (2d ed.). Hillsdale, NJ: Erlbaum.

Tabachnick, B. G., \& Fidell, L. S. (2007). Using Multivariate Statistics (5th ed.). Boston, MA: Pearson.

Tanaka, J. S. (1993). Multifacted conceptions of fit in structural equation models. In K.A. Bollen \& J.S. Long (Eds.), Testing structural equation models. Newbury Park, CA: Sage.

Taylor, T. (2003). Jointery: Military Integration. In T. McConville \& R. Holmes (Eds.), Defence Management in Uncertain Times. London: Frank Cass Publishers.

Tepper, B. J. (2000). Consequences of Abusive Supervision. The Academy of Management Journal, 43, (2), 178-190.

Truhon, S. A. (2008). Equal opportunity climate in the United States military: Are differences in the eye of the beholder? European Journal of Work and Organizational Psychology, 17(1), 153-169.

Turok, I., Kearns, A., Fitch, D., Flint, J., McKenzie, C., \& Abbotts, J. (2006). State of the English Cities: Social Cohesion. West Yorkshire, UK: Department for Communities and Local Government.

Van Creveld, M. (1989). Technology and War. London: Collier Macmillan. 
Vartia, M.A. L. (2001). Consequences of workplace bullying with respect to the well-being of its targets and the observers of bullying. Scandinavian Journal of Work and Environmental Health, 27(1), 63-69.

Walsh, B. M., Matthews, R. A., Tuller, M. D., Parks, K. M. \& McDonald, D. P. (2010). A multilevel model of the effects of equal opportunity climate on job satisfaction in the military. Journal of Occupational Health Psychology, 15(2), 191-207.

Williams, E. S., Konrad, T. R., Scheckler, W. E., Pathman, D. E., Linzer, M., McMurray, J. E., Gerrity M.,\& Schwartz M. (2001).Understanding Physicians' Intentions to Withdraw from Practice: The Role of Job Satisfaction, Job Stress, Mental and Physical Health. Advances in Health Care Management, 2, 243-262.

Williams, J. H., Fitzgerald, L. F., \& Drasgow, F. (1999). The effects of organizational practices on sexual harassment and individual outcomes in the military. Military Psychology, 11(3), 303-328.

Williams, K. (1997). Social ostracism. In R. M. Kowalski (Ed.), Aversive interpersonal behaviours. New York: Plenum Press.

Willness, C. R., Steel, P., \& Lee, K. (2007). A meta-analysis of the antecedents and consequences of workplace sexual harassment. Personnel Psychology, 60, 127-162.

Winsborough, D., Morris, M., \& Hughes, M. (2008). Preventative defence against attrition: Engaging 'on the fence' employees. Selection \& Development Review, 24(2), 3-7.

Wither, J. K. (2004). Battling bullying in the British Army 1987 - 2004. The Journal of Power Institutions in Post-Soviet Societies, 1 Retrieved 2 October 2011, http://pipss.revues.org/index46.html.

Yildirim, D. (2009). Bullying among nurses and its effects. International Nursing Review, 56, 504511.

Zapf, D., Einarsen, S., Hoel, H., \& Varita, M. (2003). Findings on bullying in the workplace. In S. Einarsen, H. Hoel, D. Zapf, \& C. L. Copper, (Eds.), Bullying and emotional abuse in the workplace: International perspectives in research and practice. London: Taylor and Francis. 
Zapf, D., \& Einarsen, S. (2003). Individual antecedents of bullying, In S. Einarsen, H. Hoel, D.

Zapf, \& C. L. Copper, (Eds.), Bullying and emotional abuse in the workplace: International perspectives in research and practice. London: Taylor and Francis. 


\title{
APPENDIX A: NZDF Ongoing Attitude Survey
}

\author{
NEW ZEALAND DEFENCE FORCE \\ ATTITUDE SURVEY
}

You have been selected as part of a random sample for this month's administration of the NZDF Ongoing Attitude Survey, conducted by HQ NZDF Personnel Branch. The survey asks you about your satisfaction with matters such as supervision, work conditions, career management, change and equity. This survey is sent out to all NZDF civilian and military personnel on an ongoing basis.

The information you provide will be used for two purposes:

- To assist in decision-making regarding current management practice and policy decisions.

- To monitor trends over time.

From time to time the survey information may be used to inform NZDF supported research conducted by internal and external researchers.

This survey is administered in accordance with the Privacy Act (1993) and guidelines for research practice outlined in DFO 21/2002. The information collected from respondents will be treated strictly as "in-confidence". The information you provide will never be used to evaluate your performance, and legislation prohibits us from publishing information that identifies you.

What happens to your information?

- Completed surveys are sent directly to The Directorate of Psychology, where responses are entered into a central database. Once surveys have been entered into the database, the original surveys are destroyed.

- Reports focus on percentages and relationships. Reports never specify individual responses.

- Service Psychologists receive copies of the database for their own analysis. These databases do not contain specific information to identify you.

- Individual information from the surveys will not be given out to anyone for any reason.

In the survey you are asked to provide your Service Number. This will allow us to link your survey responses with a range of personal information (trade, service, age, length of service, etc) from the personnel information system (ATLAS) so that you do not have to provide this information yourself. This will allow us to determine whether there are differences in opinions or levels of satisfaction across groups such as rank and trade.

I acknowledge that taking the time to complete this survey competes with other demands in your work place. I believe there is significant benefit in the conduct of this survey and I strongly encourage you to complete it. Your opinions are important to shaping effective future human resource policy and practice. I give you an assurance that the comments and information generated in response to survey questions will be listened to.

J. MATEPARAE

Lieutenant General

Chief of Defence Force

It is very important that you take the time to complete this survey honestly and thoughtfully. Please read the instructions overleaf. 


\section{Instructions}

If you have access to the NZDF Intranet you may choose to fill this questionnaire in online at the following address: http://ilp/FrameTool.asp?Target=http://dcsbhost/survey/O AtS.htm

1. There are no right or wrong answers. It is very important that your answers accurately reflect your own experiences and opinions.

2. The questions relate to three areas within the NZDF. Please think about the area specified when answering each question:
a. Your immediate workplace
b. Your Service
c. The NZDF as a whole - remember that your Service is part of that organisation.

3. When you are required to make a choice between answers, please completely darken the circle that corresponds with your response.
Like this:
$\bigcirc \bigcirc \bigcirc \bigcirc$ AND
$\stackrel{1}{0} \stackrel{2}{0}^{3} \stackrel{4}{0}^{\circ} \stackrel{6}{0}^{\circ} 0^{7}$

If you make an error, put a cross through it like this

and then darken the circle that corresponds to your correct response.

\section{There are questions on both sides of the pages.}

\section{Do not fold or staple the questionnaire.}

6. When you have finished answering the questionnaire, return it in the self-addressed envelope that you received with the questionnaire.

\section{Your participation in this piece of research is greatly appreciated.}

We hope you take this chance to be heard.

Want to know what happens with your results?

You can view the results of the survey and the outcomes generated on the Personnel Branch web site. 
Please enter your service number

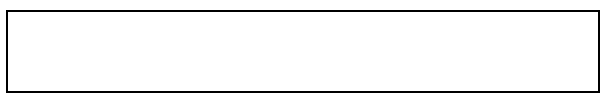

Please enter the current date

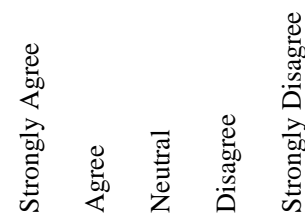

1. Being in the NZDF gives me a sense of belonging to one big family.

$\begin{array}{lllll}0 & 0 & 0 & 0 & 0\end{array}$

2. I care about the future of the NZDF.

0

3. I am willing to put in effort beyond that normally expected in order to help the NZDF be successful.

4. The NZDF is the best possible organisation to work for.

$\begin{array}{llllllll}0 & 0 & 0 & 0 & 0\end{array}$

5. I would rather work for a different organisation as long as the type of work and pay was similar.

$\begin{array}{lllll}0 & 0 & 0 & 0 & 0\end{array}$

6. I speak highly of my Service/the NZDF to my friends.

$\begin{array}{lllllllll}0 & 0 & 0 & 0 & 0\end{array}$

7. In the NZDF, the more you do the more you are expected to do.

$\begin{array}{lllll}0 & 0 & 0 & 0 & 0\end{array}$

8. I think I am doing something worthwhile for my country by being in the NZDF.

$\begin{array}{lllll}0 & 0 & 0 & 0 & 0\end{array}$

9. I feel there is not much to be gained by staying in the NZDF.

$\begin{array}{lllll}0 & 0 & 0 & 0 & 0\end{array}$

10. Deciding to join the NZDF was a definite mistake on my part.

$\begin{array}{lllll}0 & 0 & 0 & 0 & 0\end{array}$

11. I find that my values and the NZDF's values are very similar.

$\begin{array}{lllll}0 & 0 & 0 & 0 & 0\end{array}$

12. I understand the mission, goals, and objectives of the NZDF.

$\begin{array}{lllll}0 & 0 & 0 & 0 & 0\end{array}$

13. I understand how I contribute to the NZDF's mission.

$\begin{array}{lllll}0 & 0 & 0 & 0 & 0\end{array}$

14. The future prospects of the NZDF are good.

$\begin{array}{lllll}0 & 0 & 0 & 0 & 0\end{array}$

15. My loyalty to the NZDF is matched by its loyalty to me.

$\begin{array}{lllll}0 & 0 & 0 & 0 & 0\end{array}$

16. I feel there is a future for me in the NZDF.

$\begin{array}{lllll}0 & 0 & 0 & 0 & 0\end{array}$

17. I believe in what the NZDF is trying to accomplish.

$\begin{array}{lllll}0 & 0 & 0 & 0 & 0\end{array}$

$\begin{array}{lllll}0 & 0 & 0 & 0 & 0\end{array}$

18. I am proud to tell others I am a member of my Service/the NZDF.

$\begin{array}{lllll}0 & 0 & 0 & 0 & 0\end{array}$

Which statement about Organisational Commitment do you consider is the most important to you?

$\begin{array}{ccccccccccccccccccc}\text { None } & 1 & 2 & 3 & 4 & 5 & 6 & 7 & 8 & 9 & 10 & 11 & 12 & 13 & 14 & 15 & 16 & 17 & 18 \\ \mathrm{O} & \mathrm{O} & \mathrm{O} & \mathrm{O} & \mathrm{O} & \mathrm{O} & \mathrm{O} & \mathrm{O} & \mathrm{O} & \mathrm{O} & \mathrm{O} & \mathrm{O} & \mathrm{O} & \mathrm{O} & \mathrm{O} & \mathrm{O} & \mathrm{O} & \mathrm{O} & \mathrm{O}\end{array}$




\section{Section 2: Job Satisfaction}

1. I feel I have more to do than I can handle.

2. My current working hours are too long.

$\begin{array}{lllll}0 & 0 & 0 & 0 & 0\end{array}$

3. I am paid appropriately for the job I perform.

$\begin{array}{lllll}0 & 0 & 0 & 0 & 0\end{array}$

4. My pay is comparable to what I would get outside the organisation.

$\begin{array}{lllll}0 & 0 & 0 & 0 & 0\end{array}$

5. The pay and benefits I receive are fair for the work I do.

$\begin{array}{lllll}0 & 0 & 0 & 0 & 0\end{array}$

6. Much of what I do in the NZDF is interesting.

$\begin{array}{lllll}0 & 0 & 0 & 0 & 0\end{array}$

7. I like the work in my present position.

$\begin{array}{lllll}0 & 0 & 0 & 0 & 0\end{array}$

8. My work makes an important contribution to the NZDF.

$\begin{array}{lllll}0 & 0 & 0 & 0 & 0\end{array}$

9. I have a great deal of pride in my work.

$\begin{array}{lllll}0 & 0 & 0 & 0 & 0\end{array}$

10. I am often being asked for my ideas about how we could do things better at work.

$\begin{array}{lllll}0 & 0 & 0 & 0 & 0\end{array}$

11. I enjoy working with the people in my workplace.

$\begin{array}{lllll}0 & 0 & 0 & 0 & 0\end{array}$

12. In my workplace we are continually looking at ways to do things better.

$\begin{array}{lllll}0 & 0 & 0 & 0 & 0\end{array}$

13. The quality of the work done in my workplace is very important to me.

$\begin{array}{lllll}0 & 0 & 0 & 0 & 0\end{array}$

14. Most of the time my job is repetitive and boring.

$\begin{array}{lllll}0 & 0 & 0 & 0 & 0\end{array}$

15. I do my current job well.

$\begin{array}{lllll}0 & 0 & 0 & 0 & 0\end{array}$

16. I have the freedom and flexibility I need to do my job effectively.

$\begin{array}{lllll}0 & 0 & 0 & 0 & 0\end{array}$

17. Life in my Service/the NZDF is enjoyable.

$\begin{array}{lllll}0 & 0 & 0 & 0 & 0\end{array}$

18. Overall, I'm satisfied with my job.

$\begin{array}{lllll}0 & 0 & 0 & 0 & 0\end{array}$

19. Overall, I feel my contribution to the NZDF is valued.

$\begin{array}{lllll}0 & 0 & 0 & 0 & 0\end{array}$

20. My job gives me a sense of personal achievement.

$\begin{array}{lllll}0 & 0 & 0 & 0 & 0\end{array}$

21. The work I do makes full use of my knowledge and skills.

$\begin{array}{lllll}0 & 0 & 0 & 0 & 0\end{array}$

22. I get recognition when I do a good job.

$\begin{array}{lllll}0 & 0 & 0 & 0 & 0\end{array}$

$\begin{array}{lllll}0 & 0 & 0 & 0 & 0\end{array}$

23. I have the opportunity to realise my potential in the NZDF.

$\begin{array}{lllll}0 & 0 & 0 & 0 & 0\end{array}$

Which statement about Job Satisfaction do you consider is the most important to you?

$\begin{array}{ccccccccccccc}\text { None } & 1 & 2 & 3 & 4 & 5 & 6 & 7 & 8 & 9 & 10 & 11 & 12 \\ \mathrm{O} & \mathrm{O} & \mathrm{O} & \mathrm{O} & \mathrm{O} & \mathrm{O} & \mathrm{O} & \mathrm{O} & \mathrm{O} & \mathrm{O} & \mathrm{O} & \mathrm{O} & \mathrm{O} \\ & 13 & 14 & 15 & 16 & 17 & 18 & 19 & 20 & 21 & 22 & 23 & \\ & \mathrm{O} & \mathrm{O} & \mathrm{O} & \mathrm{O} & \mathrm{O} & \mathrm{O} & \mathrm{O} & \mathrm{O} & \mathrm{O} & \mathrm{O} & \mathrm{O} & \end{array}$


1. I have confidence in the leadership of the NZDF.

2. The NZDF is a fun place to work.

$\begin{array}{lllll}0 & 0 & 0 & 0 & 0\end{array}$

3. I am satisfied with my physical work environment.

$\begin{array}{lllll}0 & 0 & 0 & 0 & 0\end{array}$

4. I know how my work contributes to the success of the NZDF.

$\begin{array}{lllll}0 & 0 & 0 & 0 & 0\end{array}$

5. I can rely on the support of others in the NZDF.

$\begin{array}{lllll}0 & 0 & 0 & 0 & 0\end{array}$

6. We celebrate success in the NZDF.

$\begin{array}{lllll}0 & 0 & 0 & 0 & 0\end{array}$

7. I feel informed about the NZDF and its activities.

$\begin{array}{lllll}0 & 0 & 0 & 0 & 0\end{array}$

8. The NZDF cares about the well-being of its people

$\begin{array}{lllll}0 & 0 & 0 & 0 & 0\end{array}$

9. People share their knowledge and ideas freely in the NZDF.

$\begin{array}{lllll}0 & 0 & 0 & 0 & 0\end{array}$

10. I feel I am working for a successful organisation.

$\begin{array}{lllll}0 & 0 & 0 & 0 & 0\end{array}$

11. The work of the people in my Service is valued by the New Zealand public.

$\begin{array}{lllll}0 & 0 & 0 & 0 & 0\end{array}$

12. The condition and functionality of the building I work in is acceptable.

$\begin{array}{lllll}0 & 0 & 0 & 0 & 0\end{array}$

13. From my experience, the quality of the living accommodation (Service housing or barracks) is acceptable.

$\begin{array}{lllll}0 & 0 & 0 & 0 & 0\end{array}$

Which statement about Organisational Culture do you consider is the most important to you?

$\begin{array}{cccccccccccccc}\text { None } & 1 & 2 & 3 & 4 & 5 & 6 & 7 & 8 & 9 & 10 & 11 & 12 & 13 \\ \mathrm{O} & \mathrm{O} & \mathrm{O} & \mathrm{O} & \mathrm{O} & \mathrm{O} & \mathrm{O} & \mathrm{O} & \mathrm{O} & \mathrm{O} & \mathrm{O} & \mathrm{O} & \mathrm{O} & \mathrm{O}\end{array}$


1. The NZDF provides a good quality of life compared with other organisations.

2. There is not enough time allocated for the pursuit of fitness activities within the NZDF.

3. My work schedule often conflicts with my personal life.

$\begin{array}{lllll}0 & 0 & 0 & 0 & 0\end{array}$

4. I am able to maintain a balance between my personal and working life.

$\begin{array}{lllll}0 & 0 & 0 & 0 & 0\end{array}$

5. The NZDF provides adequate family support.

$\begin{array}{lllll}0 & 0 & 0 & 0 & 0\end{array}$

6. I know where to get help for personal or family issues.

$\begin{array}{lllll}0 & 0 & 0 & 0 & 0\end{array}$

7. My supervisor is flexible when I have family demands to attend to.

$\begin{array}{lllll}0 & 0 & 0 & 0 & 0\end{array}$

8. I need to spend more time with my family.

$\begin{array}{lllll}0 & 0 & 0 & 0 & 0\end{array}$

9. My family is fully supportive of my employment with the NZDF.

$\begin{array}{lllll}0 & 0 & 0 & 0 & 0\end{array}$

$\begin{array}{lllll}0 & 0 & 0 & 0 & 0\end{array}$

10. I want more location stability.

$\begin{array}{lllll}0 & 0 & 0 & 0 & 0\end{array}$

$\begin{array}{lllll}0 & 0 & 0 & 0 & 0\end{array}$

11. Postings have had a negative impact on my children's education.

$\begin{array}{lllll}0 & 0 & 0 & 0 & 0\end{array}$

12. Postings have had a negative impact on my partner's career.

$\begin{array}{lllll}0 & 0 & 0 & 0 & 0\end{array}$

13. Postings have had a negative impact on my life.

$\begin{array}{lllll}0 & 0 & 0 & 0 & 0\end{array}$

14. Military life places/placed a strain on my relationship.

$\begin{array}{lllll}0 & 0 & 0 & 0 & 0\end{array}$

Which statement about Work/Life Balance do you consider is the most important to you?

$\begin{array}{ccccccccccccccc}\text { None } & 1 & 2 & 3 & 4 & 5 & 6 & 7 & 8 & 9 & 10 & 11 & 12 & 13 & 14 \\ \mathrm{O} & \mathrm{O} & \mathrm{O} & \mathrm{O} & \mathrm{O} & \mathrm{O} & \mathrm{O} & \mathrm{O} & \mathrm{O} & \mathrm{O} & \mathrm{O} & \mathrm{O} & \mathrm{O} & \mathrm{O} & \mathrm{O}\end{array}$

\section{Section 5: Health and Safety}

1. There is too much stress in NZDF related areas of my life.

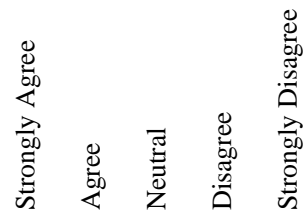

2. There is too much stress in non-NZDF related areas of my life.

$\begin{array}{lllll}0 & 0 & 0 & 0 & 0\end{array}$

3. The level of stress I am experiencing is affecting my personal well-being.

$\begin{array}{lllll}0 & 0 & 0 & 0 & 0\end{array}$

4. The level of stress I am experiencing is affecting my job performance.

$\begin{array}{lllll}0 & 0 & 0 & 0 & 0\end{array}$

5. I cope with the stress of my job.

$\begin{array}{lllll}0 & 0 & 0 & 0 & 0\end{array}$

6. Health and safety is treated as an important issue in my workplace.

$\begin{array}{lllll}0 & 0 & 0 & 0 & 0\end{array}$

7. I have the skills and training to do my job safely.

$\begin{array}{lllll}0 & 0 & 0 & 0 & 0\end{array}$

8. The hazards of my job are controlled so that I am unlikely to be harmed.

$\begin{array}{lllll}0 & 0 & 0 & 0 & 0\end{array}$

9. There are personnel within my workplace who have health and safety responsibilities.

$\begin{array}{lllll}0 & 0 & 0 & 0 & 0\end{array}$

10. I know how to raise any health and safety concerns I may have.

$\begin{array}{lllll}0 & 0 & 0 & 0 & 0\end{array}$

11. I know how to report an accident.

$\begin{array}{lllll}0 & 0 & 0 & 0 & 0\end{array}$

$\begin{array}{lllll}0 & 0 & 0 & 0 & 0\end{array}$

Which statement about Health and Safety do you consider is the most important to you?

$\begin{array}{cccccccccccc}\text { None } & 1 & 2 & 3 & 4 & 5 & 6 & 7 & 8 & 9 & 10 & 11 \\ \mathrm{O} & \mathrm{O} & \mathrm{O} & \mathrm{O} & \mathrm{O} & \mathrm{O} & \mathrm{O} & \mathrm{O} & \mathrm{O} & \mathrm{O} & \mathrm{O} & \mathrm{O}\end{array}$


1. I am well informed about equity and diversity policies in the NZDF.

2. Overall, policies are sensitive to the needs of different groups within the NZDF.

3. We do not focus enough on equity and diversity issues in our workplace.

O 00000

4. The NZDF is an equal opportunity employer.

O 000000

5. I am always treated with courtesy and respect in my workplace.

0000

6. It doesn't matter what gender you are as long as you can handle the job.

Harassment is behaviour that is demeaning to another person's status, leaving the person feeling humiliated, offended or intimidated. Harassment can be repetitive or of a significant nature, is often power based, and is detrimental to the individual.

7. I have observed harassment in the workplace over the past year.

$\begin{array}{lllll}0 & 0 & 0 & 0 & 0\end{array}$

8. I have experienced harassment in the workplace over the past year.

O 00000

Discrimination occurs when a person is treated less favourably than others in the same or similar circumstances based on the specific grounds under the Human Rights Act.

9. I have observed discrimination in the workplace over the past year.

$\begin{array}{lllll}0 & 0 & 0 & 0 & 0\end{array}$

10. I have experienced discrimination in the workplace over the past year.

$\begin{array}{lllll}0 & 0 & 0 & 0 & 0\end{array}$

11. Incidents of discrimination or harassment are managed well in my workplace.

$\begin{array}{lllll}0 & 0 & 0 & 0 & 0\end{array}$

12. I would be confident to approach my superiors if I was being harassed at work.

$\begin{array}{lllll}0 & 0 & 0 & 0 & 0\end{array}$

13. I know what support services are available to me if I were being harassed at work.

$\begin{array}{lllll}0 & 0 & 0 & 0 & 0\end{array}$

Workplace bullying is usually repeated (or a significant single incident), unreasonable behaviour (victimising, humiliating, undermining or threatening) directed toward a person that creates a risk to the mental of physical health and safety of the person.

14. I have observed bullying in the workplace over the past year.

$\begin{array}{lllll}0 & 0 & 0 & 0 & 0\end{array}$

15. I have experienced bullying in the workplace over the past year.

$\begin{array}{lllll}0 & 0 & 0 & 0 & 0\end{array}$

16. The attitude of people in my workplace would make it difficult for people from different ethnic or cultural origins.

$\begin{array}{lllll}0 & 0 & 0 & 0 & 0\end{array}$

17. The attitude of people in my workplace would make it difficult for people who are gay or lesbian.

18. The attitude of people in my workplace would make it difficult for people with disabilities.

$\begin{array}{lllll}0 & 0 & 0 & 0 & 0\end{array}$

$\begin{array}{lllll}0 & 0 & 0 & 0 & 0\end{array}$

Which statement about Equity do you consider is the most important to you?

$\begin{array}{ccccccccccccccccccc}\text { None } & 1 & 2 & 3 & 4 & 5 & 6 & 7 & 8 & 9 & 10 & 11 & 12 & 13 & 14 & 15 & 16 & 17 & 18 \\ \mathrm{O} & \mathrm{O} & \mathrm{O} & \mathrm{O} & \mathrm{O} & \mathrm{O} & \mathrm{O} & \mathrm{O} & \mathrm{O} & \mathrm{O} & \mathrm{O} & \mathrm{O} & \mathrm{O} & \mathrm{O} & \mathrm{O} & \mathrm{O} & \mathrm{O} & \mathrm{O} & \mathrm{O}\end{array}$


1. My immediate supervisor shows good leadership.

2. I trust my immediate supervisor.

3. I respect my immediate supervisor.

$\begin{array}{lllll}0 & 0 & 0 & 0 & 0\end{array}$

4. My immediate supervisor focuses more on my mistakes than on my good work.

$\begin{array}{lllll}0 & 0 & 0 & 0 & 0\end{array}$

5. My immediate supervisor ensures our workplace works well together.

$\begin{array}{lllll}0 & 0 & 0 & 0 & 0\end{array}$

6. My immediate supervisor actively supports a harassment free work environment.

$\begin{array}{lllll}0 & 0 & 0 & 0 & 0\end{array}$

7. My immediate supervisor makes sure I understand his/her role.

$\begin{array}{lllll}0 & 0 & 0 & 0 & 0\end{array}$

8. My immediate supervisor makes clear what is expected of the workplace.

$\begin{array}{lllll}0 & 0 & 0 & 0 & 0\end{array}$

9. My immediate supervisor keeps me informed about relevant matters.

$\begin{array}{lllll}0 & 0 & 0 & 0 & 0\end{array}$

$\begin{array}{lllll}0 & 0 & 0 & 0 & 0\end{array}$

Which statement about Supervision do you consider is the most important to you?

$\begin{array}{cccccccccc}\text { None } & 1 & 2 & 3 & 4 & 5 & 6 & 7 & 8 & 9 \\ \mathrm{O} & \mathrm{O} & \mathrm{O} & \mathrm{O} & \mathrm{O} & \mathrm{O} & \mathrm{O} & \mathrm{O} & \mathrm{O} & \mathrm{O}\end{array}$

\section{Section 8: Senior Leadership}

Note - Senior leadership refers to Capt / Col / Gp Capt / senior managers and above

1. I believe senior leaders have the skills to do the job of leading the NZDF.

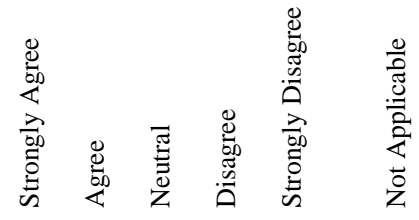

2. Senior leaders are committed to improving the way the NZDF is run.

$\begin{array}{lllll}0 & 0 & 0 & 0 & 0\end{array}$

3. Senior leaders keep people informed about matters that affect their work.

$\begin{array}{lllll}0 & 0 & 0 & 0 & 0\end{array}$

4. Senior leaders' behaviour reflects the values of the NZDF.

$\begin{array}{lllll}0 & 0 & 0 & 0 & 0\end{array}$

5. Communication between senior leaders and Defence personnel is good.

$\begin{array}{lllll}0 & 0 & 0 & 0 & 0\end{array}$

6. Senior leaders create an environment where differences are valued.

$\begin{array}{lllll}0 & 0 & 0 & 0 & 0\end{array}$

7. Senior leaders are receptive to new ideas.

$\begin{array}{lllll}0 & 0 & 0 & 0 & 0\end{array}$

$\begin{array}{lllll}0 & 0 & 0 & 0 & 0\end{array}$

Which statement about Senior Management/Leadership do you consider is the most important to you?

$\begin{array}{cccccccc}\text { None } & 1 & 2 & 3 & 4 & 5 & 6 & 7 \\ \mathrm{O} & \mathrm{O} & \mathrm{O} & \mathrm{O} & \mathrm{O} & \mathrm{O} & \mathrm{O} & \mathrm{O}\end{array}$


1. My immediate supervisor makes clear what tasks I need to perform.

2. My immediate supervisor makes clear what standards are expected in my work.

3. My immediate supervisor provides me with regular feedback on my work.

4. I currently have in place a performance plan that has been developed by my immediate supervisor and me.

5. I agree with the standards against which my performance is formally appraised / reviewed.

6. I am satisfied with the performance appraisal / review system.

7. Overall, my workplace produces high-quality services and outputs.

8. Other members in my workplace have the job relevant knowledge and skills necessary to accomplish unit targets.

9. The feedback and coaching I get helps me to improve my performance

Which statement about Performance Management do you consider is the most important to you?

$\begin{array}{cccccccccc}\text { None } & 1 & 2 & 3 & 4 & 5 & 6 & 7 & 8 & 9 \\ \mathrm{O} & \mathrm{O} & \mathrm{O} & \mathrm{O} & \mathrm{O} & \mathrm{O} & \mathrm{O} & \mathrm{O} & \mathrm{O} & \mathrm{O}\end{array}$

\section{Section 10: Career Development}

1. There are opportunities for advancement / promotion for me within the NZDF.

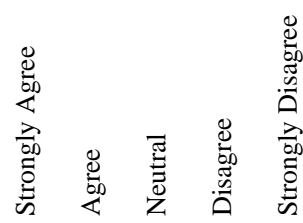

2. My immediate supervisor encourages my personal and professional development.

$\begin{array}{lllll}0 & 0 & 0 & 0 & 0\end{array}$

3. There are career and personal development opportunities for me in the NZDF.
Please respond to statements 4 to 8 only if you are a uniformed Service person.

4. I am satisfied with the lengths of engagement offered.

$\begin{array}{lllllllllll}0 & 0 & 0 & 0 & 0\end{array}$

5. I am satisfied with the way my career is being managed.

$\begin{array}{lllll}0 & 0 & 0 & 0 & 0\end{array}$

6. Promotion processes are fair and equitable.

$\begin{array}{lllll}0 & 0 & 0 & 0 & 0\end{array}$

7. Decisions around promotion are fair and equitable.

8. Information on my career management has been adequate.

Which statement about Career Development do you consider is the most important to you?

$\begin{array}{ccccccccc}\text { None } & 1 & 2 & 3 & 4 & 5 & 6 & 7 & 8 \\ \mathrm{O} & \mathrm{O} & \mathrm{O} & \mathrm{O} & \mathrm{O} & \mathrm{O} & \mathrm{O} & \mathrm{O} & \mathrm{O}\end{array}$




\section{Section 11: Training and Resources}

1. This organisation ensures that I am adequately trained for the work I do.

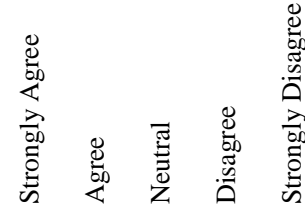

2. Training opportunities are allocated fairly.

$\begin{array}{llllll}0 & 0 & 0 & 0 & 0\end{array}$

3. We have insufficient personnel to do the work.

$\begin{array}{lllll}0 & 0 & 0 & 0 & 0\end{array}$

3. We have insufficient personnel to do the work.

$\begin{array}{lllll}0 & 0 & 0 & 0 & 0\end{array}$

4. People in the NZDF have the necessary skills to meet the NZDF's commitments.

$\begin{array}{lllll}0 & 0 & 0 & 0 & 0\end{array}$

5. The NZDF has the necessary equipment to meet its training commitments.

$\begin{array}{lllll}0 & 0 & 0 & 0 & 0\end{array}$

6. The NZDF lacks the equipment it needs to perform well in operations.

$\begin{array}{lllll}0 & 0 & 0 & 0 & 0\end{array}$

7. When I started at my workplace, an effective induction was provided

$\begin{array}{lllll}0 & 0 & 0 & 0 & 0\end{array}$

8. I am aware of the opportunities provided for voluntary education study assistance.

$\begin{array}{lllll}0 & 0 & 0 & 0 & 0\end{array}$

Which statement about Training and Resources do you consider is the most important to you?

$\begin{array}{ccccccccc}\text { None } & 1 & 2 & 3 & 4 & 5 & 6 & 7 & 8 \\ \mathrm{O} & \mathrm{O} & \mathrm{O} & \mathrm{O} & \mathrm{O} & \mathrm{O} & \mathrm{O} & \mathrm{O} & \mathrm{O}\end{array}$

\section{Section 12: Change}

1. I am well informed as to why change is occurring within my workplace.

2. I see change in the NZDF as providing new opportunities for me.

$\begin{array}{lllll}0 & 0 & 0 & 0 & 0\end{array}$

3. Changes within the last 12 months are likely to produce an NZDF that is more effective as an operational force.

$\begin{array}{lllll}0 & 0 & 0 & 0 & 0\end{array}$

4. Changes within the NZDF during the last 12 months have had a negative impact on my attitude towards future service.

5. The rate of change in the NZDF is too fast.

$\begin{array}{lllll}0 & 0 & 0 & 0 & 0\end{array}$

6. There doesn't seem to be any long term planning in the NZDF.

$\begin{array}{lllllllllll}0 & 0 & 0 & 0 & 0\end{array}$

7. I am satisfied that changes in the NZDF occur for a reason.

$\begin{array}{lllll}0 & 0 & 0 & 0 & 0\end{array}$

8. I am satisfied with the information that has been communicated to me when change occurs in my Service/the NZDF.

$\begin{array}{lllll}0 & 0 & 0 & 0 & 0\end{array}$

$\begin{array}{lllll}0 & 0 & 0 & 0 & 0\end{array}$

9. In the NZDF there is enough consultation about change with the people who will be affected by it.

10. I am sufficiently involved in decisions that affect the way I do my job.

0 O 00000

0.0000

$\begin{array}{lllll}0 & 0 & 0 & 0 & 0\end{array}$

Which statement about Change do you consider is the most important to you?

$\begin{array}{ccccccccccc}\text { None } & 1 & 2 & 3 & 4 & 5 & 6 & 7 & 8 & 9 & 10 \\ \mathrm{O} & \mathrm{O} & \mathrm{O} & \mathrm{O} & \mathrm{O} & \mathrm{O} & \mathrm{O} & \mathrm{O} & \mathrm{O} & \mathrm{O} & \mathrm{O}\end{array}$




\section{Section 13: Career Intentions}

1. It would take very little change in my present circumstances to cause me to leave the NZDF.

2. Within the past year I have considered leaving the NZDF.

000000

3. I will probably leave the NZDF in the next 12 months.

000000

4. Were I to leave the NZDF I have good employment prospects.

$\begin{array}{lllll}0 & 0 & 0 & 0 & 0\end{array}$

5. There are currently better than usual opportunities for alternative employment for me.

$\begin{array}{lllll}0 & 0 & 0 & 0 & 0\end{array}$

6. I stay because I don't think I would find as good a job outside the NZDF.

$\begin{array}{lllll}0 & 0 & 0 & 0 & 0\end{array}$

7. I would leave the NZDF if I were given a posting to somewhere that I didn't want to go.

$\begin{array}{lllll}0 & 0 & 0 & 0 & 0\end{array}$

8. I am actively looking at leaving the NZDF.

$\begin{array}{lllll}0 & 0 & 0 & 0 & 0\end{array}$

9. I am interested in joining the Reserve Forces on leaving the NZDF.

Which statement about Career Intentions do you consider is the most important to you?

$\begin{array}{cccccccccc}\text { None } & 1 & 2 & 3 & 4 & 5 & 6 & 7 & 8 & 9 \\ \mathrm{O} & \mathrm{O} & \mathrm{O} & \mathrm{O} & \mathrm{O} & \mathrm{O} & \mathrm{O} & \mathrm{O} & \mathrm{O} & \mathrm{O}\end{array}$

\section{Section 14: Morale and Satisfaction}

1. How would you rate your personal level of morale?

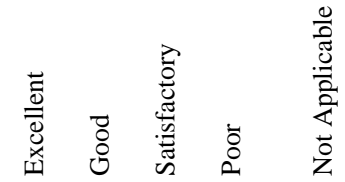

2. How would you rate the current level of morale in your workplace/section?

$\begin{array}{llll}0 & 0 & 0 & 0\end{array}$

$\begin{array}{llll}0 & 0 & 0 & 0\end{array}$

3. Considering everything how would you rate your satisfaction in your current job?

$\begin{array}{llll}0 & 0 & 0 & 0\end{array}$

4. Considering everything how would you rate your overall satisfaction with your employment in the NZDF?

$\begin{array}{llll}0 & 0 & 0 & 0\end{array}$

5. How would you rate your satisfaction with the following conditions of service/employment?

Pay

Leave

Education

Allowances

Superannuation

Family friendly initiatives

Medallic recognition

Accommodation assistance

Medical and dental

Resettlement $\begin{array}{llll}0 & 0 & 0 & 0\end{array}$

$\begin{array}{llll}0 & 0 & 0 & 0\end{array}$

$\begin{array}{llll}0 & 0 & 0 & 0\end{array}$

$\begin{array}{llll}0 & 0 & 0 & 0\end{array}$

$\begin{array}{llll}0 & 0 & 0 & 0\end{array}$

$\begin{array}{llll}0 & 0 & 0 & 0\end{array}$

$\begin{array}{lllll}0 & 0 & 0 & 0 & 0\end{array}$

$\begin{array}{lllll}0 & 0 & 0 & 0 & 0\end{array}$

$\begin{array}{lllll}0 & 0 & 0 & 0 & 0\end{array}$

$\begin{array}{lllll}0 & 0 & 0 & 0 & 0\end{array}$ 
a)

6. Overall, how satisfied are you with the military way of life?

$\begin{array}{lllll}0 & 0 & 0 & 0 & 0\end{array}$

\section{Section 15: General}

1. Have you returned from an Operational Deployment (Output Class 16) within the past 12 months? Yes $\mathrm{O}$

No $\mathrm{O}$

2. If you have returned from an Operational Deployment (Output Class 16) within the past 12 months, how long were you deployed for?

$\begin{array}{ll}\text { N/A } & \text { O } \\ \text { Up to } 3 \text { months } & \text { O } \\ 4 \text { to } 6 \text { months } & \text { O } \\ 7 \text { to } 9 \text { months } & \text { O } \\ 10 \text { to } 12 \text { months } & \text { O } \\ \text { More than } 12 \text { months } & \text { O }\end{array}$

3. How many Operational Deployments (Output Class 16) have you been on?

$\begin{array}{ll}\text { None } & \text { O } \\ \text { One } & \text { O } \\ \text { Two } & \text { O } \\ \text { Three } & \text { O } \\ \text { Four } & 0 \\ \text { Five or more } & 0\end{array}$

4. How much time have you spent away from your home location, on any tour of duty in the last 12 months (including training, exercises, and any deployments)?

None

0

Less than two weeks

0

Between two weeks and one month $\mathrm{O}$

Between one and three months

O

Between three and six months

O

More than six months

O

5. When you joined the NZDF, how long did you intend to stay for? Please select one.

$\begin{array}{ll}\text { Less than } 5 \text { years } & 0 \\ 6 \text { to } 10 \text { years } & 0 \\ 11 \text { to } 15 \text { years } & 0\end{array}$


16 to 20 years

6. For these two questions, select one reason from the following list and place the number of the item into the box beside each question.

a. What was your main reason for joining the NZDF? (write number from list below)

If 12 (other), please specify reason for joining:

b. What is your main reason for staying in the NZDF? (write number from list below)

If 12 (other), please specify reason for staying:

1. Travel

2. Friendship

3. Valuable skills/training

4. Challenging and interesting work

5. Physical Fitness

6. Job security

7. Leadership roles

8. Adventure

9. Patriotic duty

10. Family/work balance

11. Job satisfaction

12. Other

7. What would be your main reason for leaving the NZDF? Please select one.

Education

$\mathrm{O}$

Travel

O

Internal employment opportunities

External employment opportunities

Job satisfaction

Family/work balance

NZDF Leadership

Completed service

Personal - within NZDF

O

Personal - outside NZDF

O

No reason to leave

O

Other (please specify):

O

8. From the following list, what three aspects of your employment/engagement do you feel most require change? Place the numbers of the items in the boxes, with "One” being the highest priority for change.

1. Conditions I work in

2. Amount I am paid 
3. Amount of work I am expected to do

One

4. Training and / or development opportunities

5. Unsatisfactory work equipment

Two

6. Job security

7. Recognition I get for doing my job

Three

8. Performance feedback

9. Promotion opportunities

10. Communication about change

11. Relocation/posting cycle

12. Other (please specify)

9. From the following list, what three aspects of the NZDF do you feel most require change? Place the numbers of the items in the boxes, with "One" being the highest priority for change.

1. Level of job security

2. Retaining personnel

One

3. Communication about change

4. Consultation e.g. when change is introduced

Two

5. Equipment

6. Military capability

Three

7. Profile of NZDF in society

8. Commitment from senior leaders

9. Other (please specify)

Do you wish to make any further comments? 
Please ensure you have filled in the questionnaire as directed on page 2

Please now return the questionnaire in the self-addressed envelope provided.

Please do not fold the questionnaire, leave it A4 size and do not staple the questionnaire together.

You are welcome to look at the latest results from the survey under Psychology on the Personnel

Branch intranet site @ http://ilp/FrameTool.asp?Target=http://dcsbhost2/pers/persbranch.htm

\section{Thank-you once again for your time and effort in filling out this \\ survey.}

